\title{
Chiral vicinal diamines derived from Mefloquine
}

Dawid J. Kucharski, ${ }^{a}$ Rafał Kowalczyk, ${ }^{b}$ and Przemysław J. Boratyński ${ }^{\text {a* }}$

${ }^{a}$ Department of Organic and Medicinal Chemistry, Wroctaw University of Technology, Wyb. Wyspiańskiego 26, Wrocław, 50-370 Poland

${ }^{b}$ Department of Bioorganic chemistry, Wrockaw University of Technology,

Wyb. Wyspiańskiego 26, Wrocław, 50-370 Poland

*e-mail: przemyslaw.boratynski@pwr.edu.p1

$\underline{\text { Table of contents }}$

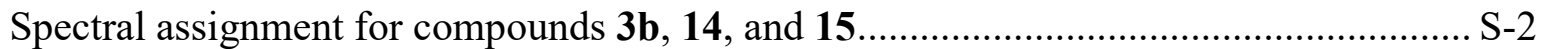

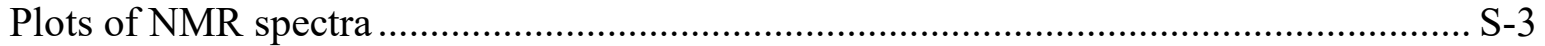

Spectral data for 11-chloro-13-benzyl-mefloquine hydrochloride................................. S-41

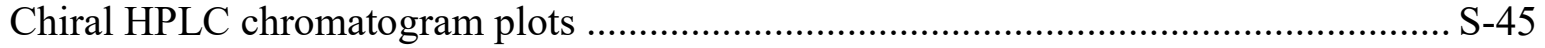

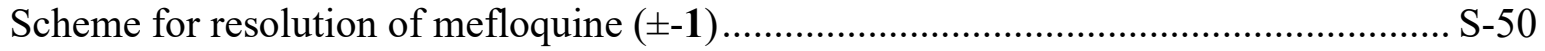

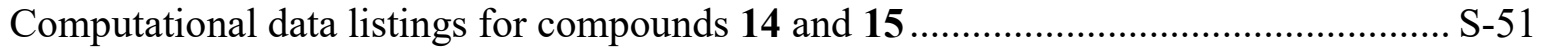




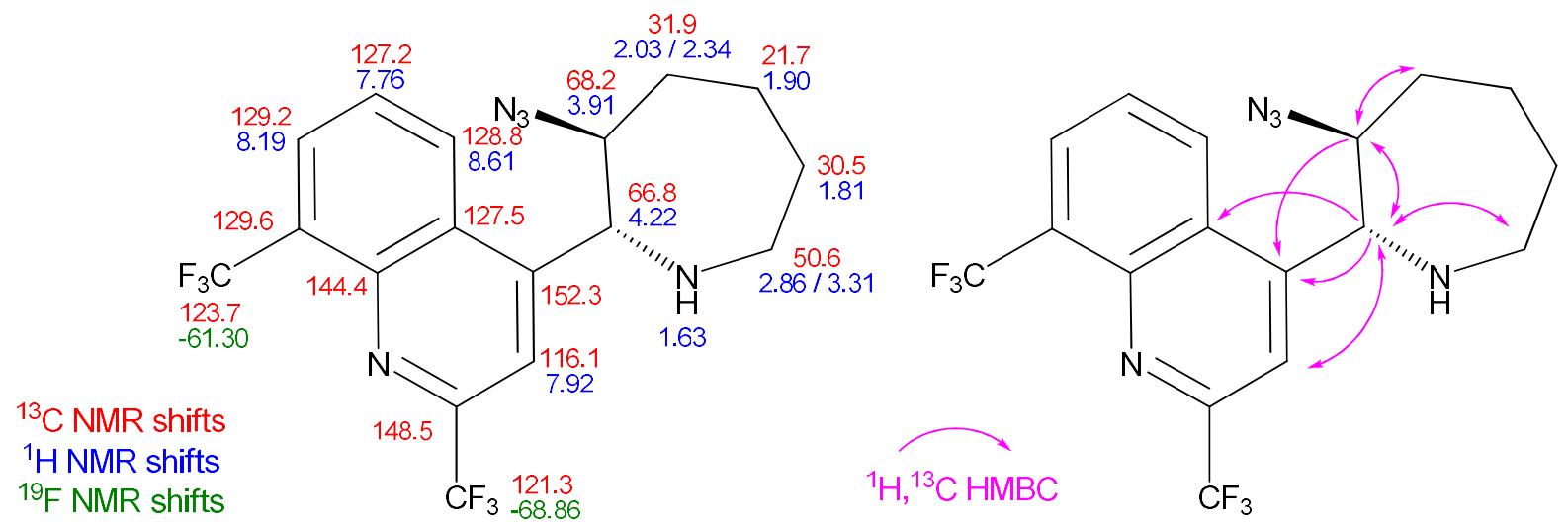

Figure S1. Spectral assignment for $\mathbf{3 b}$ and diagnostic ${ }^{1} \mathrm{H},{ }^{13} \mathrm{C}-\mathrm{HMBC}$ interactions for aliphatic methine $(\mathrm{CH})$ groups.
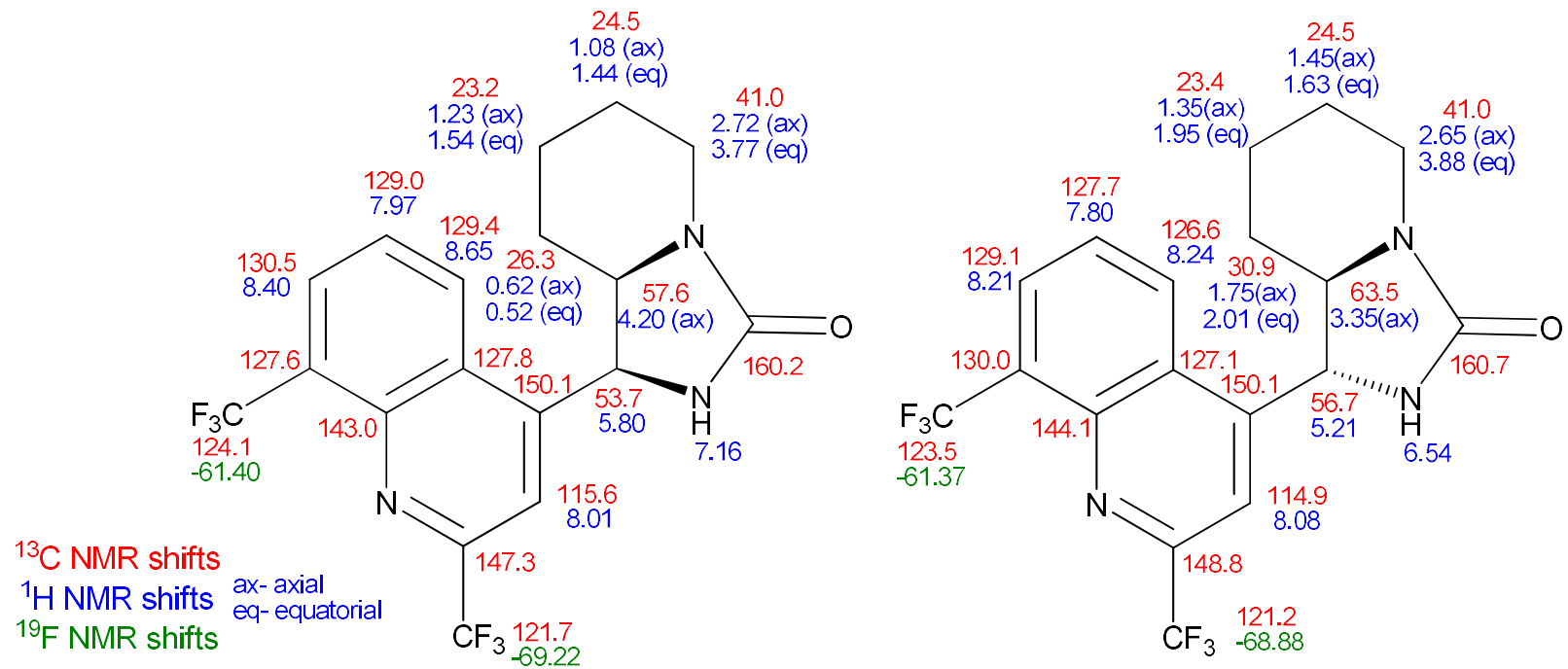

Figure S2 Spectral assignment for cyclic urea derived from erythro-11-aminomefloquine (14, left) and threo-11-aminomefloquine (15, right). 

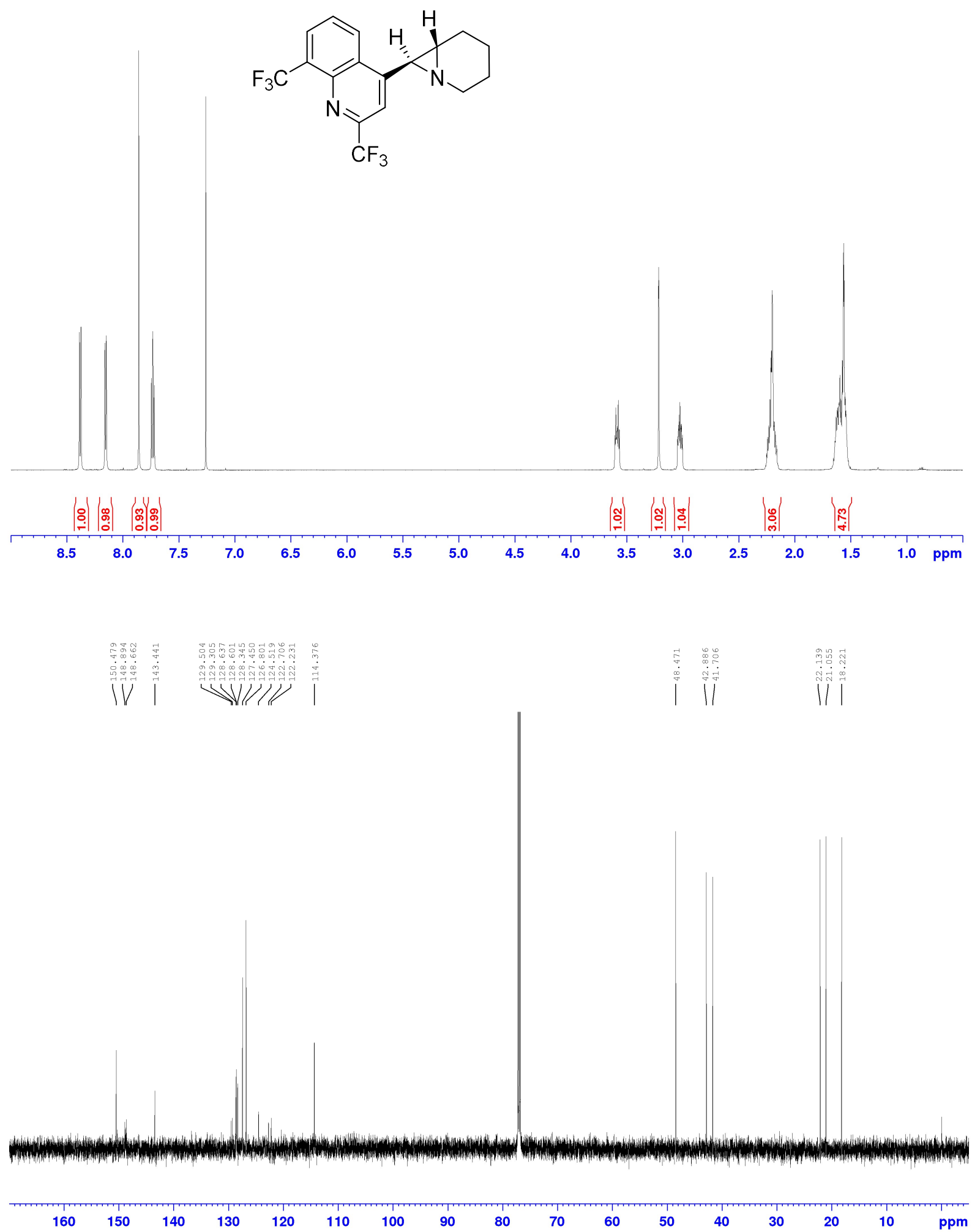

Figure S3. ${ }^{1} \mathrm{H}(600 \mathrm{MHz})$ and ${ }^{13} \mathrm{C}\left\{{ }^{1} \mathrm{H}\right\}$ NMR $(151 \mathrm{MHz})$ spectra for 2 in $\mathrm{CDCl}_{3}+\mathrm{TMS}$ 

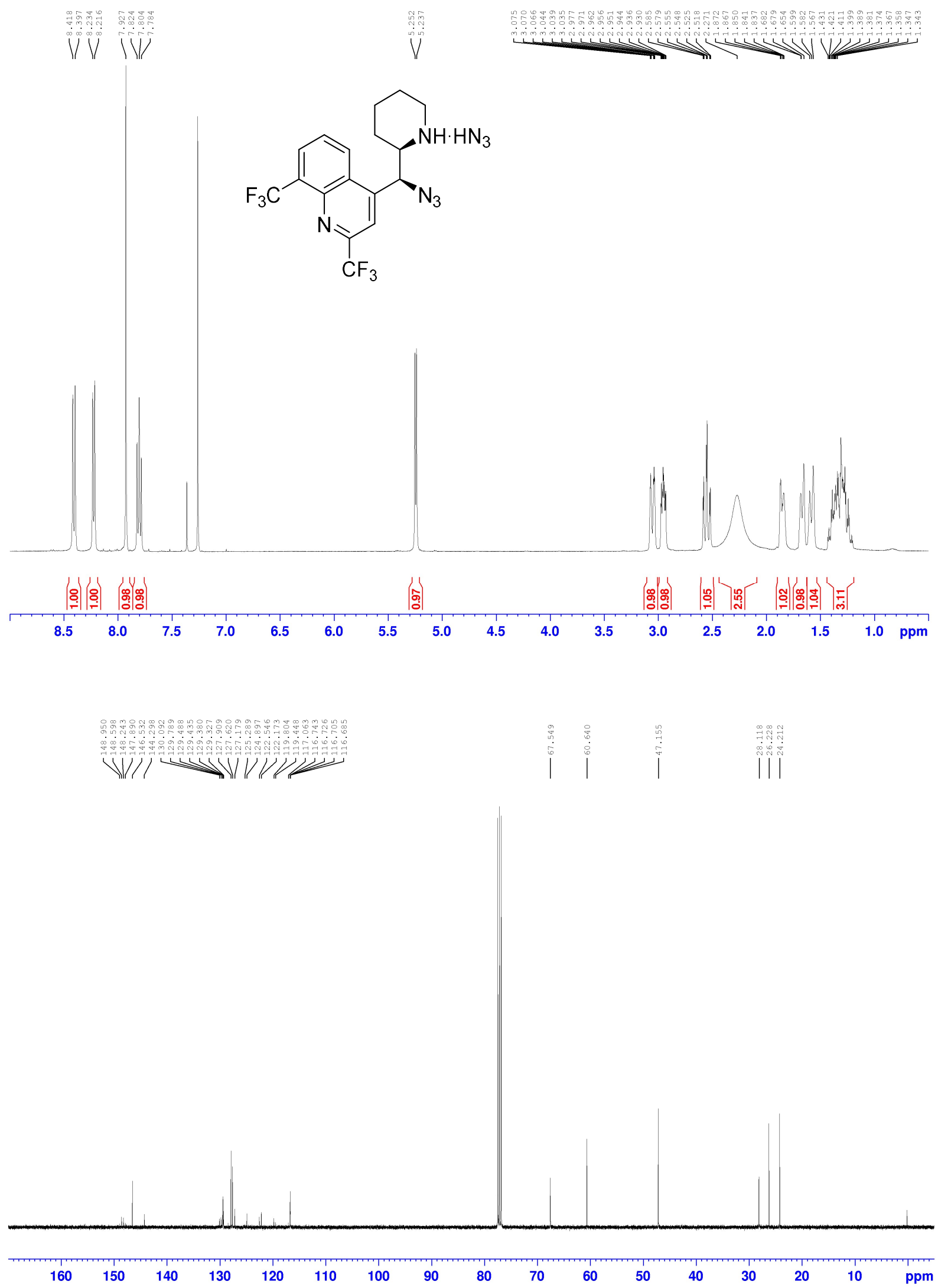

Figure S4. ${ }^{1} \mathrm{H}(400 \mathrm{MHz})$ and ${ }^{13} \mathrm{C}\left\{{ }^{1} \mathrm{H}\right\} \mathrm{NMR}(100 \mathrm{MHz})$ spectra for $\mathbf{3} \cdot \mathrm{HN}_{3}$ in $\mathrm{CDCl}_{3}+\mathrm{TMS}$ 
<smiles>NC(c1cc(C(F)(F)F)nc2c(C(F)(F)F)cccc12)C1CCCCN1</smiles>

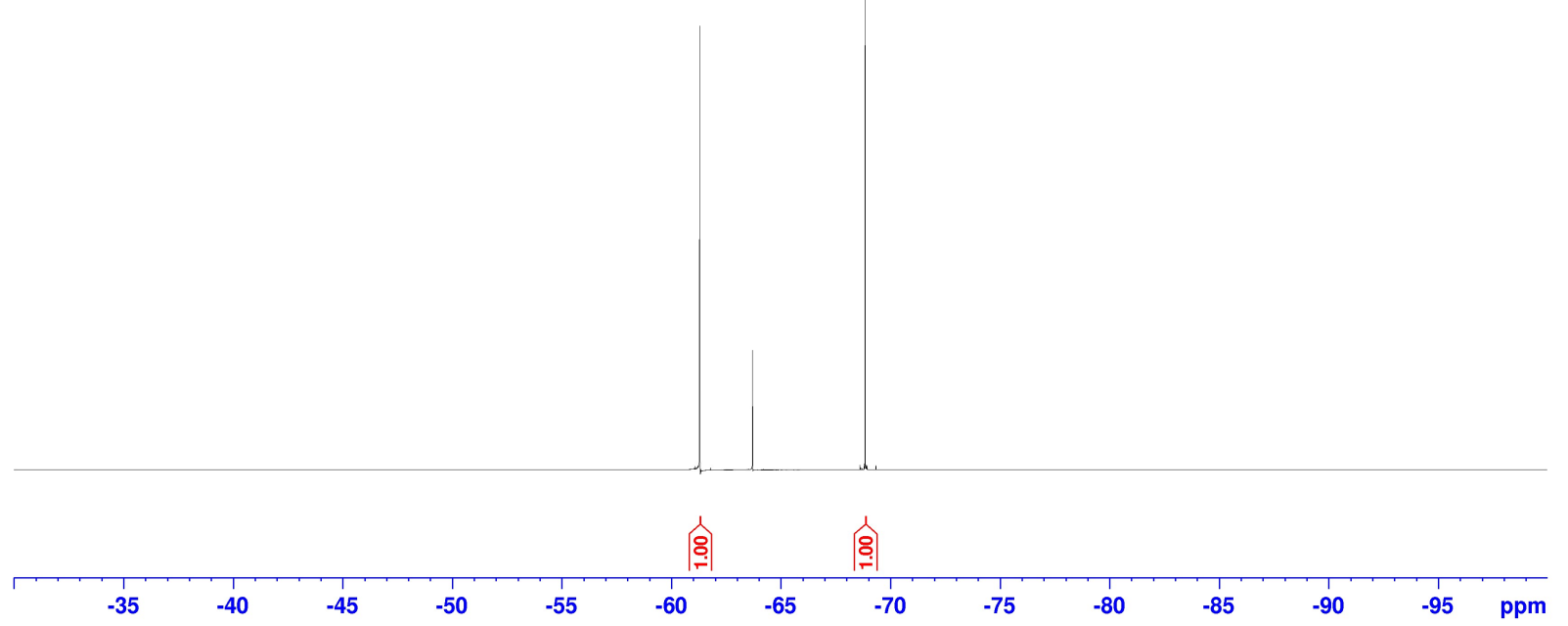

Figure S5. ${ }^{19} \mathrm{~F}$ NMR $(376 \mathrm{MHz})$ spectrum for $3 \cdot \mathrm{HN}_{3}$ in $\mathrm{CDCl}_{3}+\mathrm{PhCF}_{3}\left(\delta_{\mathrm{F}}=-63.72 \mathrm{ppm}\right)$ 

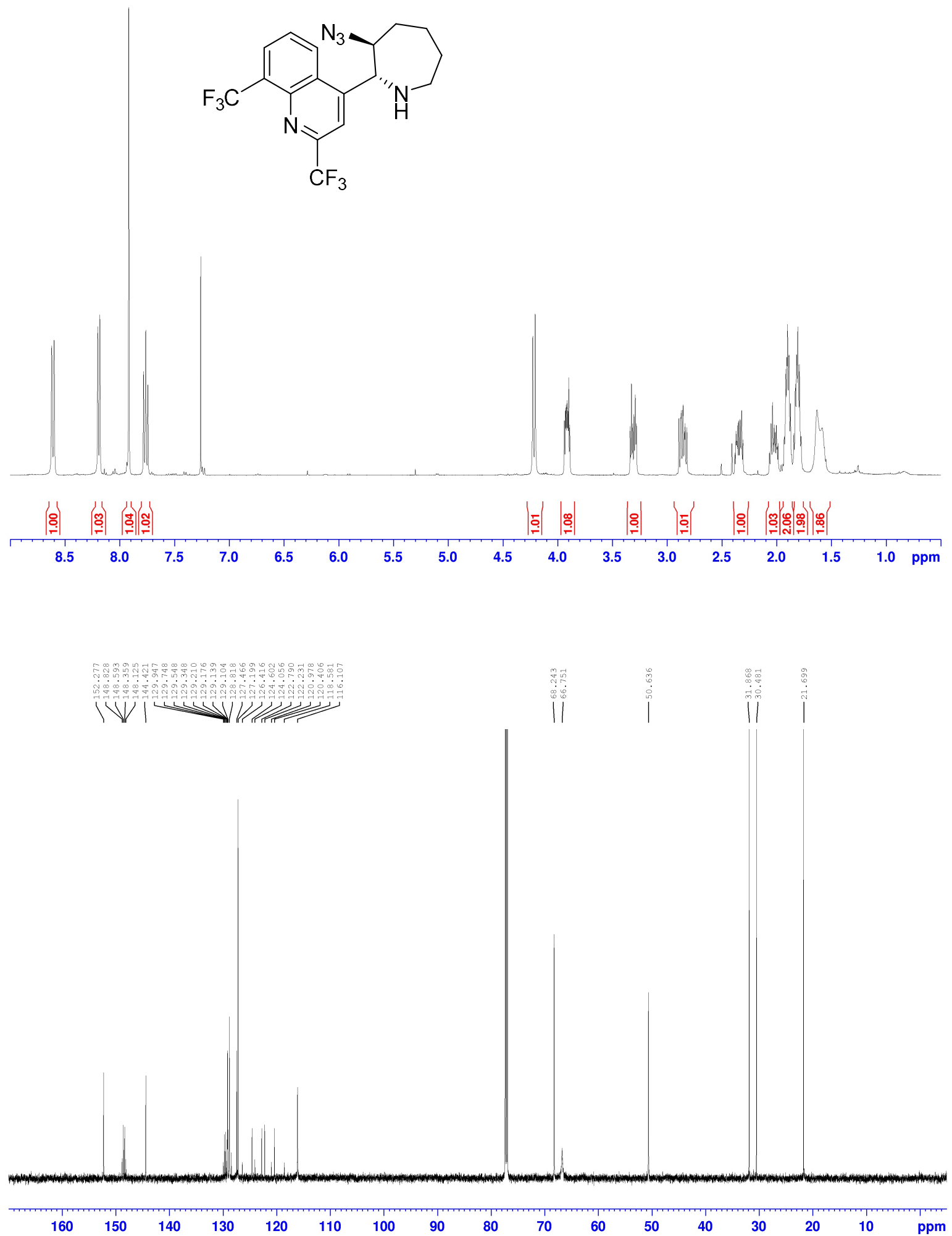

Figure S6. ${ }^{1} \mathrm{H}(400 \mathrm{MHz})$ and ${ }^{13} \mathrm{C}\left\{{ }^{1} \mathrm{H}\right\} \mathrm{NMR}(151 \mathrm{MHz})$ spectra for $\mathbf{3 b}$ in $\mathrm{CDCl}_{3}+\mathrm{TMS}$ 


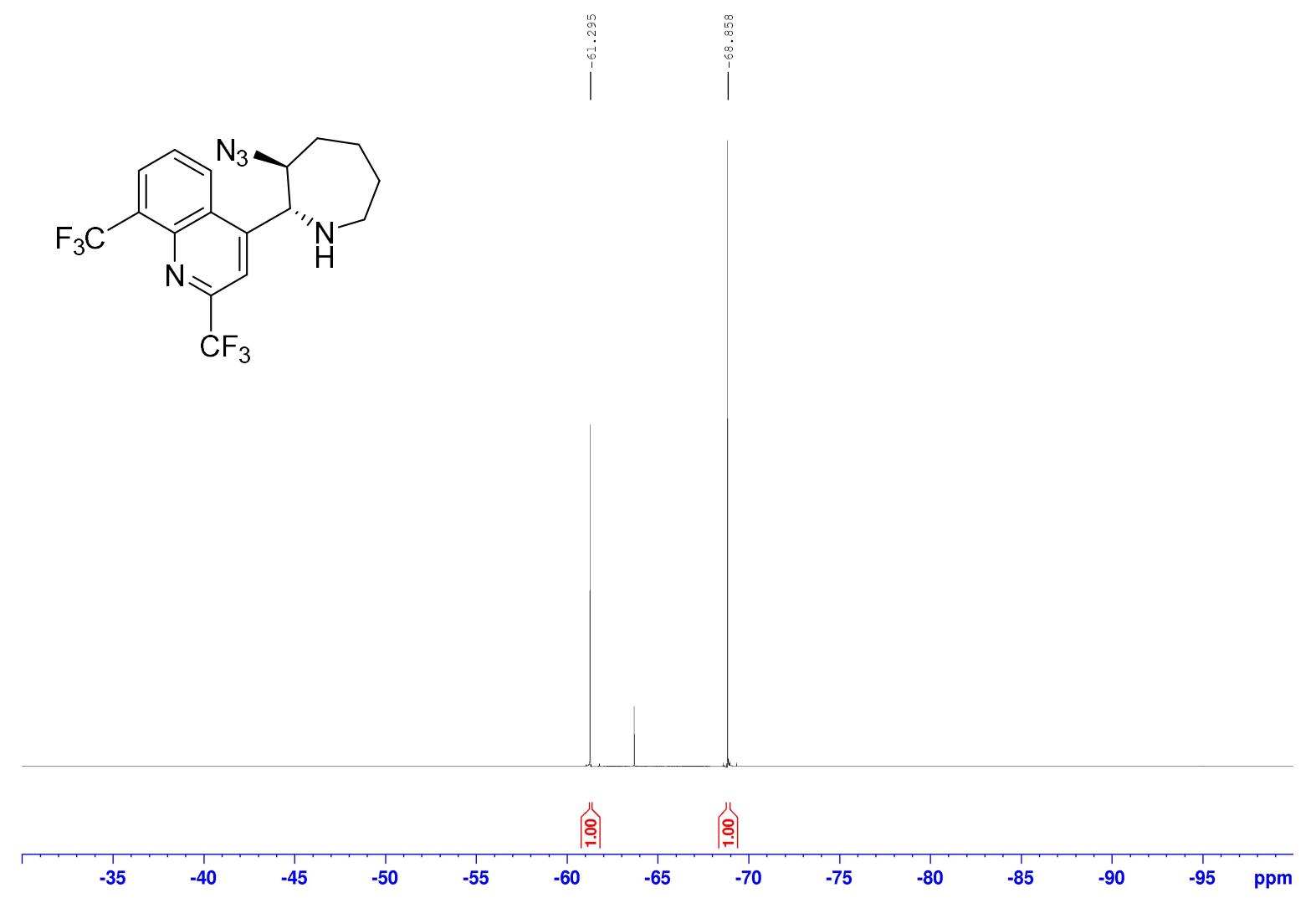

Figure S7. ${ }^{19} \mathrm{~F}$ NMR $(376 \mathrm{MHz})$ spectrum for $\mathbf{3 b}$ in $\mathrm{CDCl}_{3}+\mathrm{PhCF}_{3}\left(\delta_{\mathrm{F}}=-63.72 \mathrm{ppm}\right)$

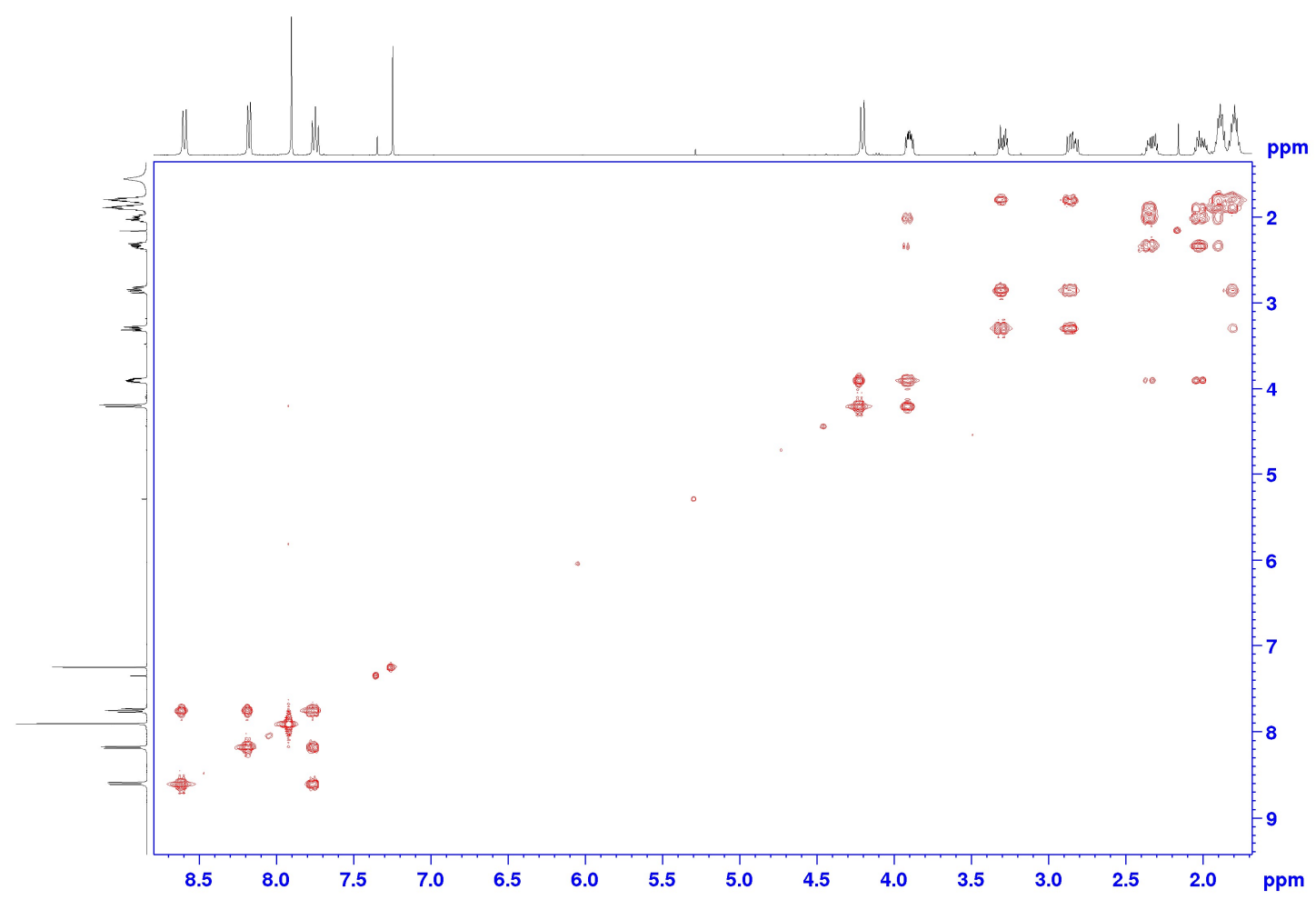

Figure S8. ${ }^{1} \mathrm{H}$ COSY experiment $(600 \mathrm{MHz})$ for $\mathbf{3 b}$ in $\mathrm{CDCl}_{3}$. For spectral assignment, see Figure S1. 

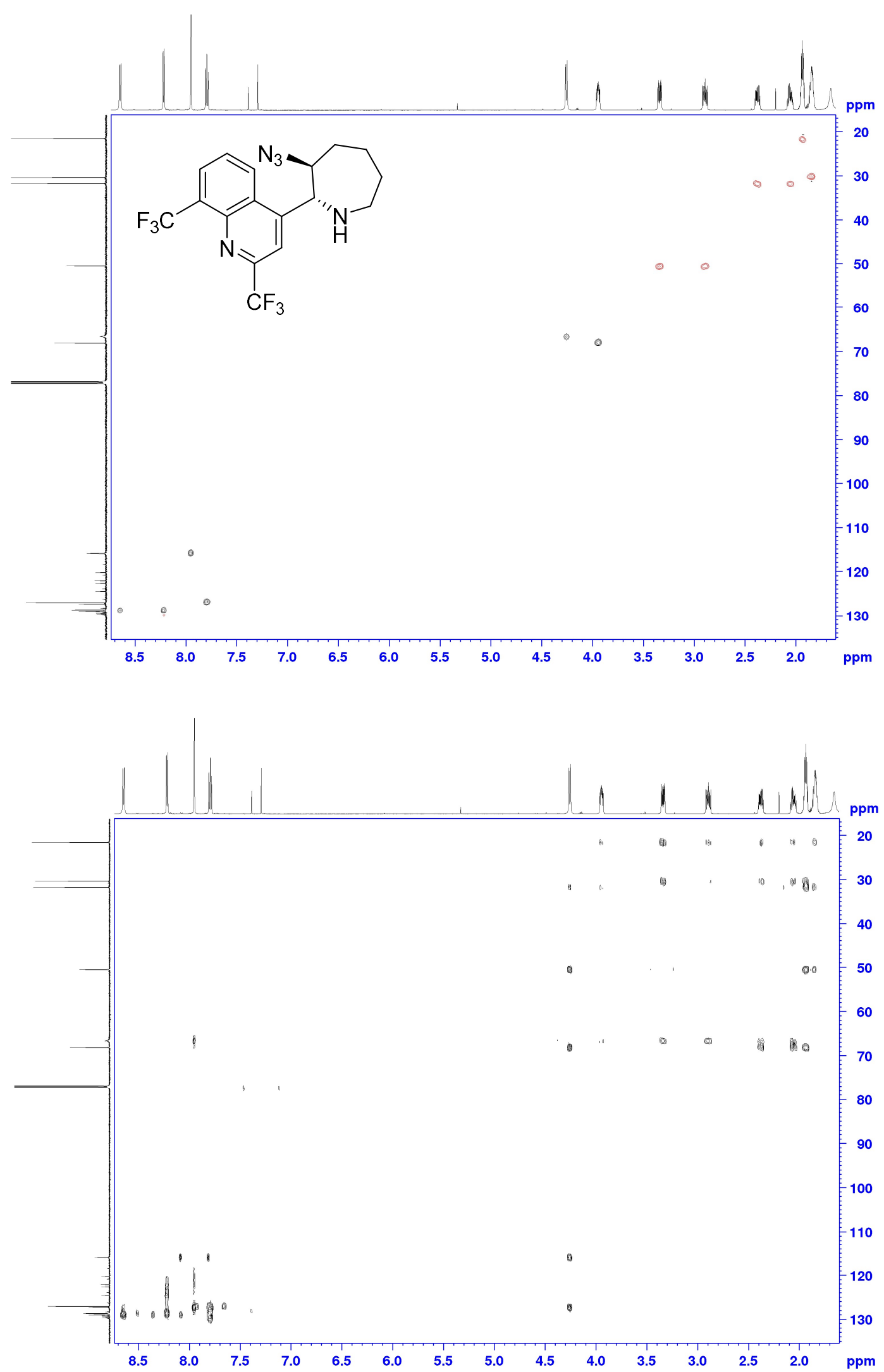

Figure S9. ${ }^{1} \mathrm{H},{ }^{13} \mathrm{C}$ multiplicity-resolved $\mathrm{HSQC}\left(\mathrm{CH}_{2}\right.$-red, $\mathrm{CH} / \mathrm{CH}_{3}$-black; top) and $\mathrm{HMBC}$ (bottom) experiments for $\mathbf{3 b}$ in $\mathrm{CDCl}_{3}$. For spectral assignment, see Figure $\mathrm{S} 1$. 

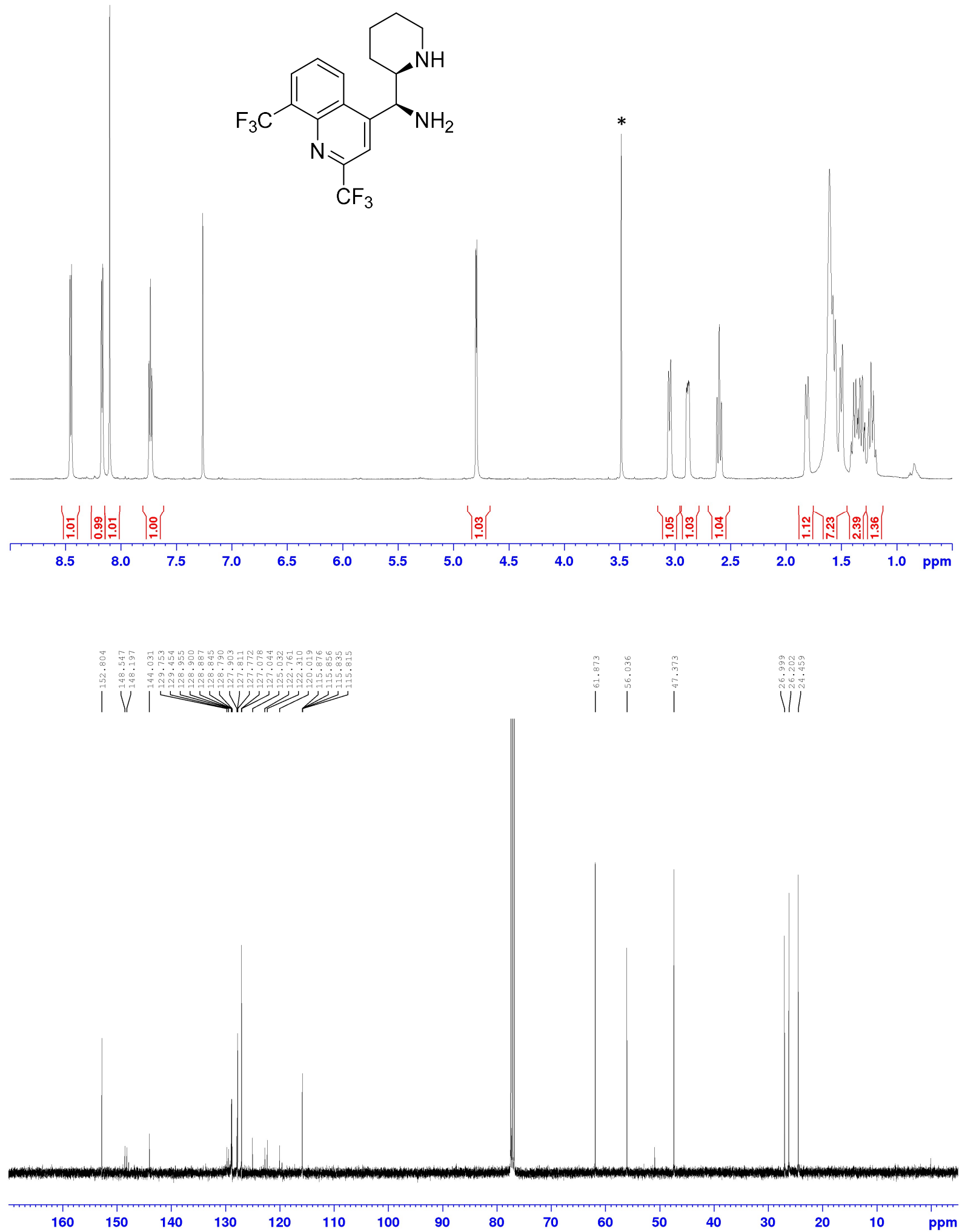

Figure S10. ${ }^{1} \mathrm{H}(400 \mathrm{MHz})$ and ${ }^{13} \mathrm{C}\left\{{ }^{1} \mathrm{H}\right\} \mathrm{NMR}(100 \mathrm{MHz})$ spectra for 4 in $\mathrm{CDCl}_{3}+\mathrm{TMS}\left({ }^{*}\right.$ sample contains residue of $\mathrm{MeOH}$ from crystalline solvate) 


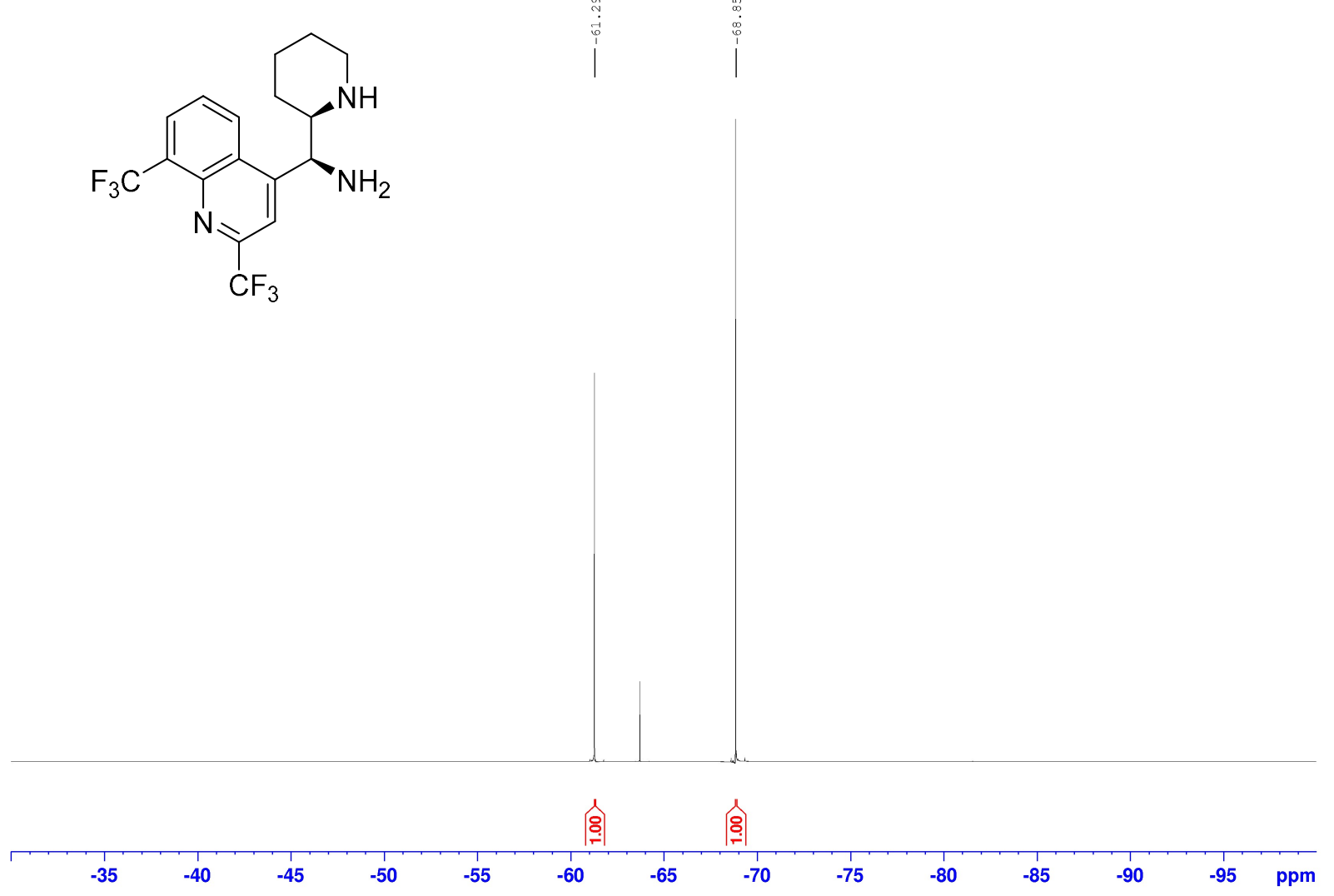

Figure S11. ${ }^{19} \mathrm{~F} \mathrm{NMR}(376 \mathrm{MHz})$ spectrum for 4 in $\mathrm{CDCl}_{3}+\mathrm{PhCF}_{3}\left(\delta_{\mathrm{F}}=-63.72 \mathrm{ppm}\right)$ 


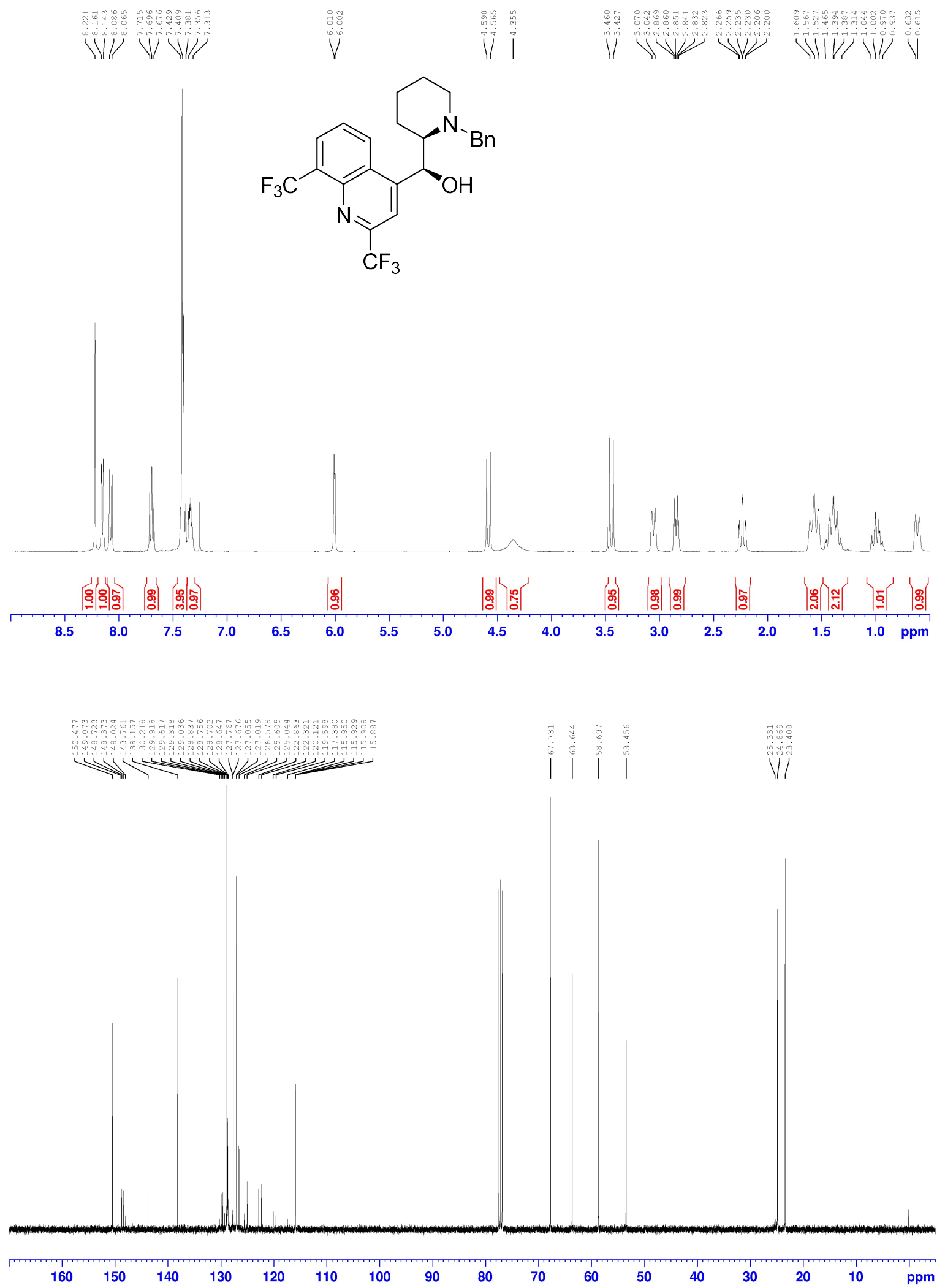

Figure S12. ${ }^{1} \mathrm{H}(400 \mathrm{MHz})$ and ${ }^{13} \mathrm{C}\left\{{ }^{1} \mathrm{H}\right\}$ NMR $(100 \mathrm{MHz})$ spectra for $\mathbf{5 a}$ in $\mathrm{CDCl}_{3}+\mathrm{TMS}$ 
<smiles>CN1CCCCC1[C@H](O)c1cc(C(F)(F)F)nc2c(C(F)(F)F)cccc12</smiles>
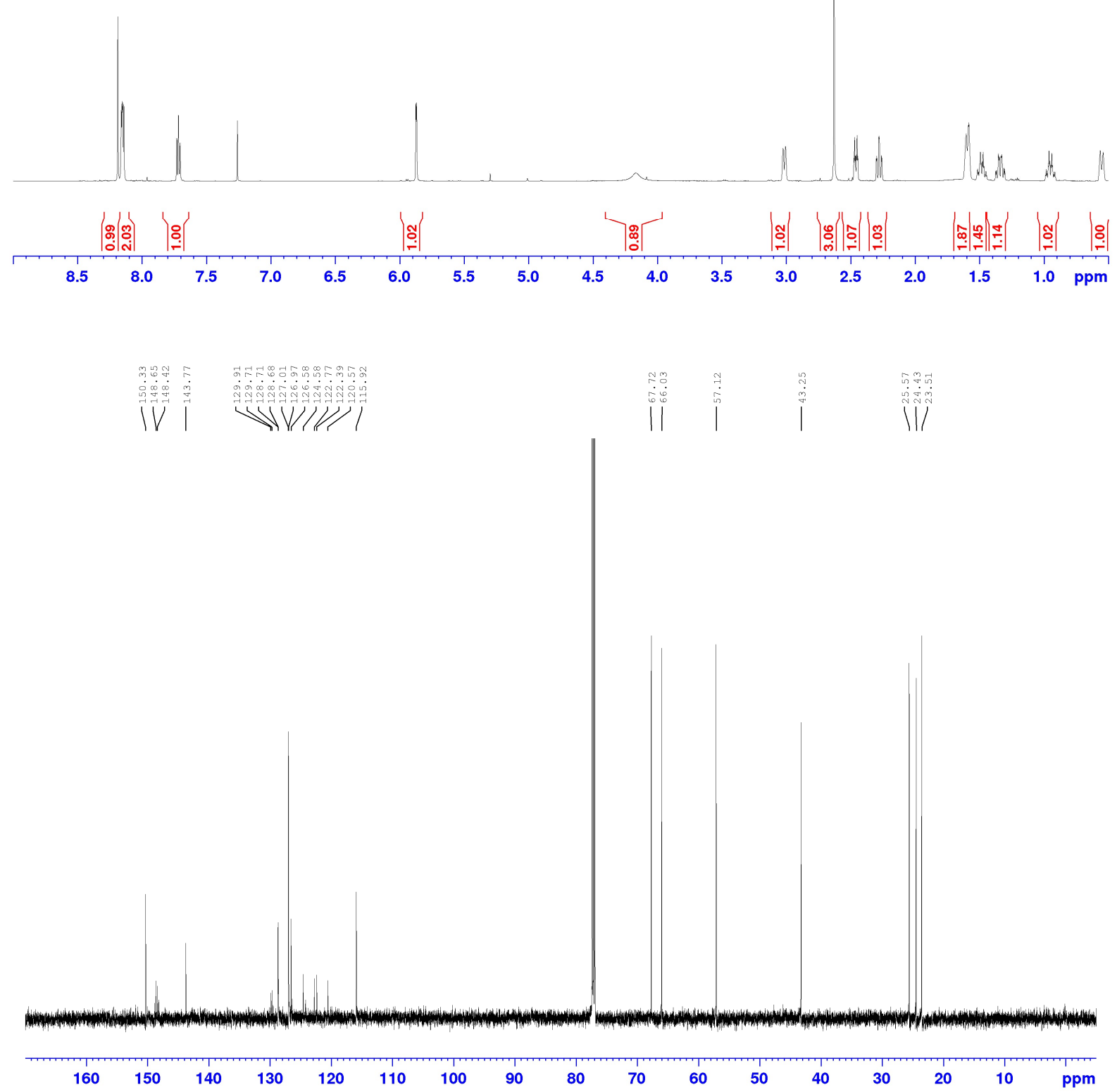

Figure S13. ${ }^{1} \mathrm{H}(600 \mathrm{MHz})$ and ${ }^{13} \mathrm{C}\left\{{ }^{1} \mathrm{H}\right\} \mathrm{NMR}(151 \mathrm{MHz})$ spectra for $\mathbf{5 b}$ in $\mathrm{CDCl}_{3}+\mathrm{TMS}$ 


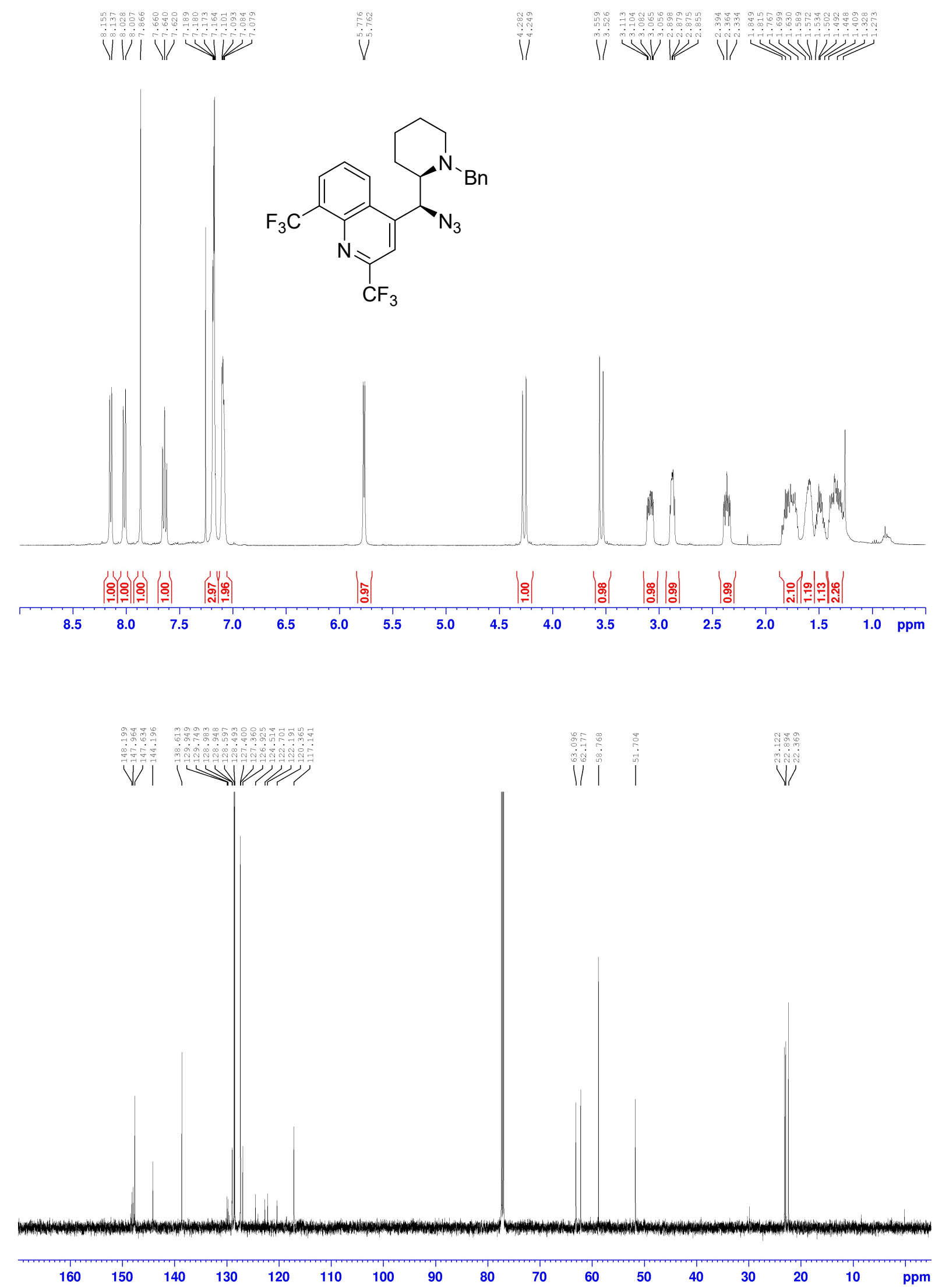

Figure S14. ${ }^{1} \mathrm{H}(600 \mathrm{MHz})$ and ${ }^{13} \mathrm{C}\left\{{ }^{1} \mathrm{H}\right\} \mathrm{NMR}(151 \mathrm{MHz})$ spectra for $\mathbf{6 a}$ in $\mathrm{CDCl}_{3}+\mathrm{TMS}$ 


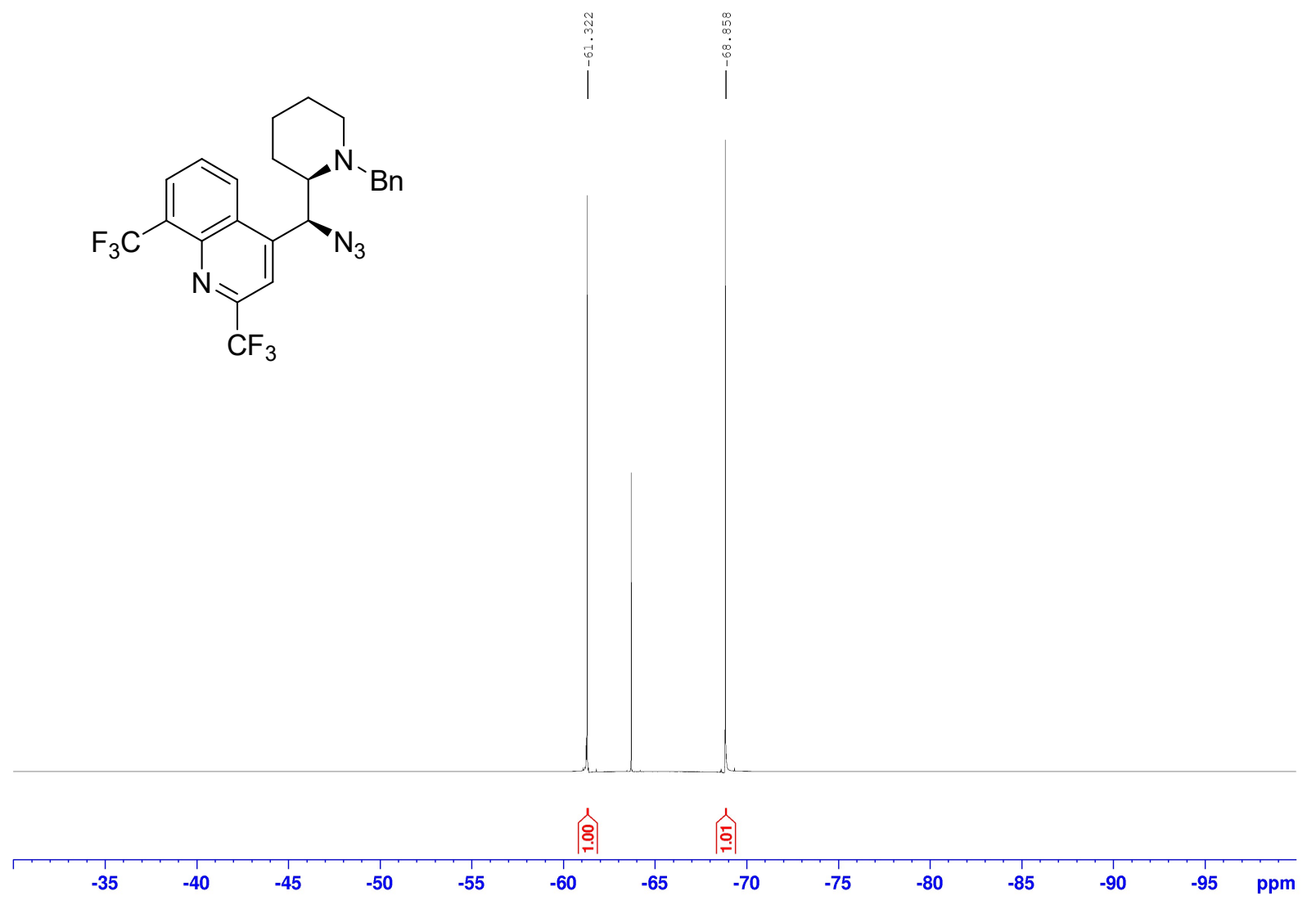

Figure S15. ${ }^{19} \mathrm{~F}$ NMR $(376 \mathrm{MHz})$ spectrum for $6 \mathbf{a}$ in $\mathrm{CDCl}_{3}+\mathrm{PhCF}_{3}\left(\delta_{\mathrm{F}}=-63.72 \mathrm{ppm}\right)$ 
<smiles>CN1CCCCC1[C@H](N)c1cc(C(F)(F)F)nc2c(C(F)(F)F)cccc12</smiles>
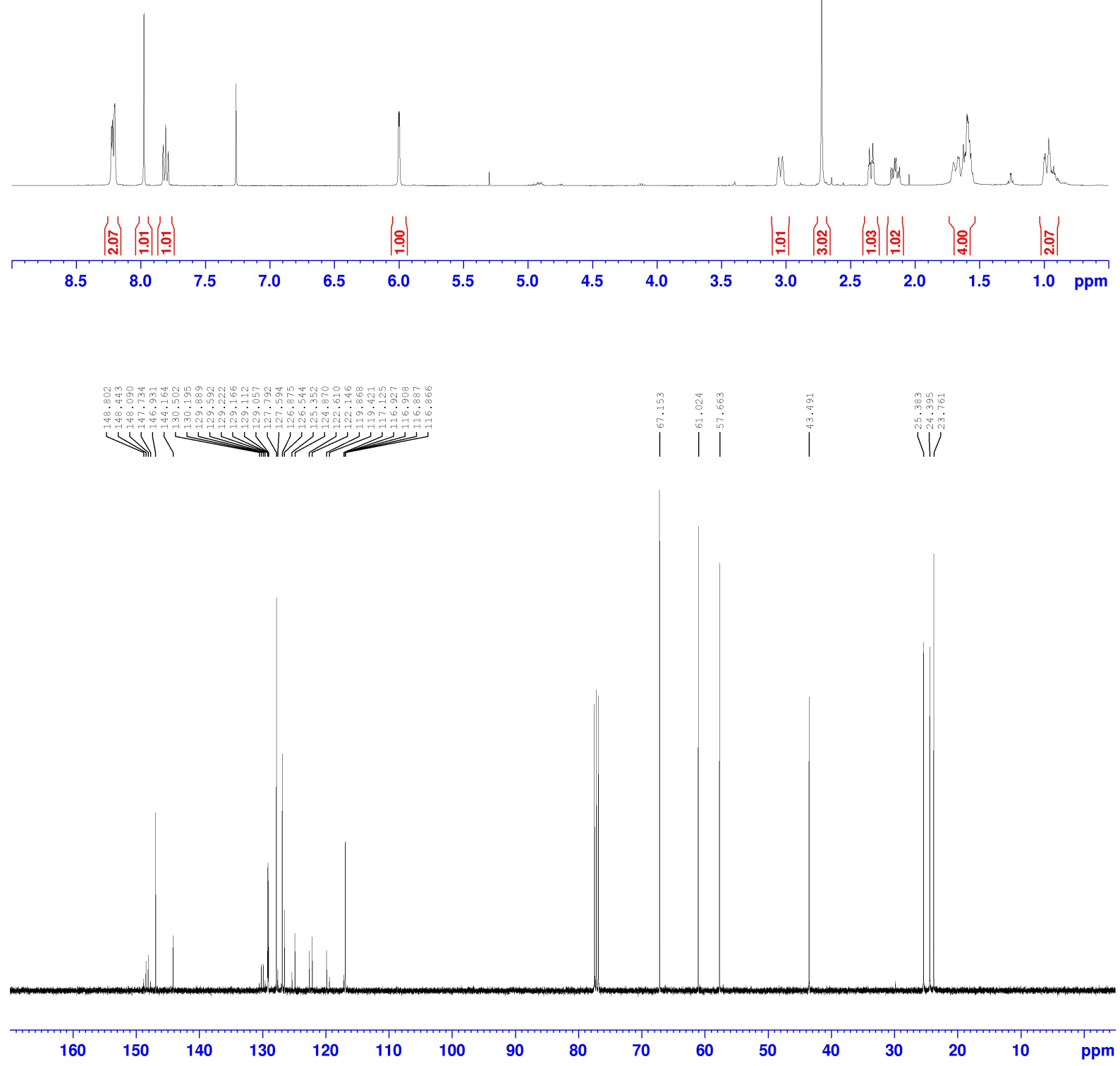

Figure S16. ${ }^{1} \mathrm{H}(400 \mathrm{MHz})$ and ${ }^{13} \mathrm{C}\left\{{ }^{1} \mathrm{H}\right\} \mathrm{NMR}(100 \mathrm{MHz})$ spectra for $\mathbf{6 b}$ in $\mathrm{CDCl}_{3}+\mathrm{TMS}$ 


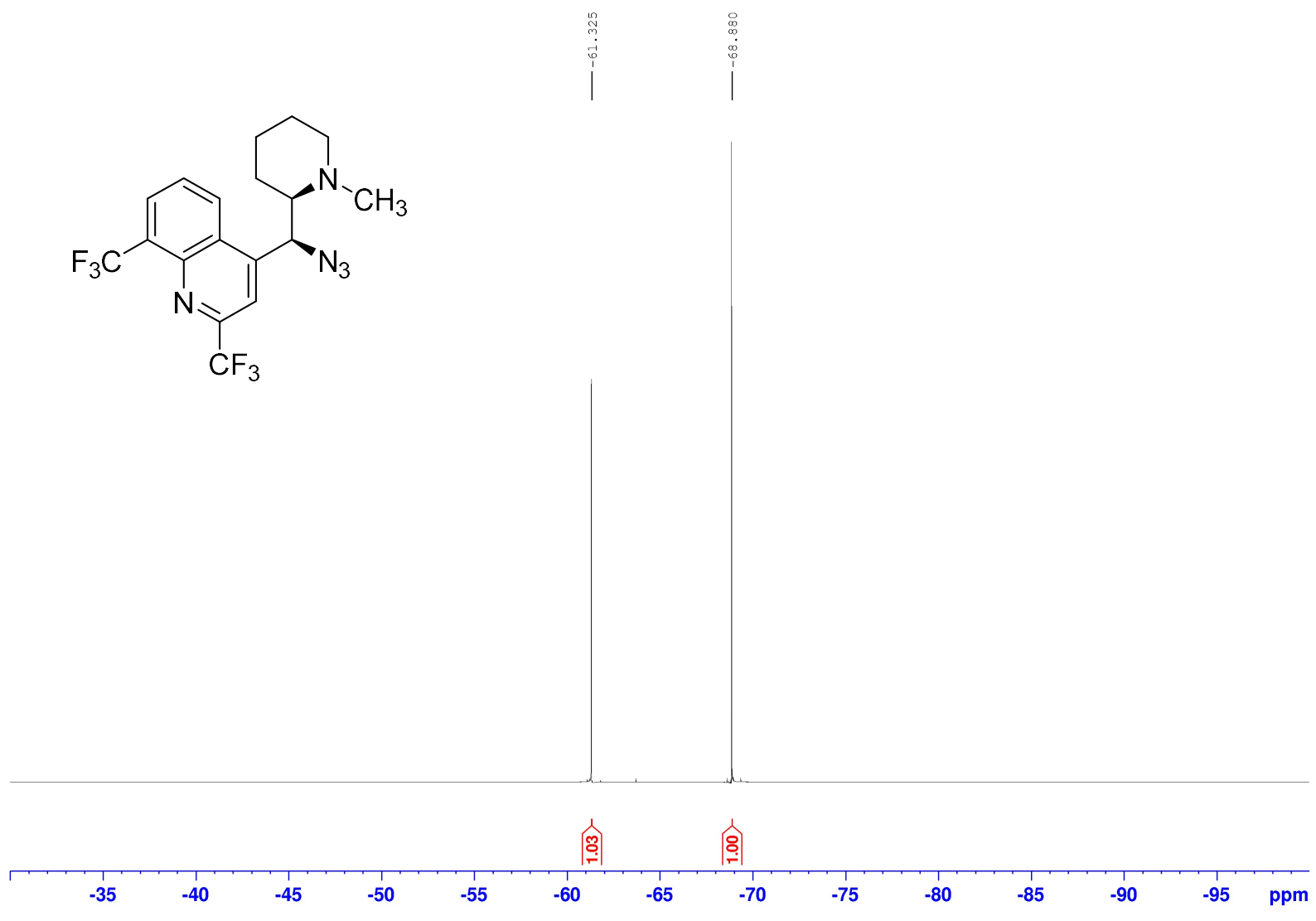

Figure S17. ${ }^{19} \mathrm{~F}$ NMR $(376 \mathrm{MHz})$ spectrum for $6 \mathbf{b}$ in $\mathrm{CDCl}_{3}+\mathrm{PhCF}_{3}\left(\delta_{\mathrm{F}}=-63.72 \mathrm{ppm}\right)$ 

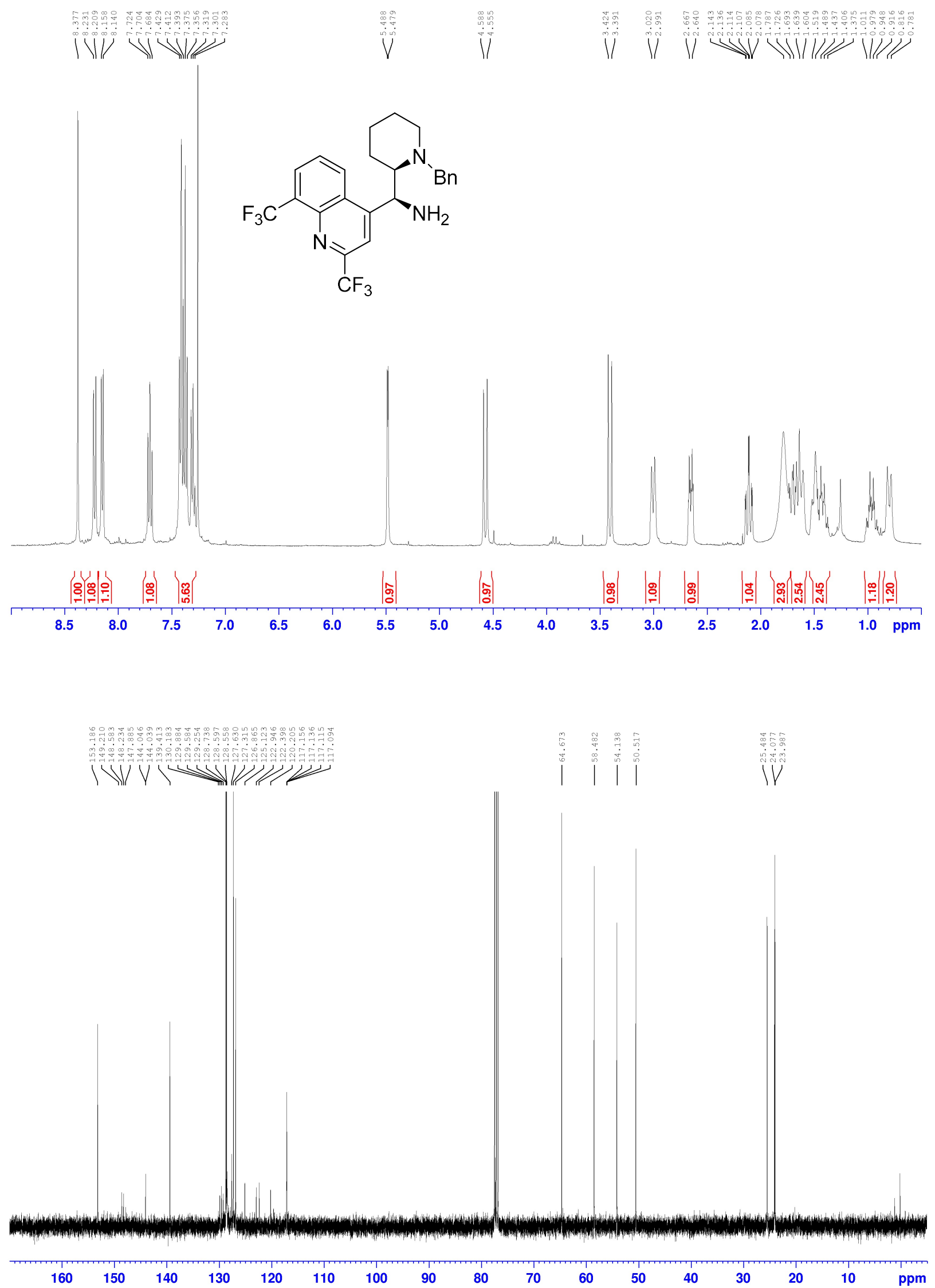

Figure S18. ${ }^{1} \mathrm{H}(400 \mathrm{MHz})$ and ${ }^{13} \mathrm{C}\left\{{ }^{1} \mathrm{H}\right\}$ NMR $(100 \mathrm{MHz})$ spectra for 7a in $\mathrm{CDCl}_{3}+\mathrm{TMS}$ 
<smiles>NC(c1cc(C(F)(F)F)nc2c(C(F)(F)F)cccc12)C1CCCCN1Cc1ccccc1</smiles>

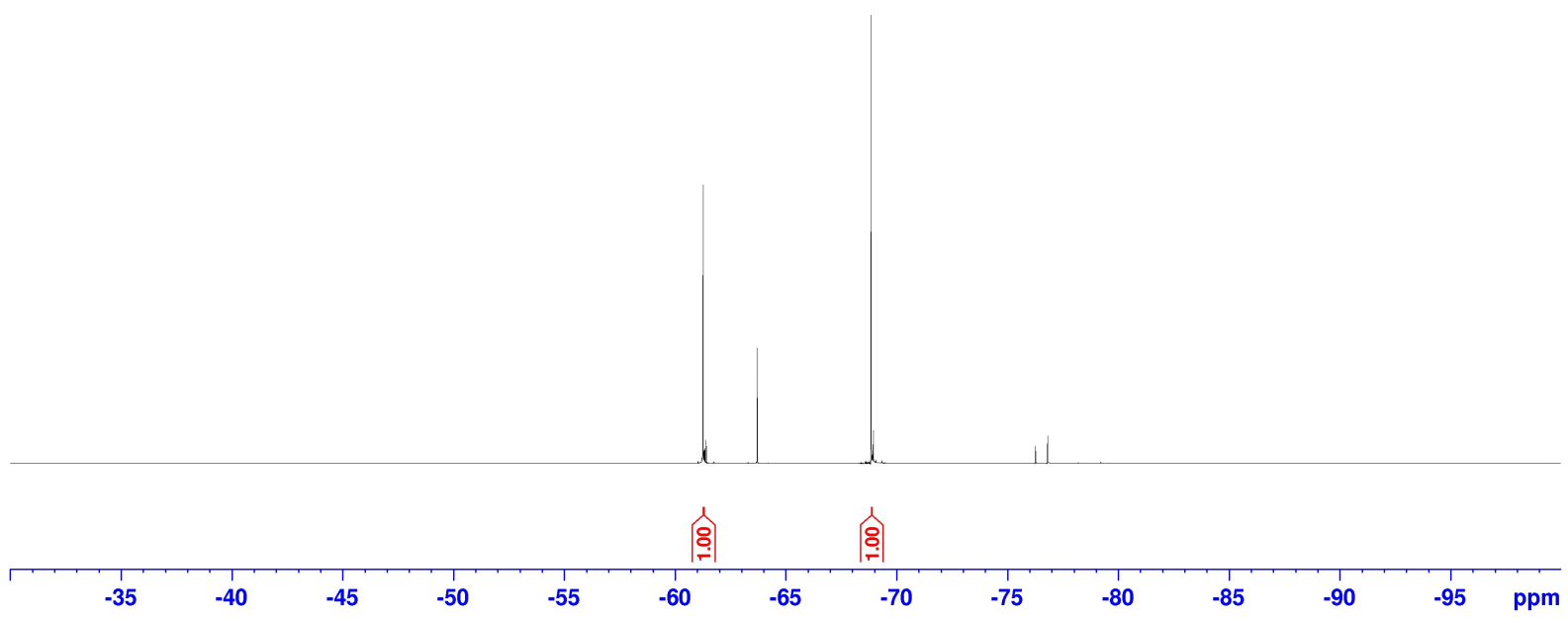

Figure S19. ${ }^{19} \mathrm{~F}$ NMR $(376 \mathrm{MHz})$ spectrum for $7 \mathbf{a}$ in $\mathrm{CDCl}_{3}+\mathrm{PhCF}_{3}\left(\delta_{\mathrm{F}}=-63.72 \mathrm{ppm}\right)$ 

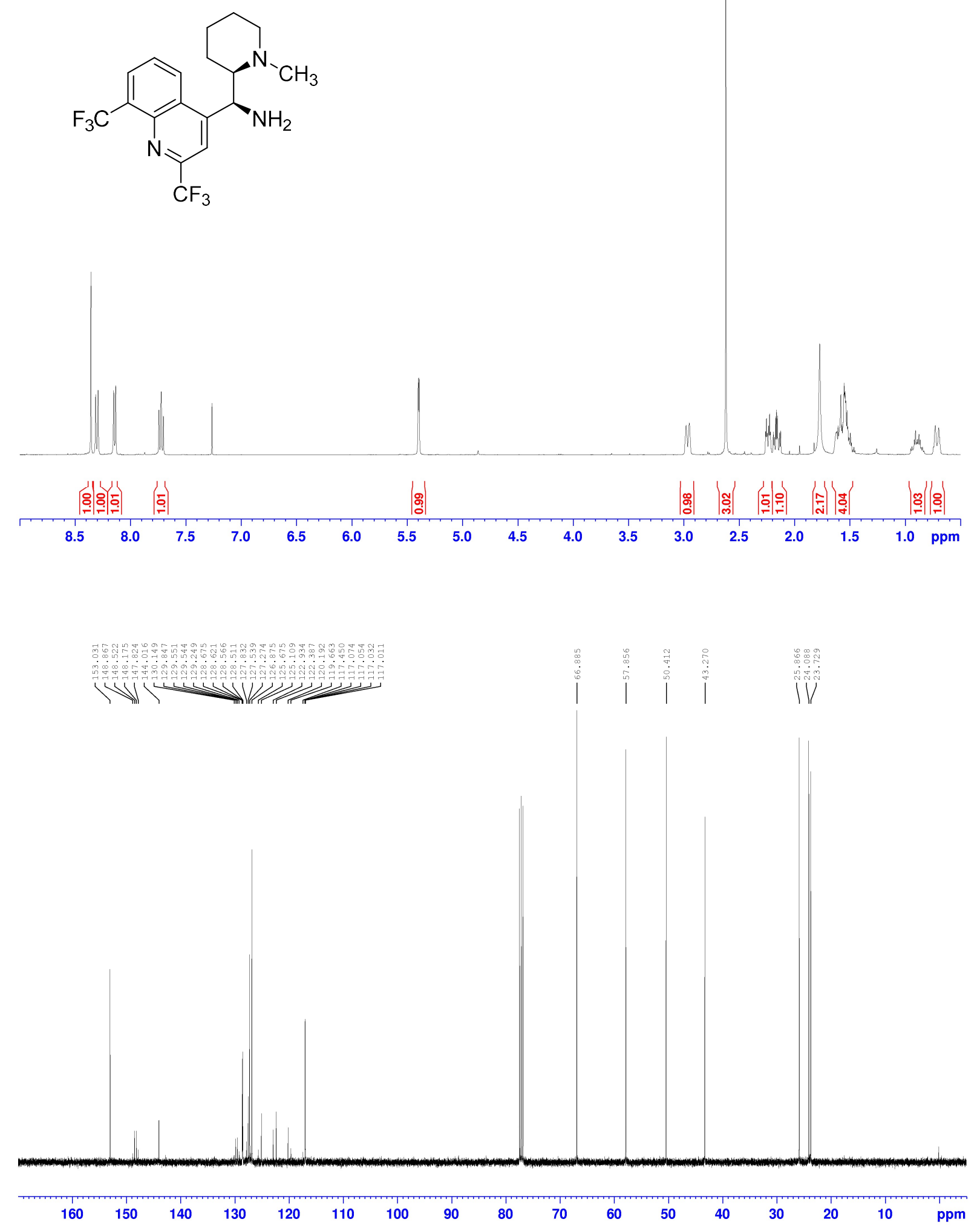

Figure S20. ${ }^{1} \mathrm{H}(400 \mathrm{MHz})$ and ${ }^{13} \mathrm{C}\left\{{ }^{1} \mathrm{H}\right\} \mathrm{NMR}(100 \mathrm{MHz})$ spectra for $7 \mathbf{b}$ in $\mathrm{CDCl}_{3}+\mathrm{TMS}$ 


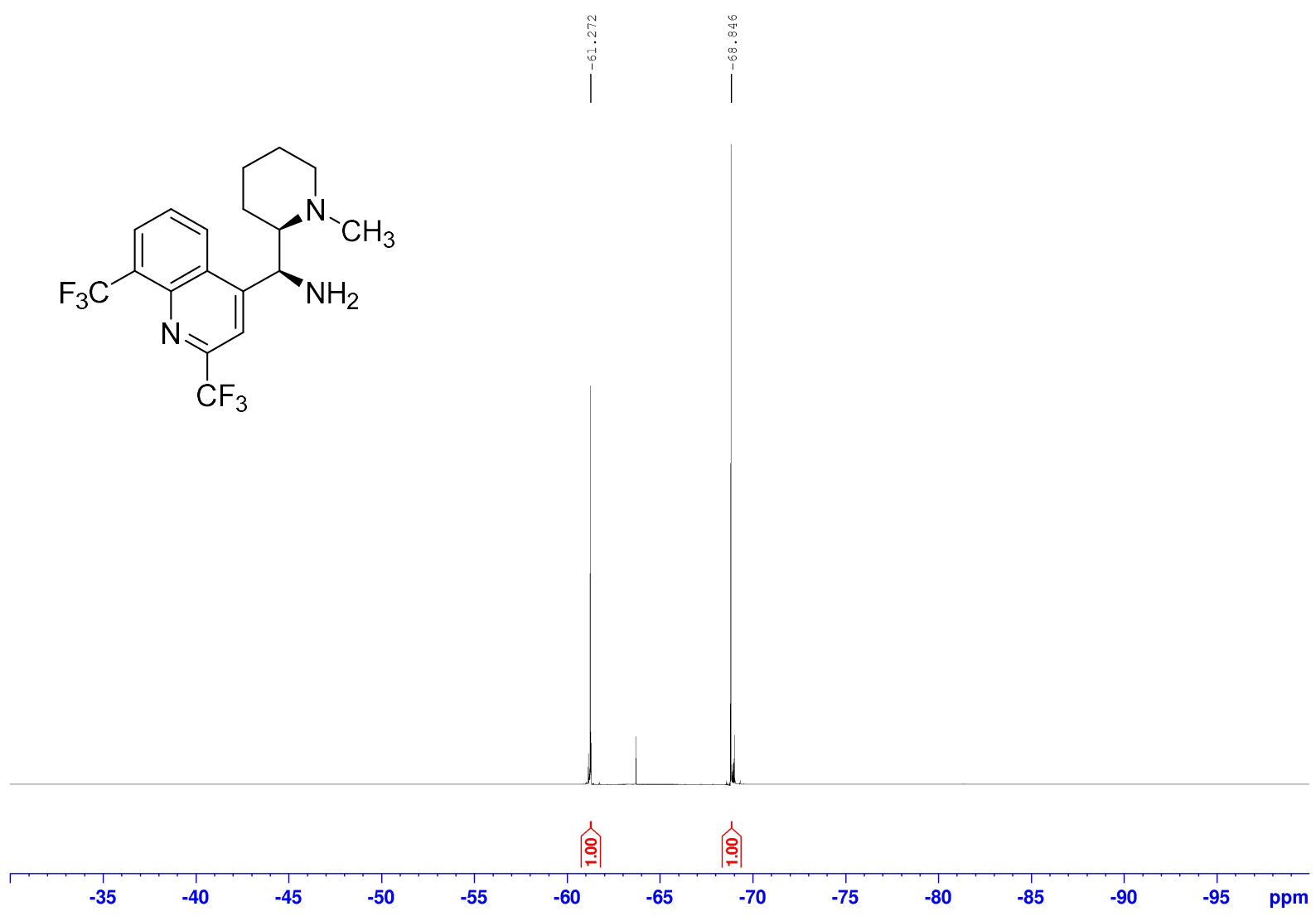

Figure S21. ${ }^{19} \mathrm{~F}$ NMR $(376 \mathrm{MHz})$ spectrum for $\mathbf{7 b}$ in $\mathrm{CDCl}_{3}+\mathrm{PhCF}_{3}\left(\delta_{\mathrm{F}}=-63.72 \mathrm{ppm}\right)$ 


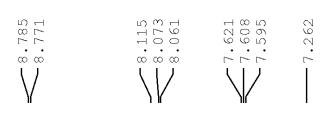

W wi

I Ywe<smiles>CC(F)(F)N1CCCCC1C(O)c1cc(C(F)(F)F)nc2c(C(F)(F)F)cccc12</smiles>

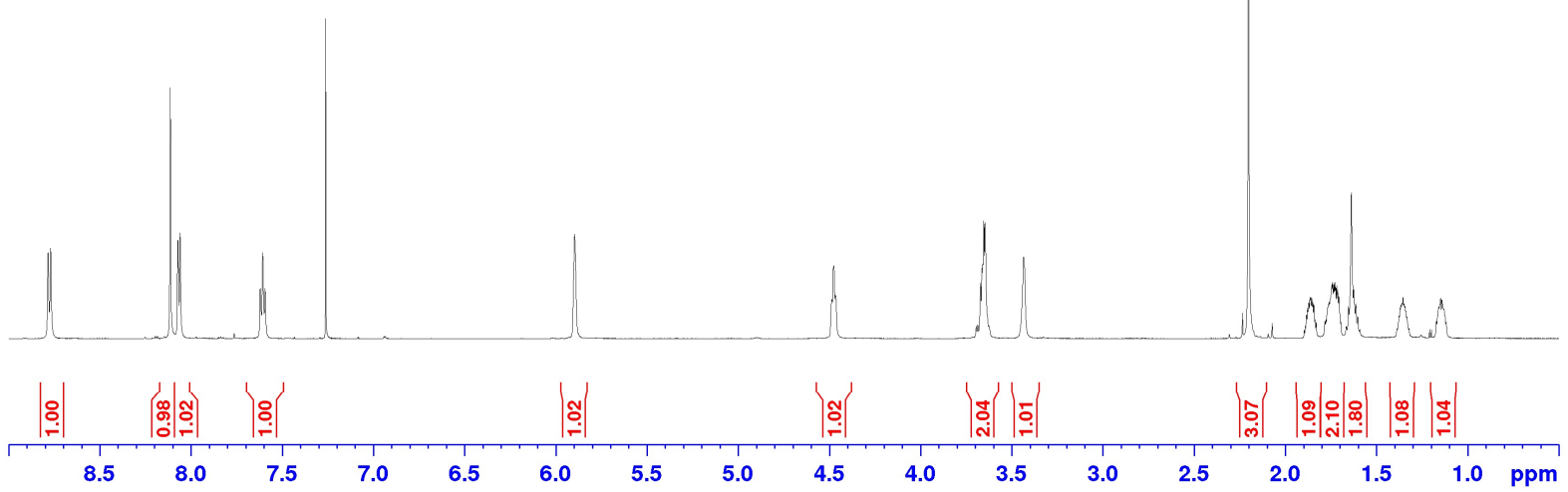

Figure S22. ${ }^{1} \mathrm{H}(600 \mathrm{MHz})$ spectrum for erythro-13-acetyl-mefloquine in $\mathrm{CDCl}_{3}+\mathrm{TMS}$ 


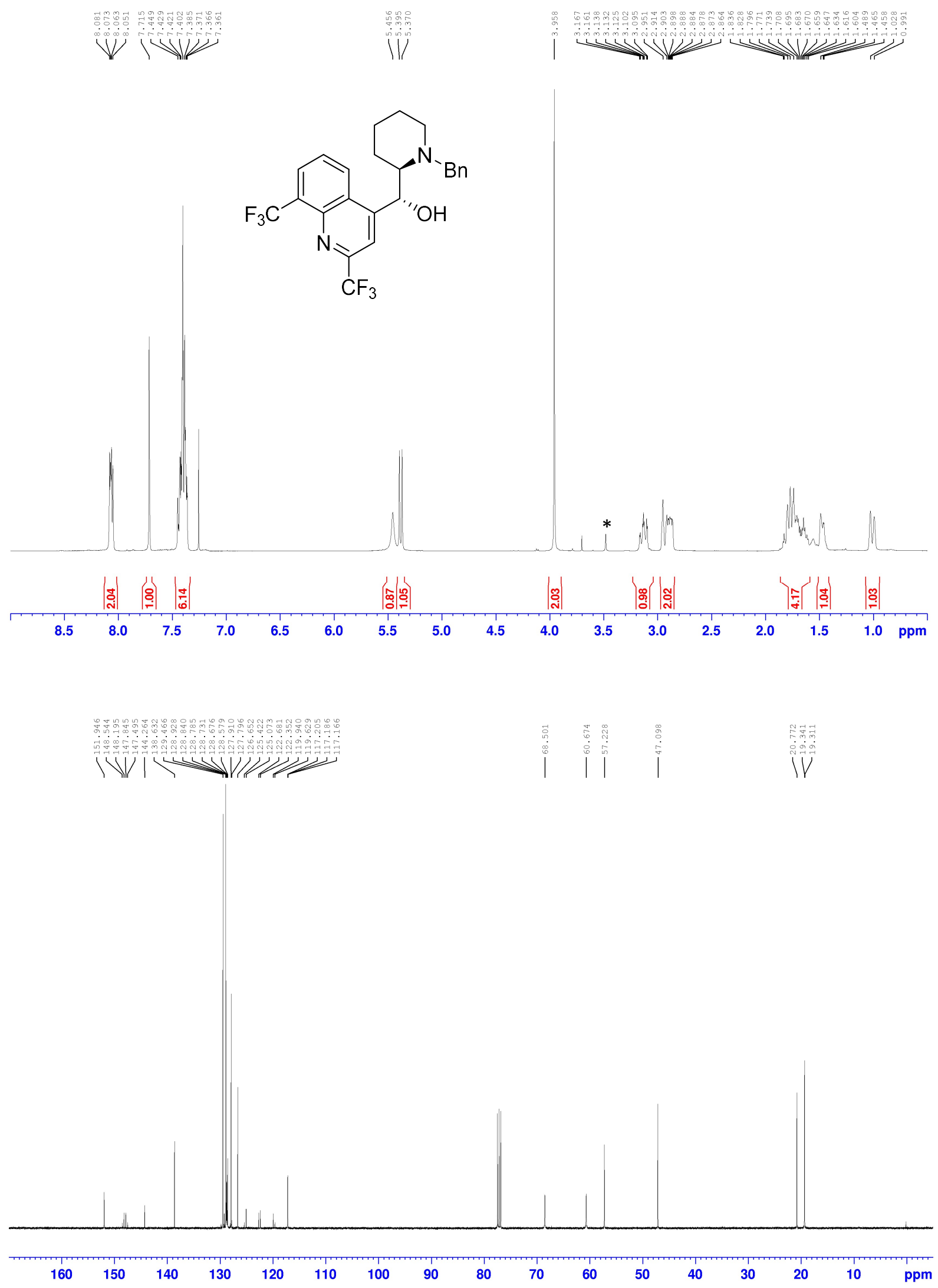

Figure S23. ${ }^{1} \mathrm{H}(400 \mathrm{MHz})$ and ${ }^{13} \mathrm{C}\left\{{ }^{1} \mathrm{H}\right\}$ NMR $(100 \mathrm{MHz})$ spectra for 9 in $\mathrm{CDCl}_{3}+\mathrm{TMS}$. (* trace of $\mathrm{MeOH}$ identified) 


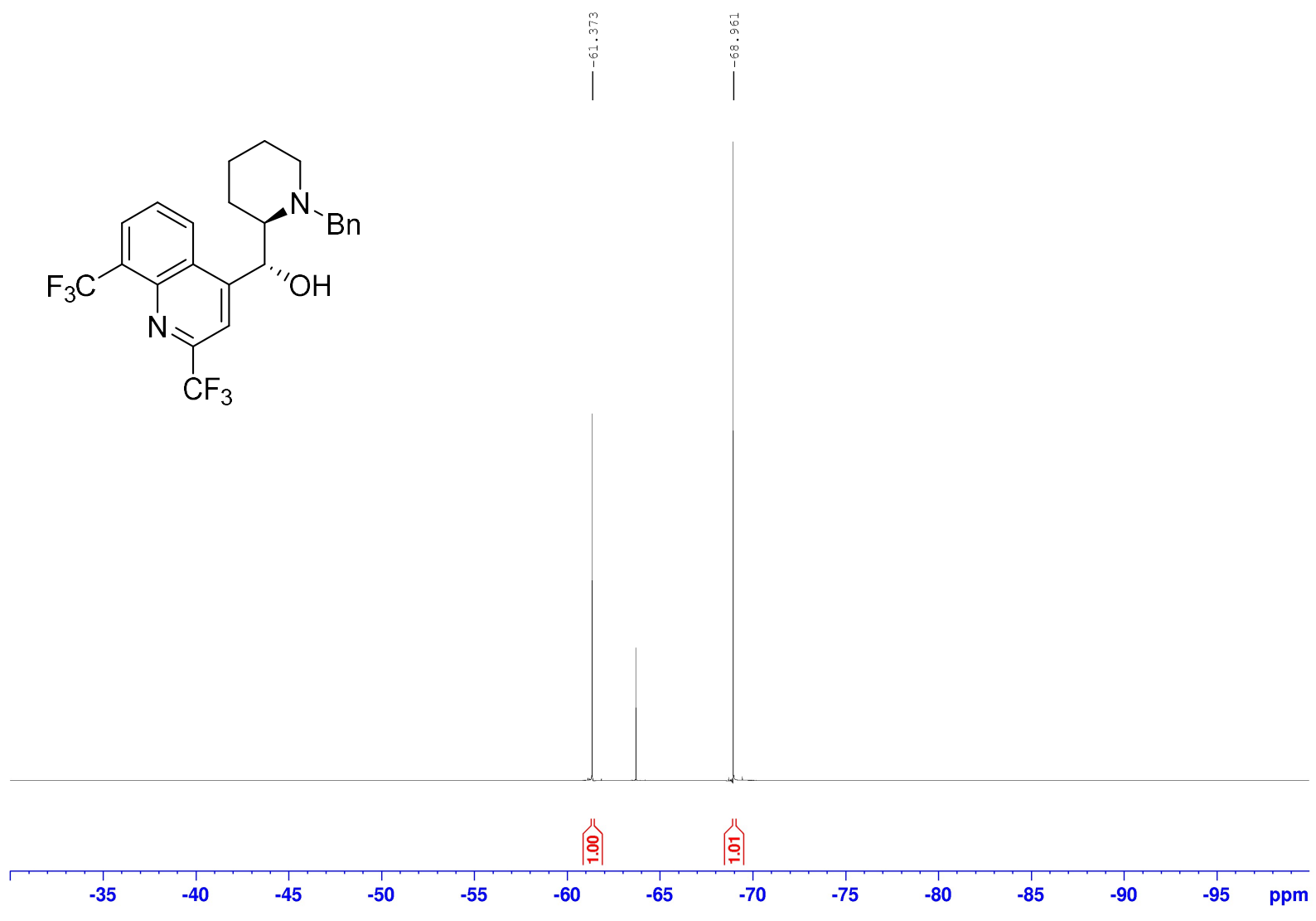

Figure S24. ${ }^{19} \mathrm{~F}$ NMR (376 MHz) spectrum for 9 in $\mathrm{CDCl}_{3}+\mathrm{PhCF}_{3}\left(\delta_{\mathrm{F}}=-63.72 \mathrm{ppm}\right)$ 

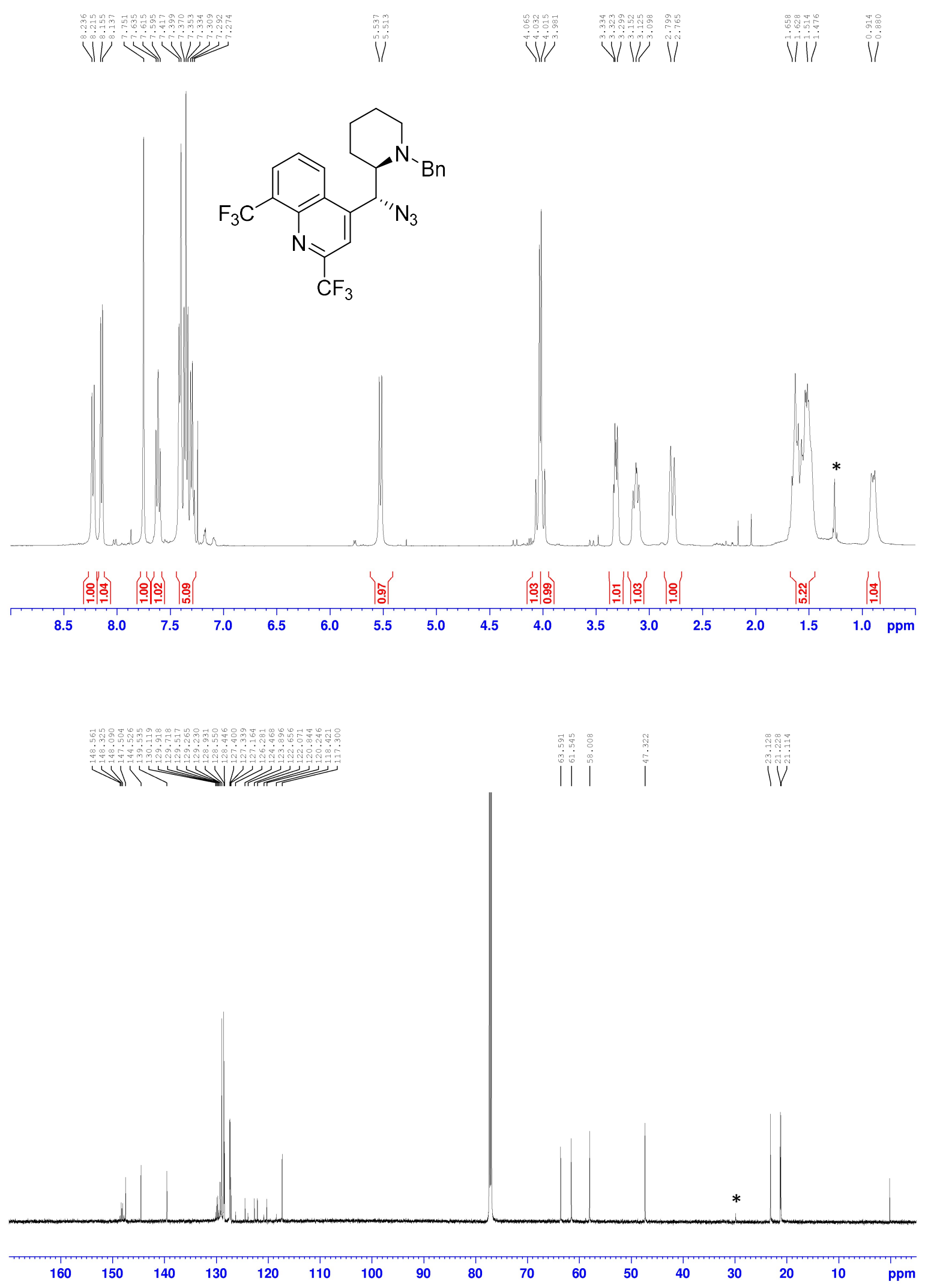

Figure S25. ${ }^{1} \mathrm{H}(400 \mathrm{MHz})$ and ${ }^{13} \mathrm{C}\left\{{ }^{1} \mathrm{H}\right\} \mathrm{NMR}(151 \mathrm{MHz})$ spectra for 10 in $\mathrm{CDCl}_{3}+\mathrm{TMS}\left({ }^{*}\right.$ trace of high boiling hydrocarbons identified) 


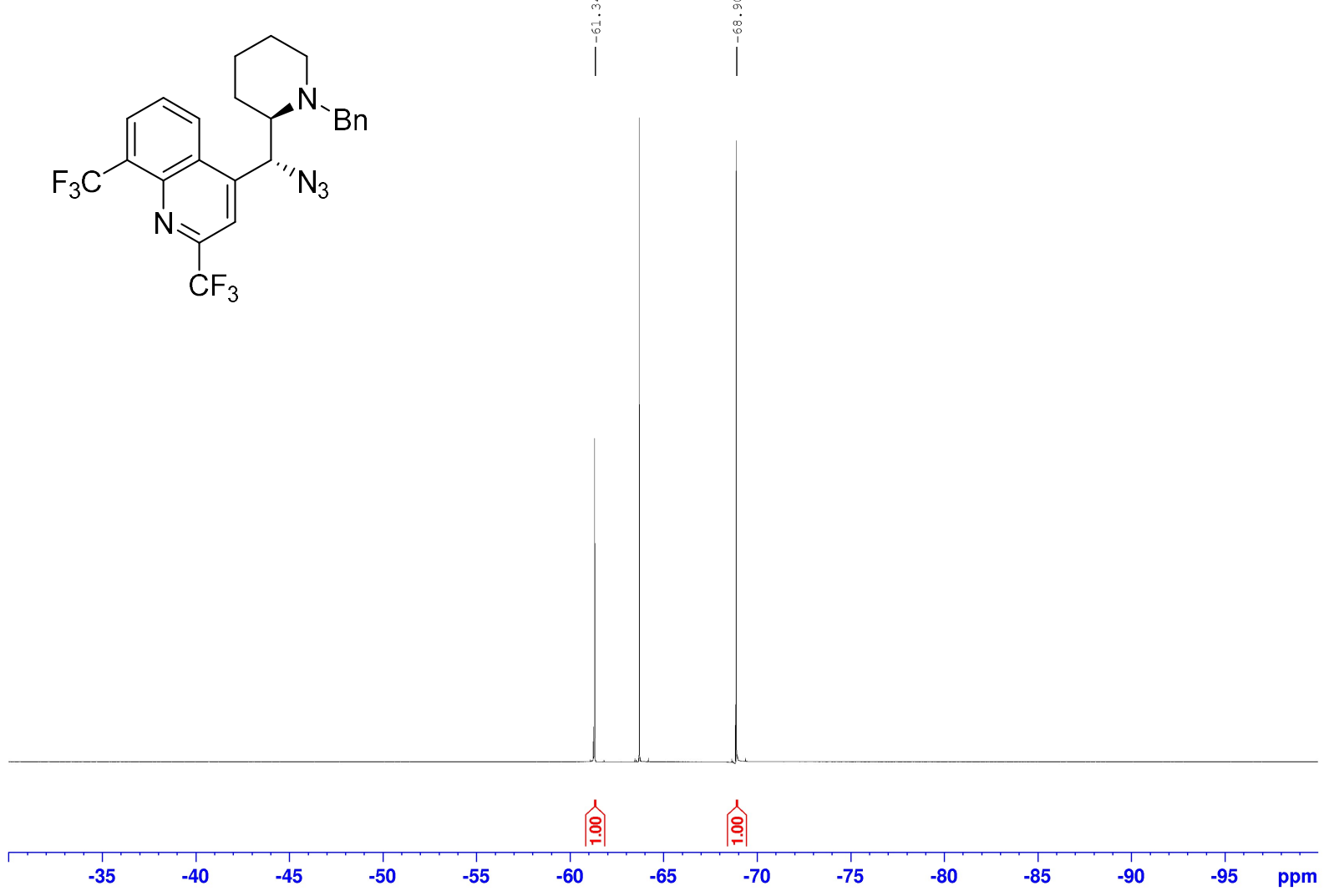

Figure S26. ${ }^{19} \mathrm{~F}$ NMR $(376 \mathrm{MHz})$ spectrum for 10 in $\mathrm{CDCl}_{3}+\mathrm{PhCF}_{3}\left(\delta_{\mathrm{F}}=-63.72 \mathrm{ppm}\right)$ 

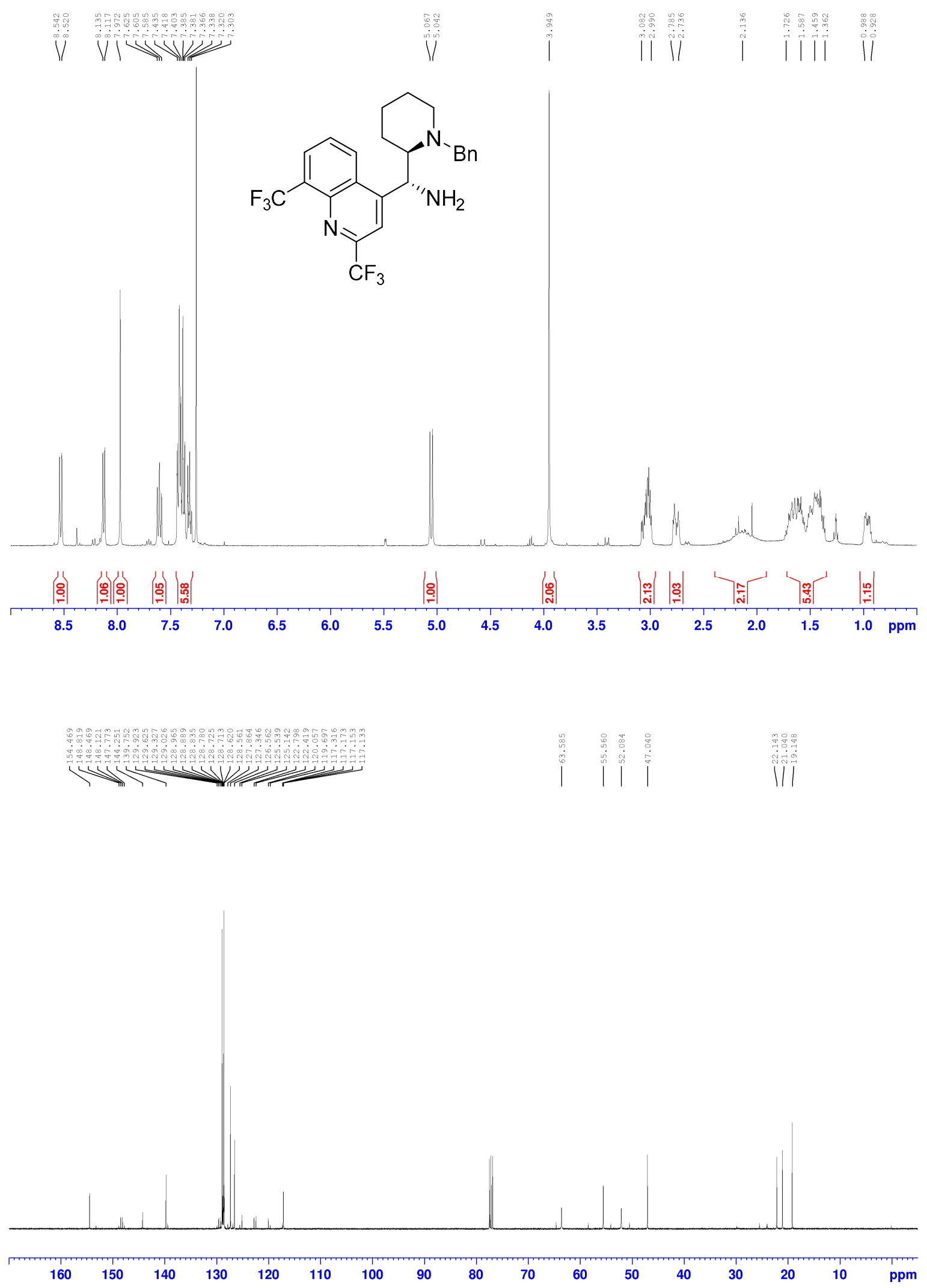

Figure S27. ${ }^{1} \mathrm{H}(400 \mathrm{MHz})$ and ${ }^{13} \mathrm{C}\left\{{ }^{1} \mathrm{H}\right\}$ NMR $(100 \mathrm{MHz})$ spectra for 11 in $\mathrm{CDCl}_{3}+\mathrm{TMS}$ 


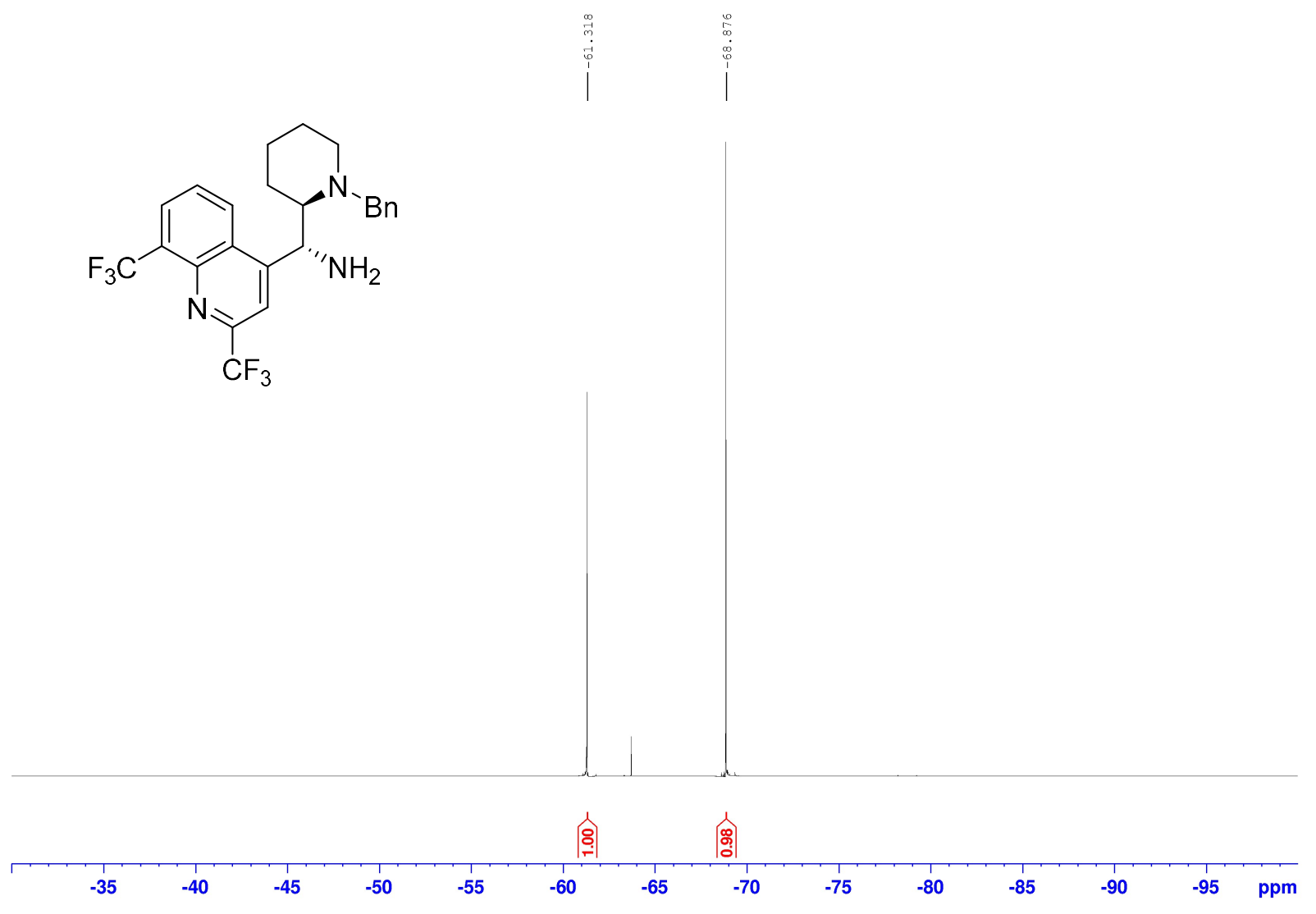

Figure S28. ${ }^{19} \mathrm{~F}$ NMR (376 MHz) spectrum for 11 in $\mathrm{CDCl}_{3}+\mathrm{PhCF}_{3}\left(\delta_{\mathrm{F}}=-63.72 \mathrm{ppm}\right)$ 


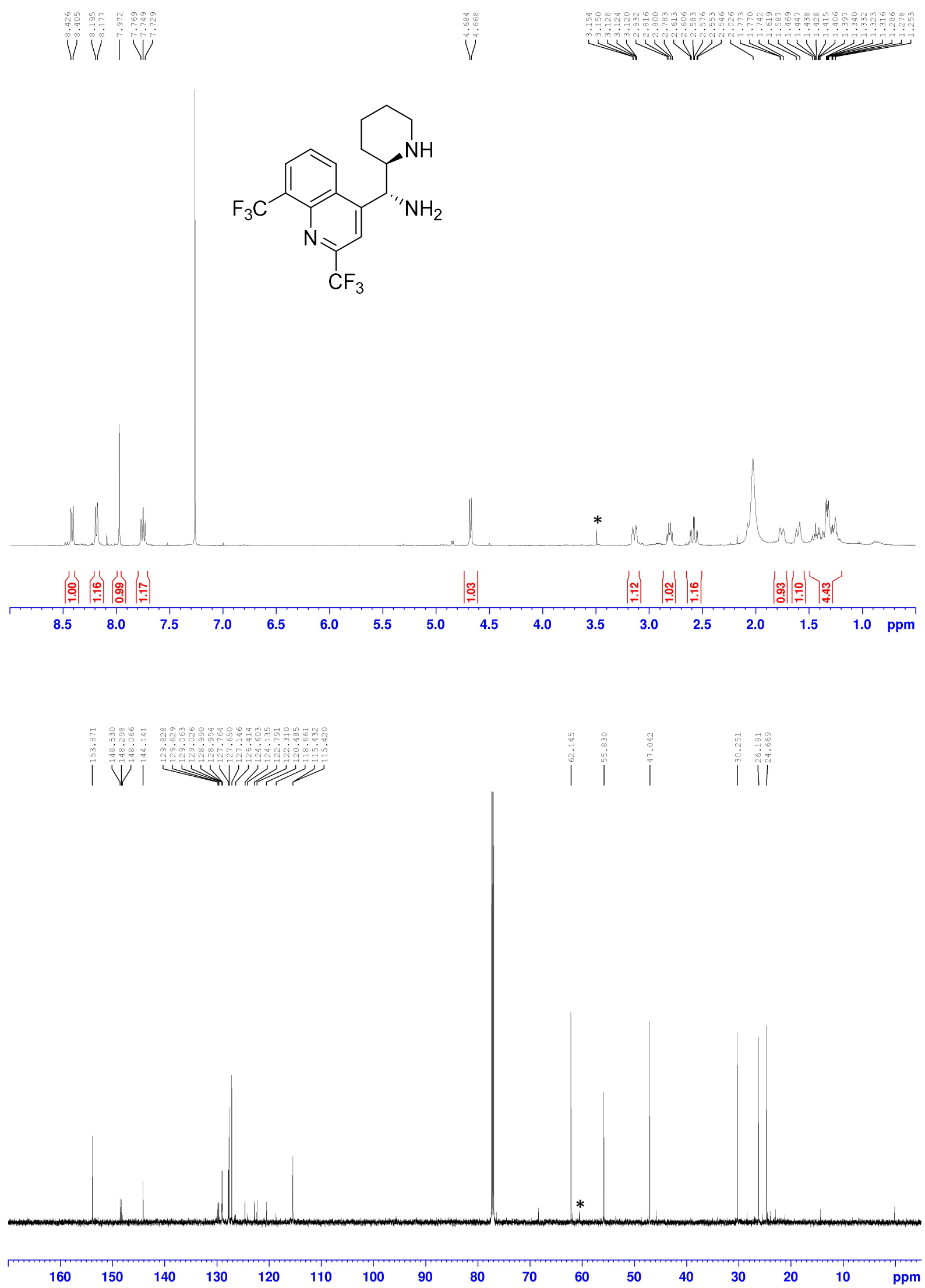

Figure S29. ${ }^{1} \mathrm{H}(400 \mathrm{MHz})$ and ${ }^{13} \mathrm{C}\left\{{ }^{1} \mathrm{H}\right\} \mathrm{NMR}(151 \mathrm{MHz})$ spectra for 12 in $\mathrm{CDCl}_{3}+\mathrm{TMS}$. (* trace of $\mathrm{MeOH}$ identified) 


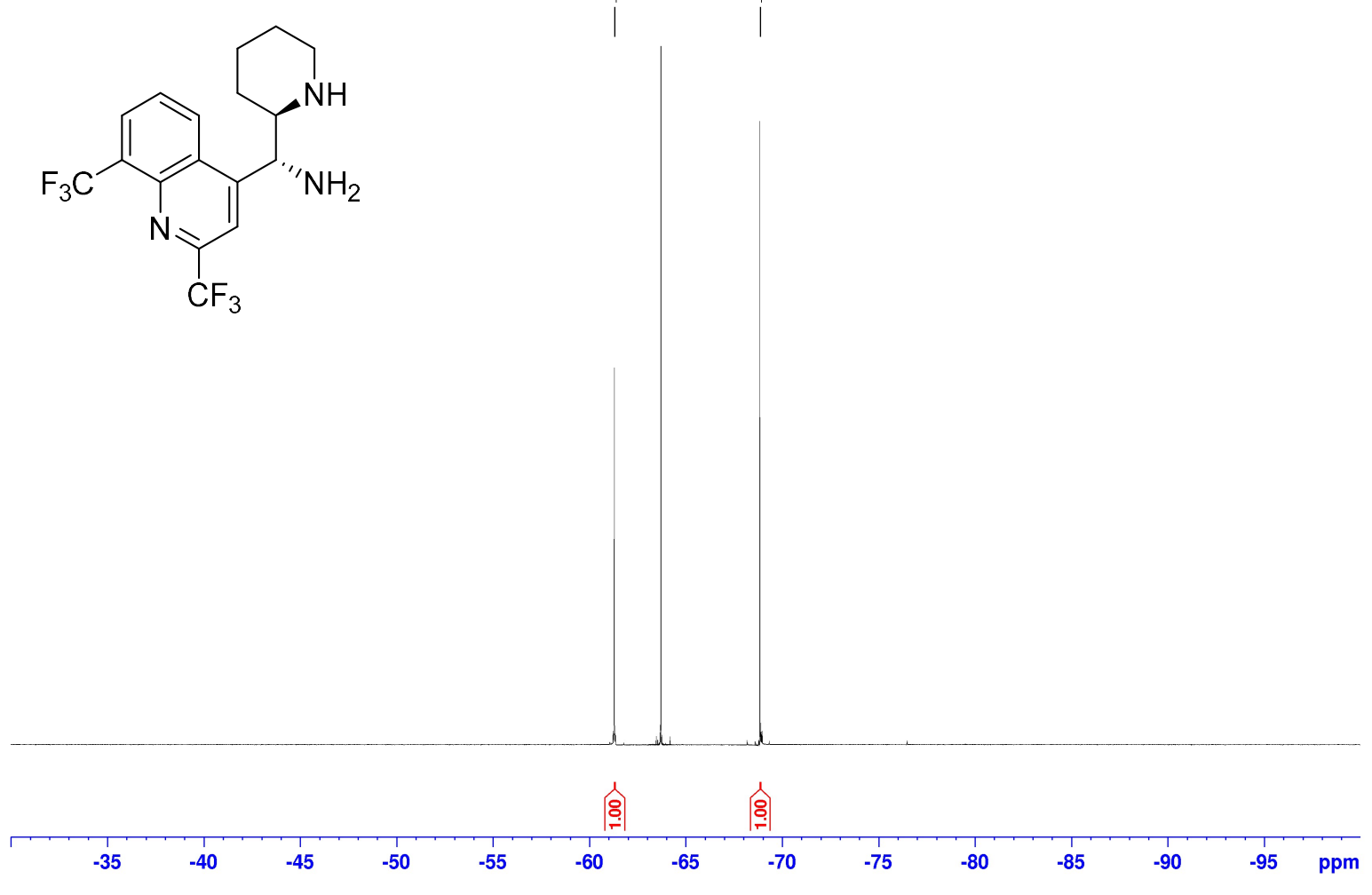

Figure S30. ${ }^{19} \mathrm{~F}$ NMR ( $\left.376 \mathrm{MHz}\right)$ spectrum for 12 in $\mathrm{CDCl}_{3}+\mathrm{PhCF}_{3}\left(\delta_{\mathrm{F}}=-63.72 \mathrm{ppm}\right)$ 

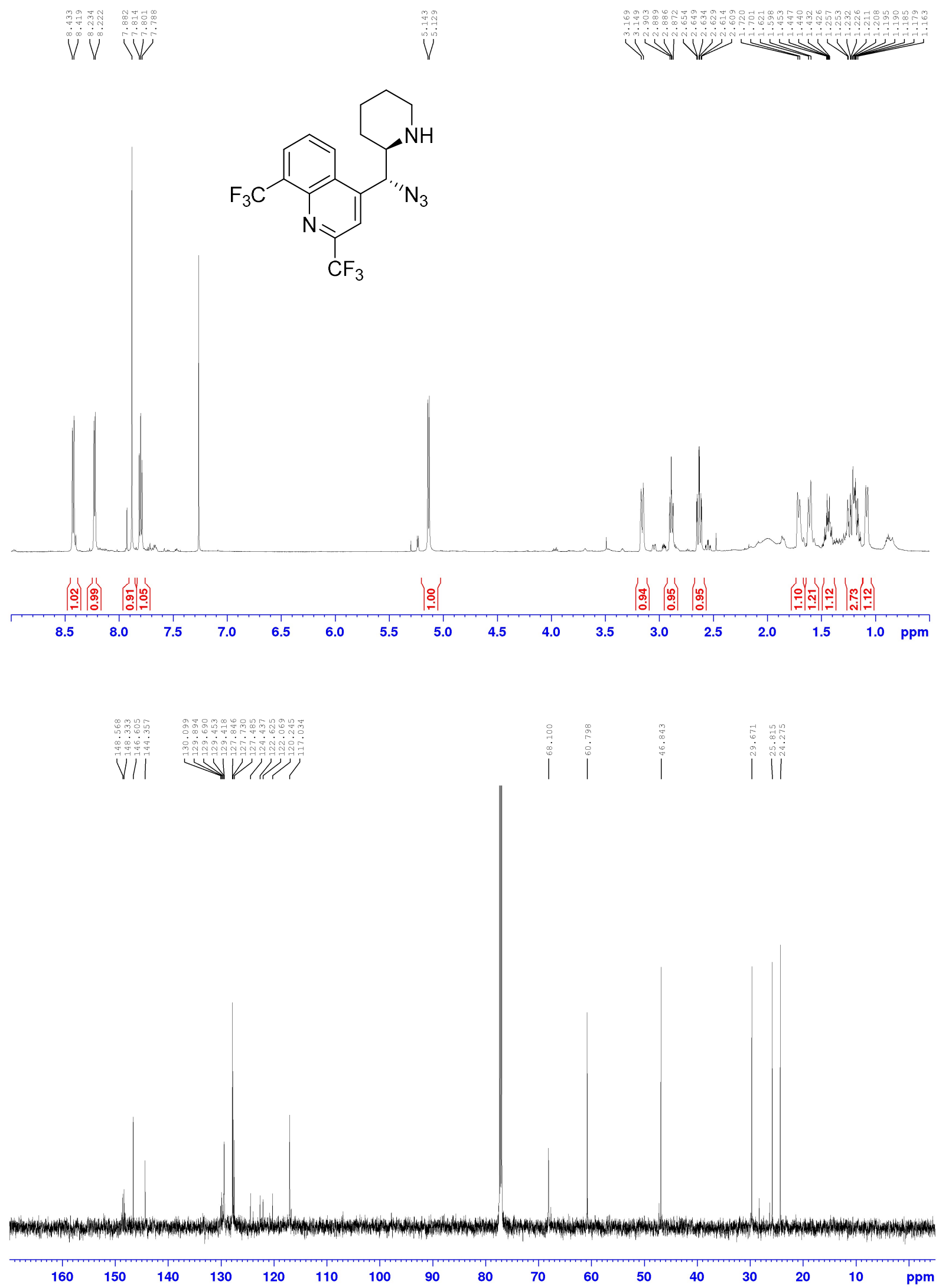

Figure S31. ${ }^{1} \mathrm{H}(600 \mathrm{MHz})$ and ${ }^{13} \mathrm{C}\left\{{ }^{1} \mathrm{H}\right\} \mathrm{NMR}(151 \mathrm{MHz})$ spectra for 13, diasteromeric ratio $95: 5$, in $\mathrm{CDCl}_{3}+\mathrm{TMS}$. For spectra of diasteromer, see Figures S4-S5. 

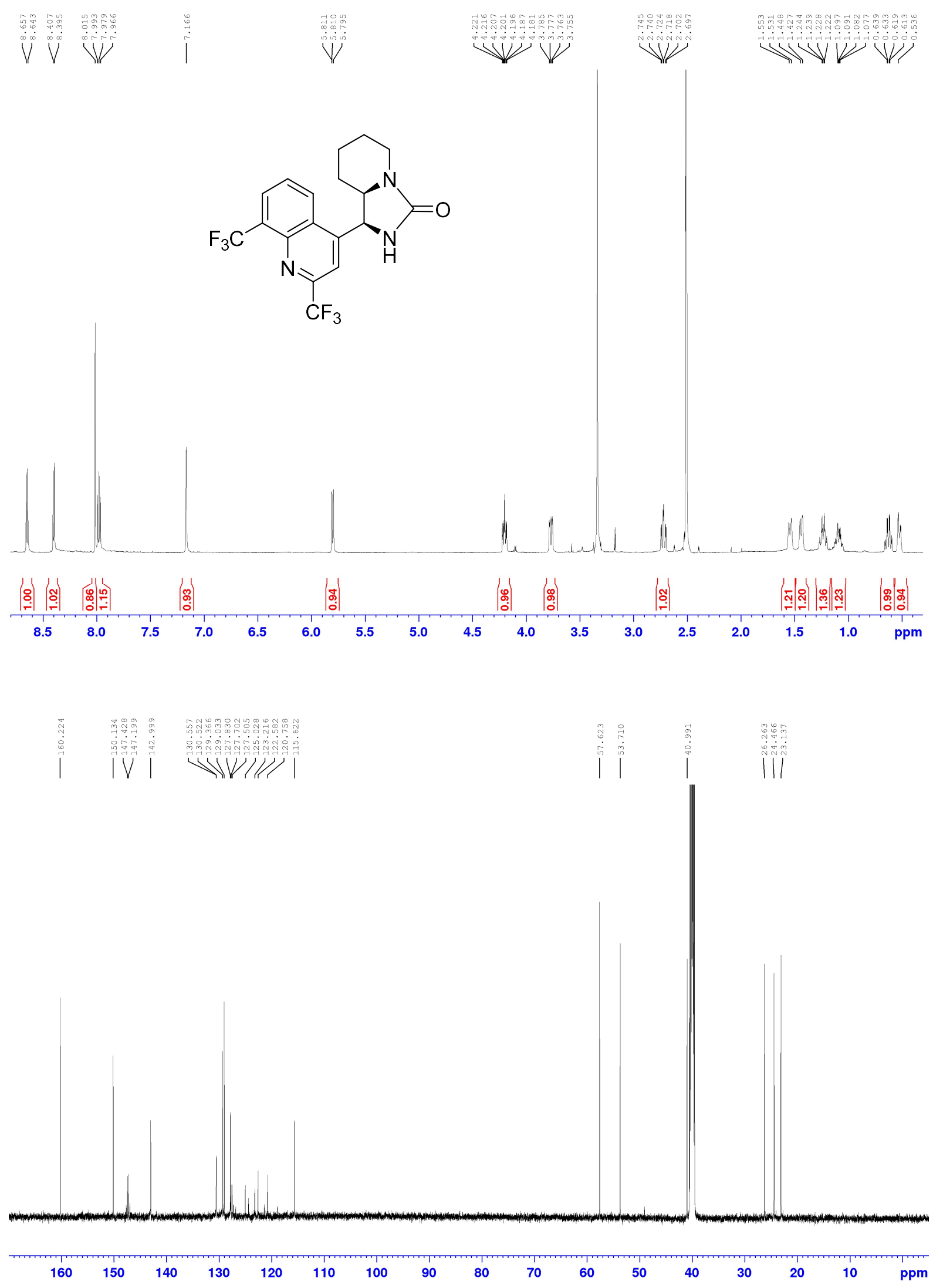

Figure S32. ${ }^{1} \mathrm{H}(600 \mathrm{MHz})$ and ${ }^{13} \mathrm{C}\left\{{ }^{1} \mathrm{H}\right\} \mathrm{NMR}(151 \mathrm{MHz})$ spectra for 14 in DMSO- $d_{6}+\mathrm{TMS}$ 


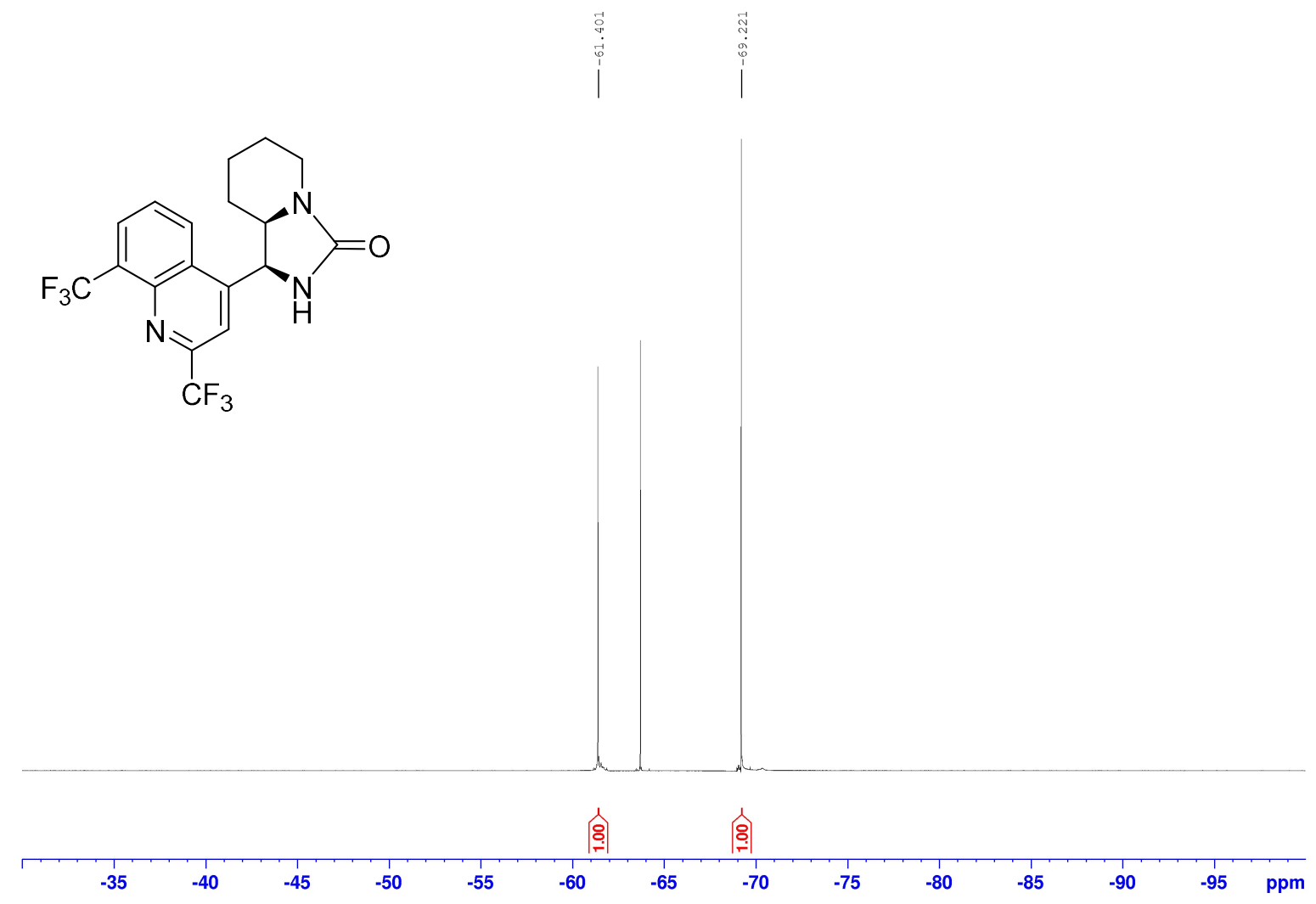

Figure S33. ${ }^{19} \mathrm{~F}$ NMR (376 MHz) spectrum for 14 in $\mathrm{CDCl}_{3}+\mathrm{PhCF}_{3}\left(\delta_{\mathrm{F}}=-63.72 \mathrm{ppm}\right)$ 

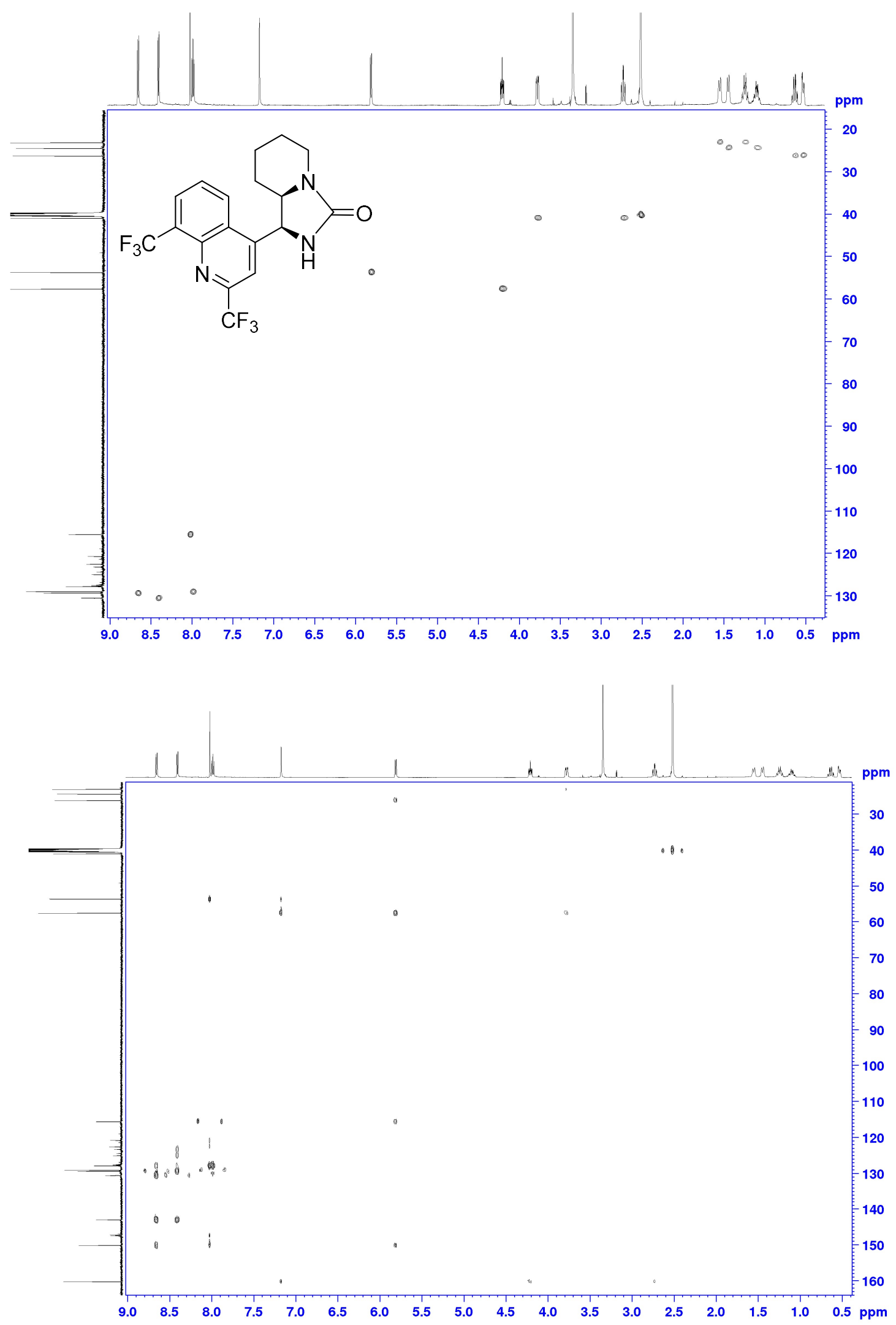

Figure S34. ${ }^{1} \mathrm{H},{ }^{13} \mathrm{C}$ HSQC (top) and HMBC (bottom) experiments for 14 in DMSO- $d_{6}$. For spectral assignment, see Figure S2. 

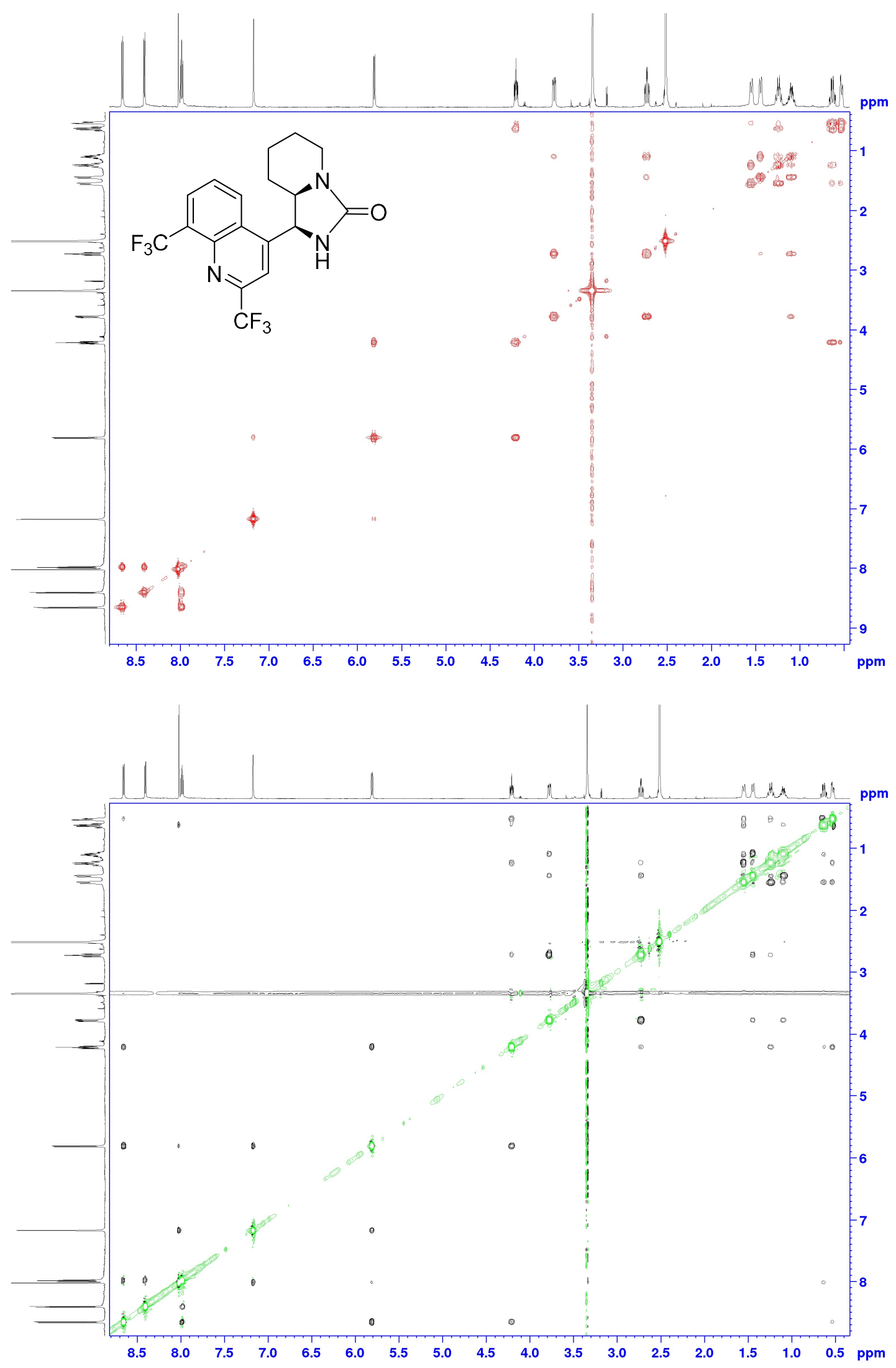

Figure S35. ${ }^{1} \mathrm{H},{ }^{1} \mathrm{H}$ COSY (top) and NOESY (bottom, phases of opposite signs are drawn in black and green) experiments for $\mathbf{1 4}$ in DMSO- $d_{6}$. For spectral assignment, see Figure S2. 


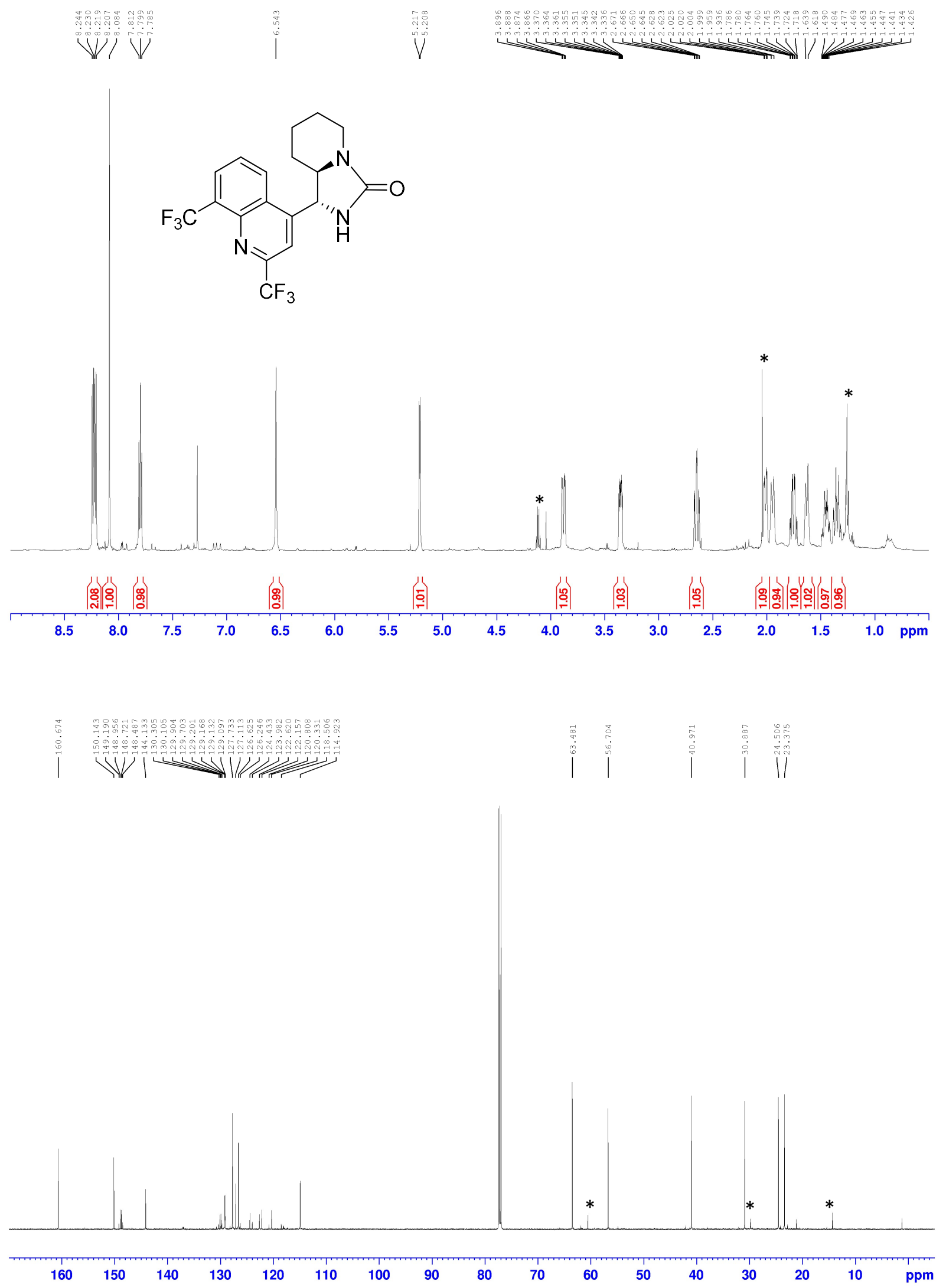

Figure S36. ${ }^{1} \mathrm{H}(600 \mathrm{MHz})$ and ${ }^{13} \mathrm{C}\left\{{ }^{1} \mathrm{H}\right\}$ NMR $(151 \mathrm{MHz})$ spectra for 15 in $\mathrm{CDCl}_{3}+\mathrm{TMS}$. (*trace of EtOAc was identified) 


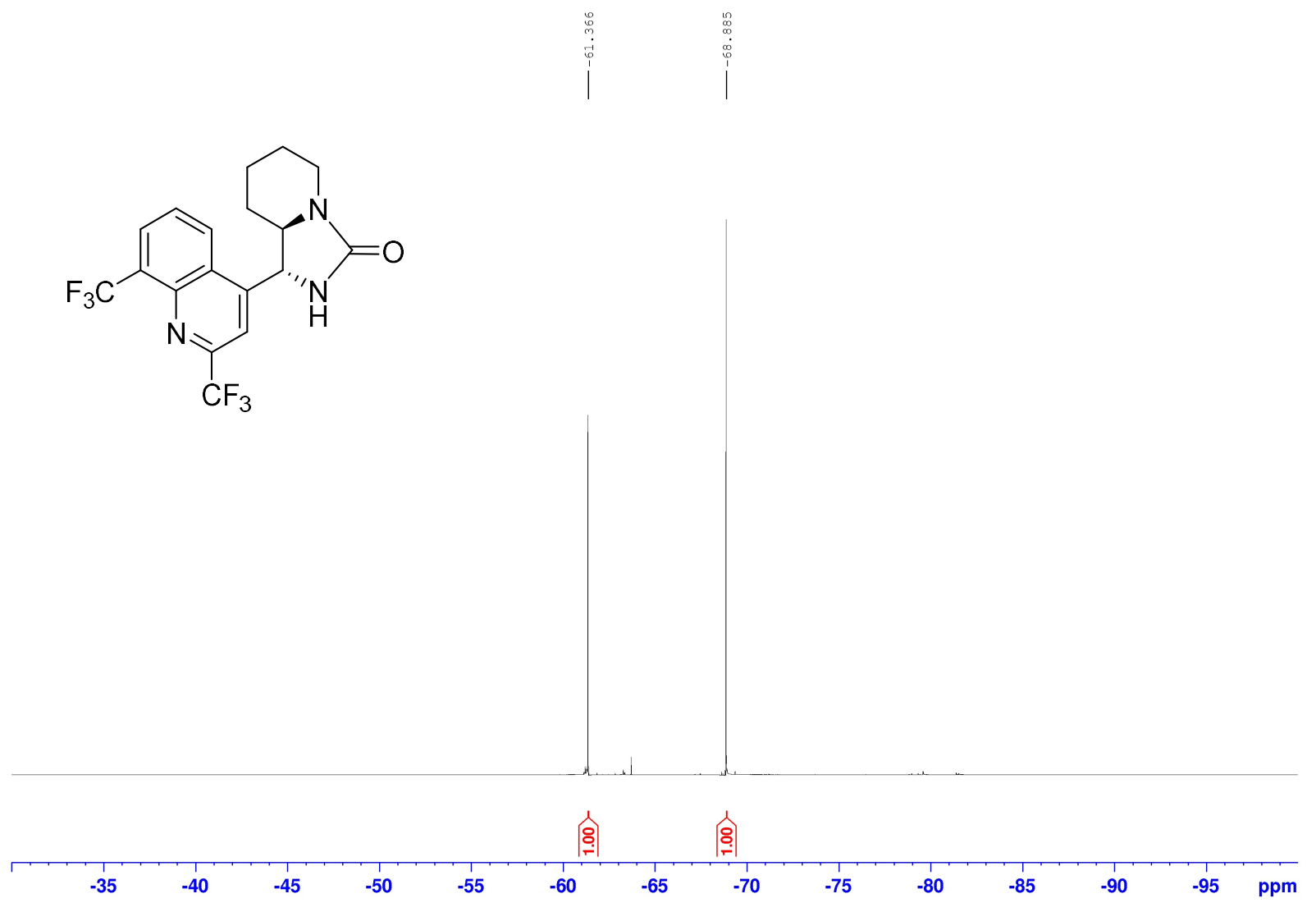

Figure S37. ${ }^{19} \mathrm{~F}$ NMR (376 MHz) spectrum for 15 in $\mathrm{CDCl}_{3}+\mathrm{PhCF}_{3}\left(\delta_{\mathrm{F}}=-63.72 \mathrm{ppm}\right)$ 

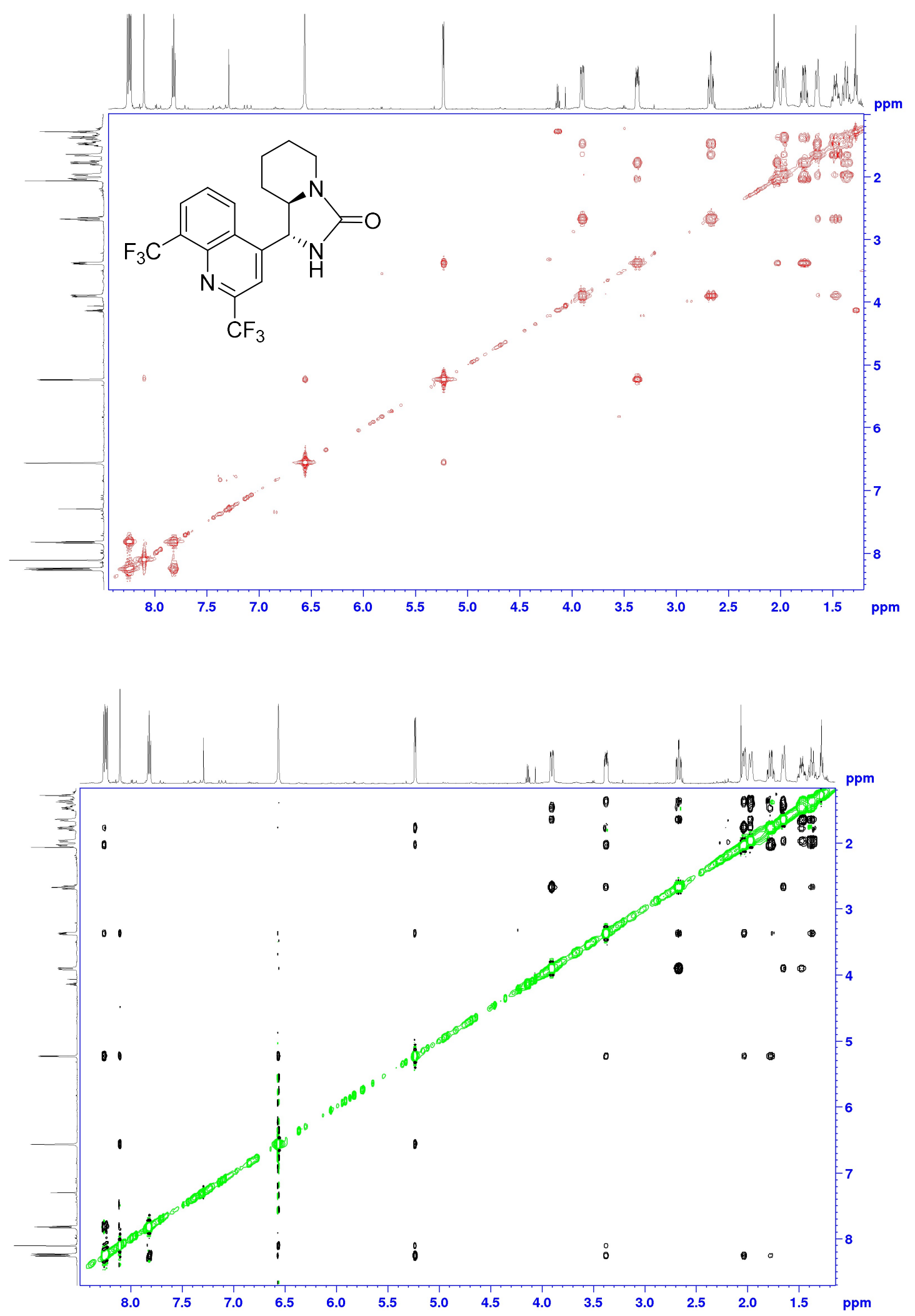

Figure S38. ${ }^{1} \mathrm{H},{ }^{1} \mathrm{H}$ COSY (top) and NOESY (bottom, phases of opposite signs are drawn in black and green) experiments for $\mathbf{1 5}$ in $\mathrm{CDCl}_{3}$. For spectral assignment, see Figure S2. 

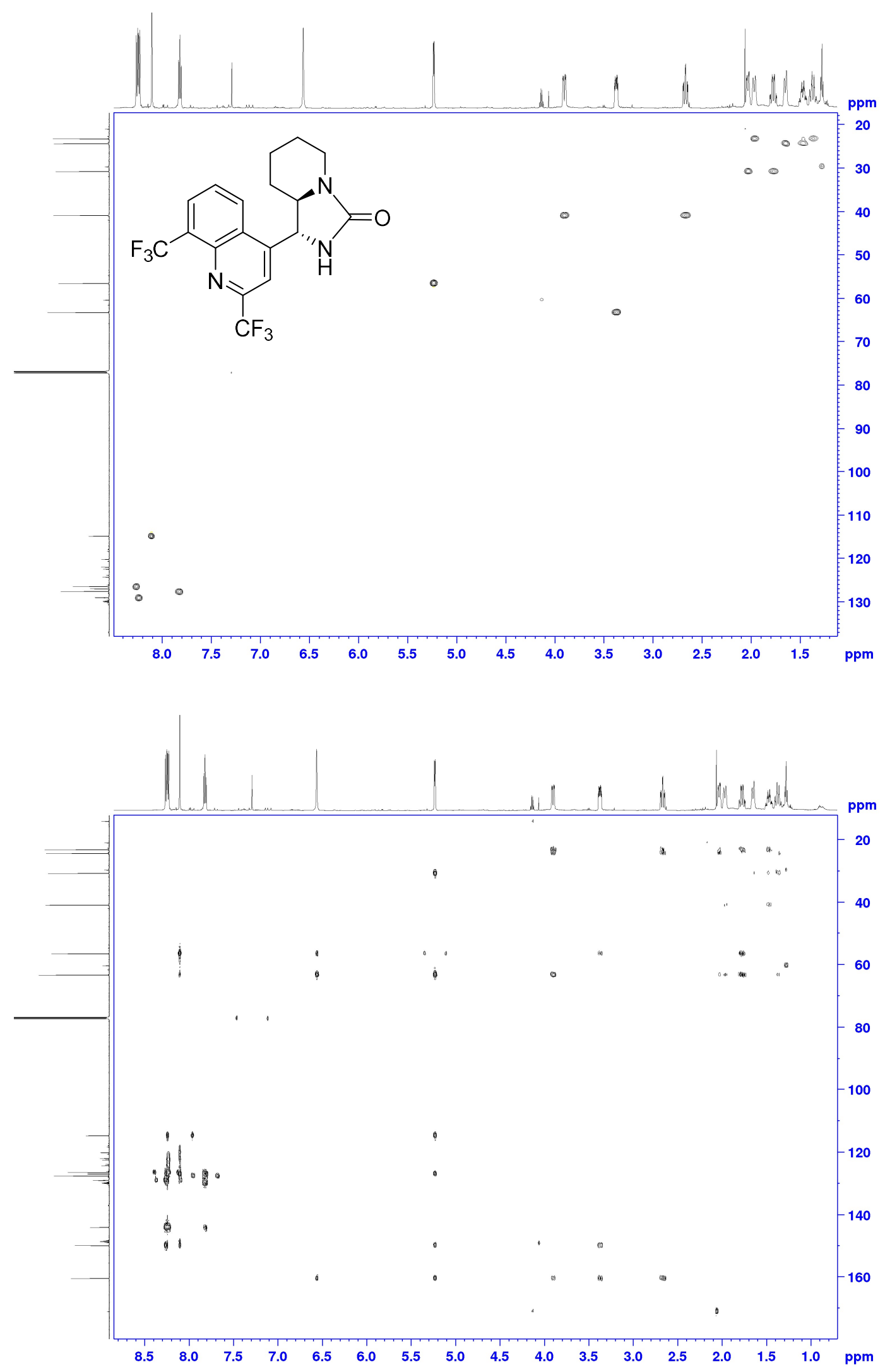

Figure S39. ${ }^{1} \mathrm{H},{ }^{13} \mathrm{C} \mathrm{HSQC}$ (top) and $\mathrm{HMBC}$ (bottom) experiments for 15 in $\mathrm{CDCl}_{3}$. For spectral assignment, see Figure S2. 


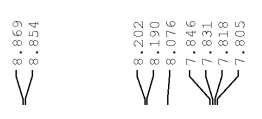<smiles>CN(C)[C@H](N)c1cc(C(F)(F)F)nc2c(C(F)(F)F)cccc12</smiles>
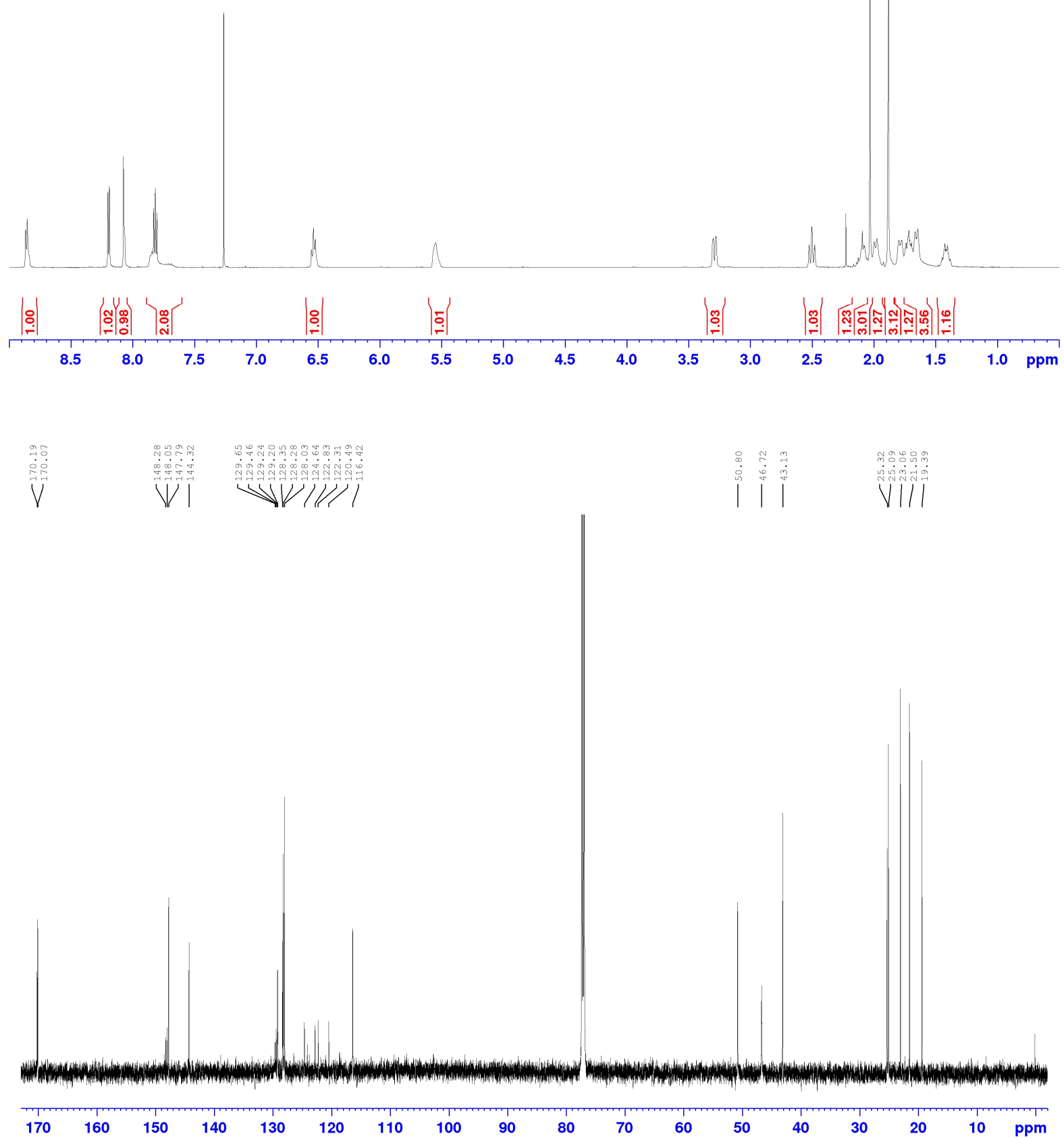

Figure S40. ${ }^{1} \mathrm{H}(600 \mathrm{MHz})$ and ${ }^{13} \mathrm{C}\left\{{ }^{1} \mathrm{H}\right\}$ NMR $(151 \mathrm{MHz})$ spectra for 16 in $\mathrm{CDCl}_{3}+\mathrm{TMS}$ 
<smiles>CC(C)N1CCCCC1[C@H](NC(C)(F)F)c1cc(C(F)(F)F)nc2c(C(F)(F)F)cccc12</smiles>

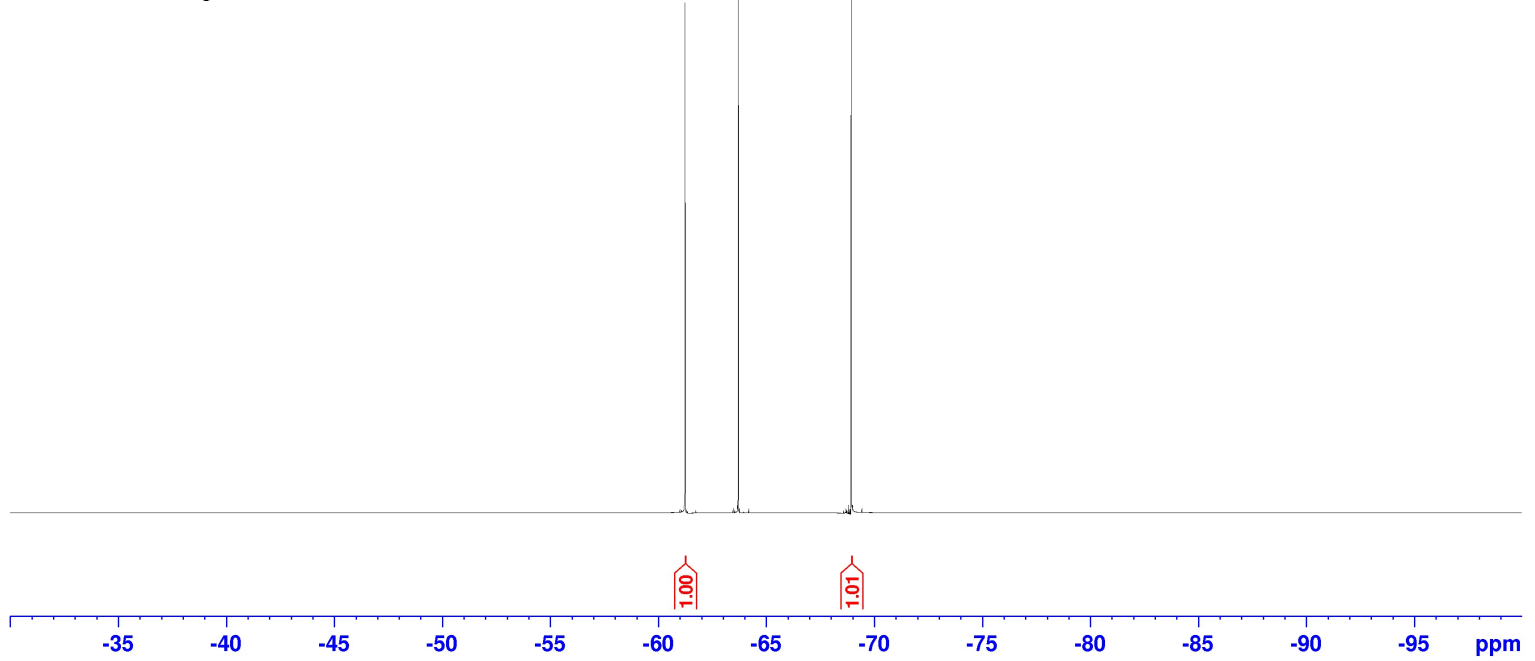

Figure S41. ${ }^{19} \mathrm{~F}$ NMR (376 MHz) spectrum for 16 in $\mathrm{CDCl}_{3}+\mathrm{PhCF}_{3}\left(\delta_{\mathrm{F}}=-63.72 \mathrm{ppm}\right)$ 


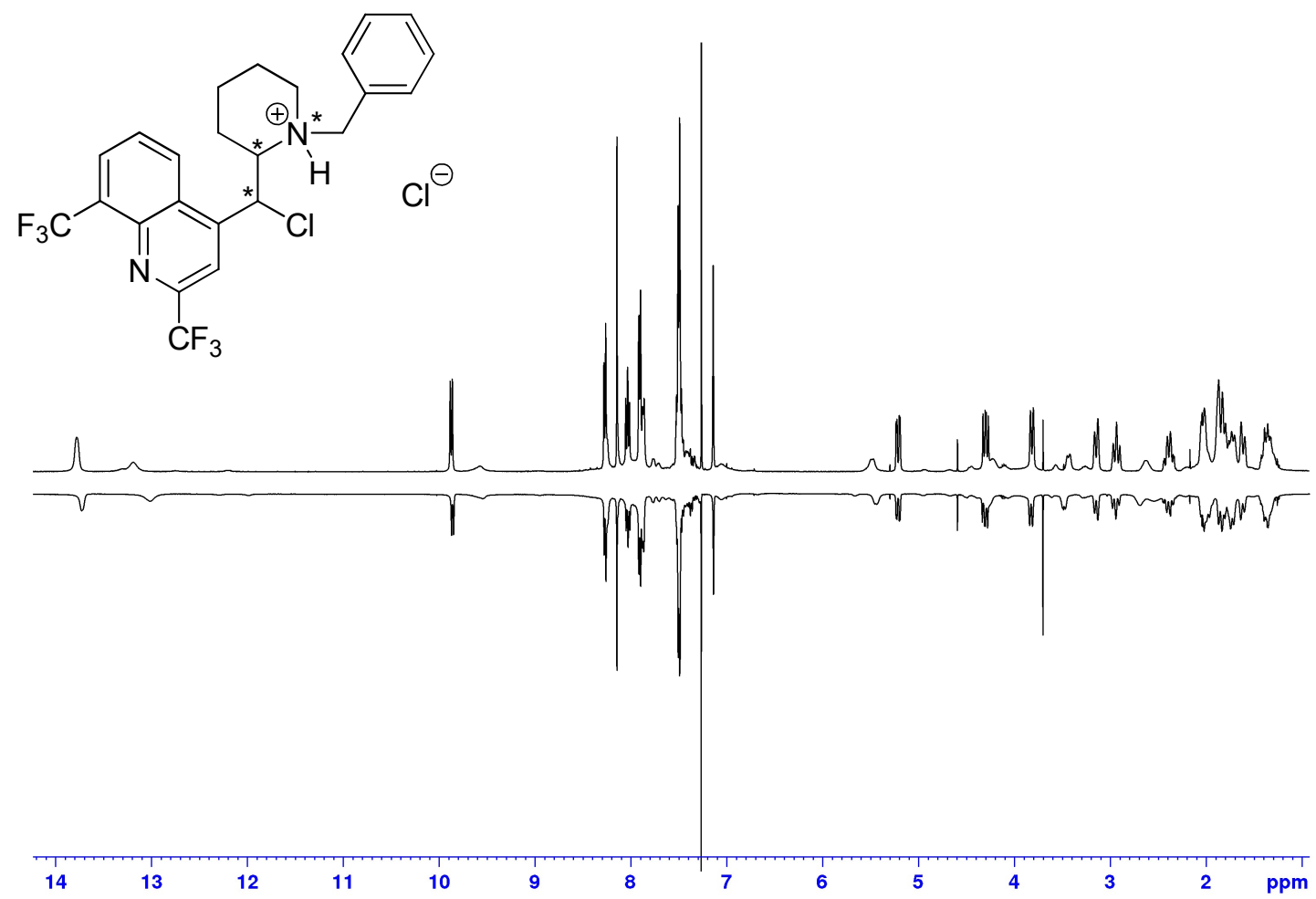

Figure S42. ${ }^{1} \mathrm{H}$ NMR ( $400 \mathrm{MHz}, \mathrm{CDCl}_{3}$ ) spectra for 11-chloroderivatives obtained after thionyl chloride treatment of 13-benzyl-mefloquine of erythro configuration (5a, positive phase) and threo configuration (9, negative phase).

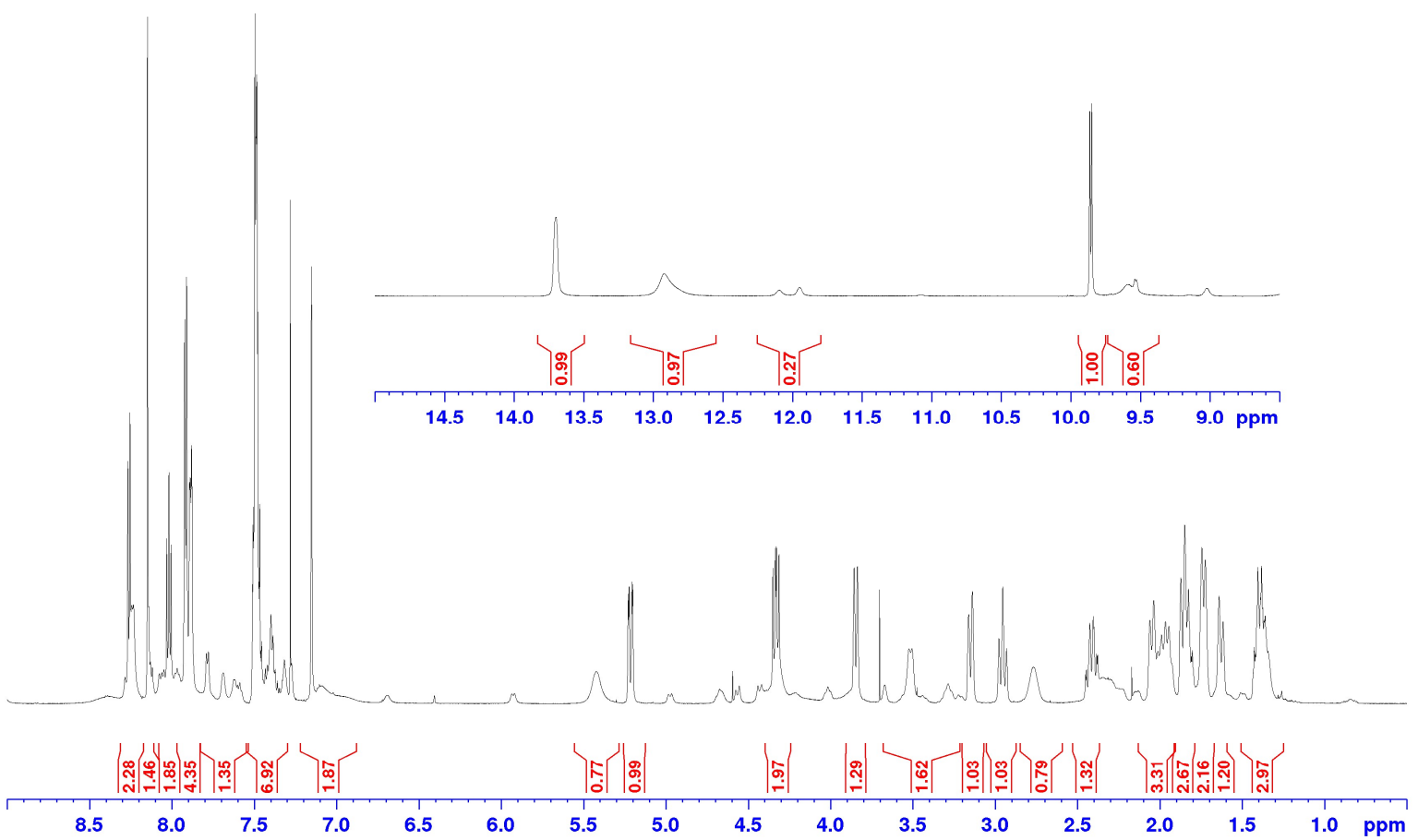

Figure S43. ${ }^{1} \mathrm{H}$ NMR $\left(600 \mathrm{MHz}, \mathrm{CDCl}_{3}\right)$ spectra for 11-chloroderivatives obtained after thionyl chloride treatment of 13-benzyl-mefloquine. See preceding Figure S42 for comparison of samples obtained from different starting materials. 

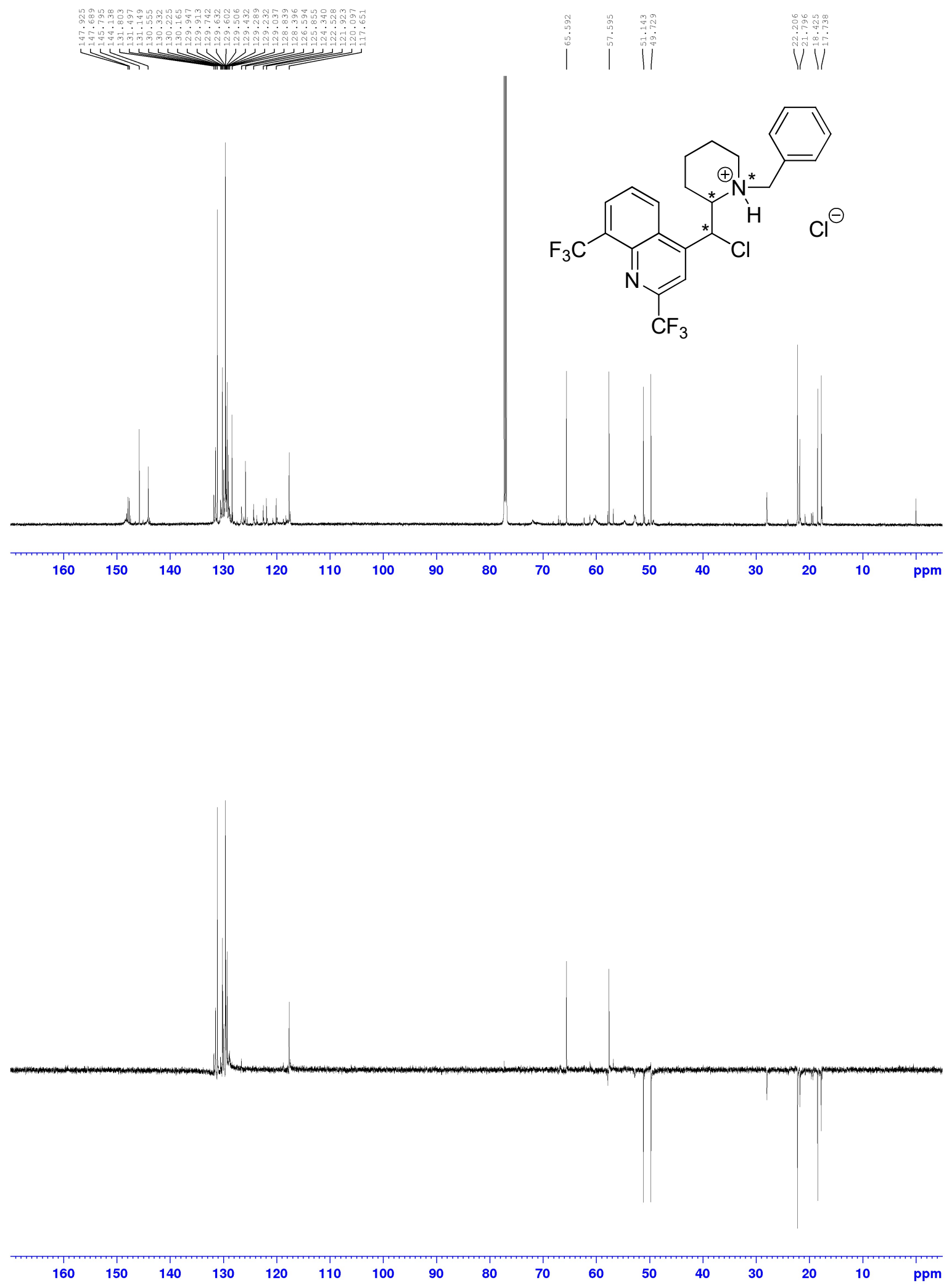

Figure S44. ${ }^{13} \mathrm{C}\left\{{ }^{1} \mathrm{H}\right\}$ and ${ }^{13} \mathrm{C}$ DEPT- $135^{\circ}$ NMR experiments (151 MHz, $\left.\mathrm{CDCl}_{3}\right)$ for likely 11 chloroderivatives obtained after thionyl chloride treatment of 13-benzyl-mefloquine 


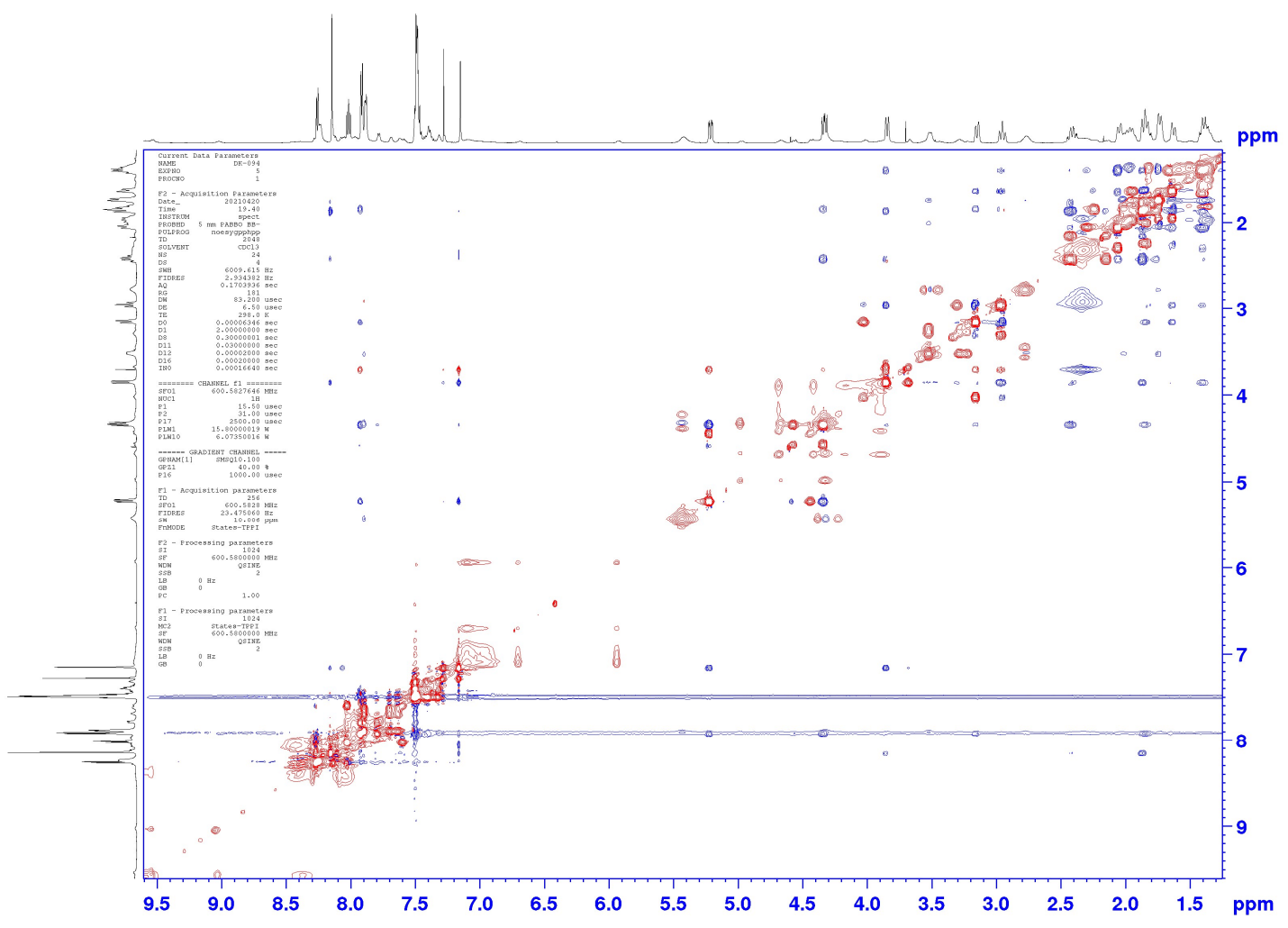

Figure S45. ${ }^{1} \mathrm{H}$ NOESY/EXSY experiment (600 MHz, $\mathrm{CDCl}_{3}$, TMS) for 11-chloroderivatives obtained after thionyl chloride treatment of 13-benzyl-mefloquine. Positive phase correlations are drawn in red (diagonal and EXSY) and negative phase correlations are drawn in blue (NOE). Considered equilibrating species include a series of rotamers/conformers and epimers at nitrogen atom shown below:

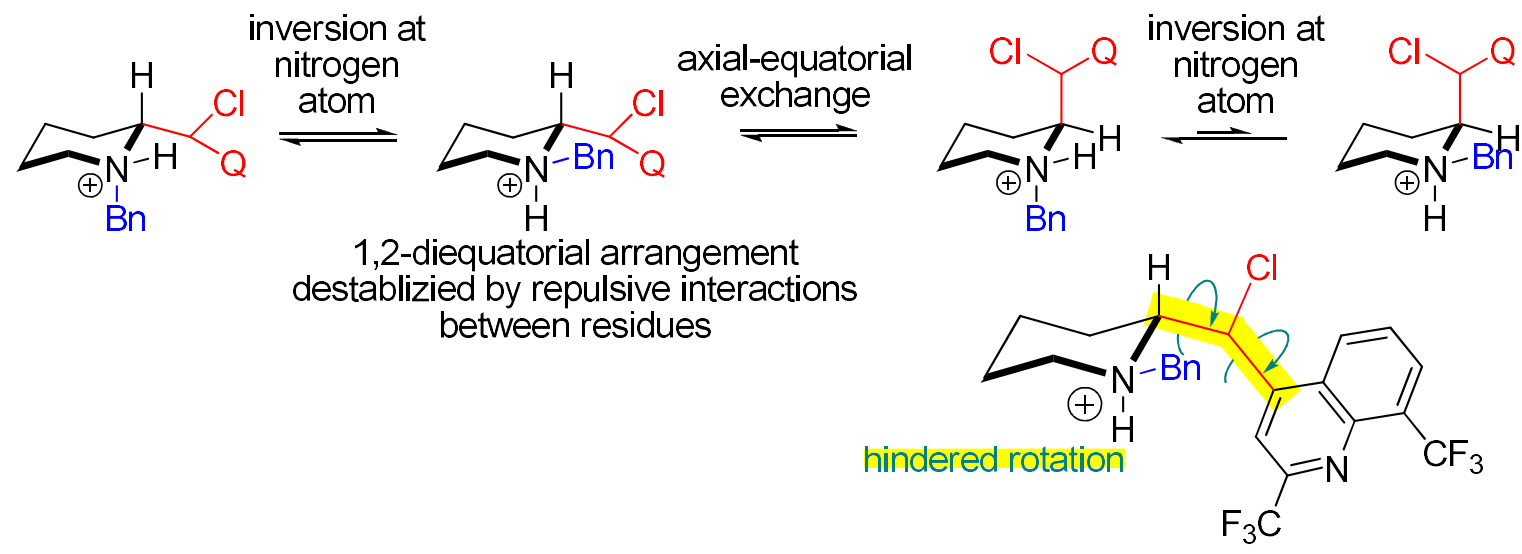




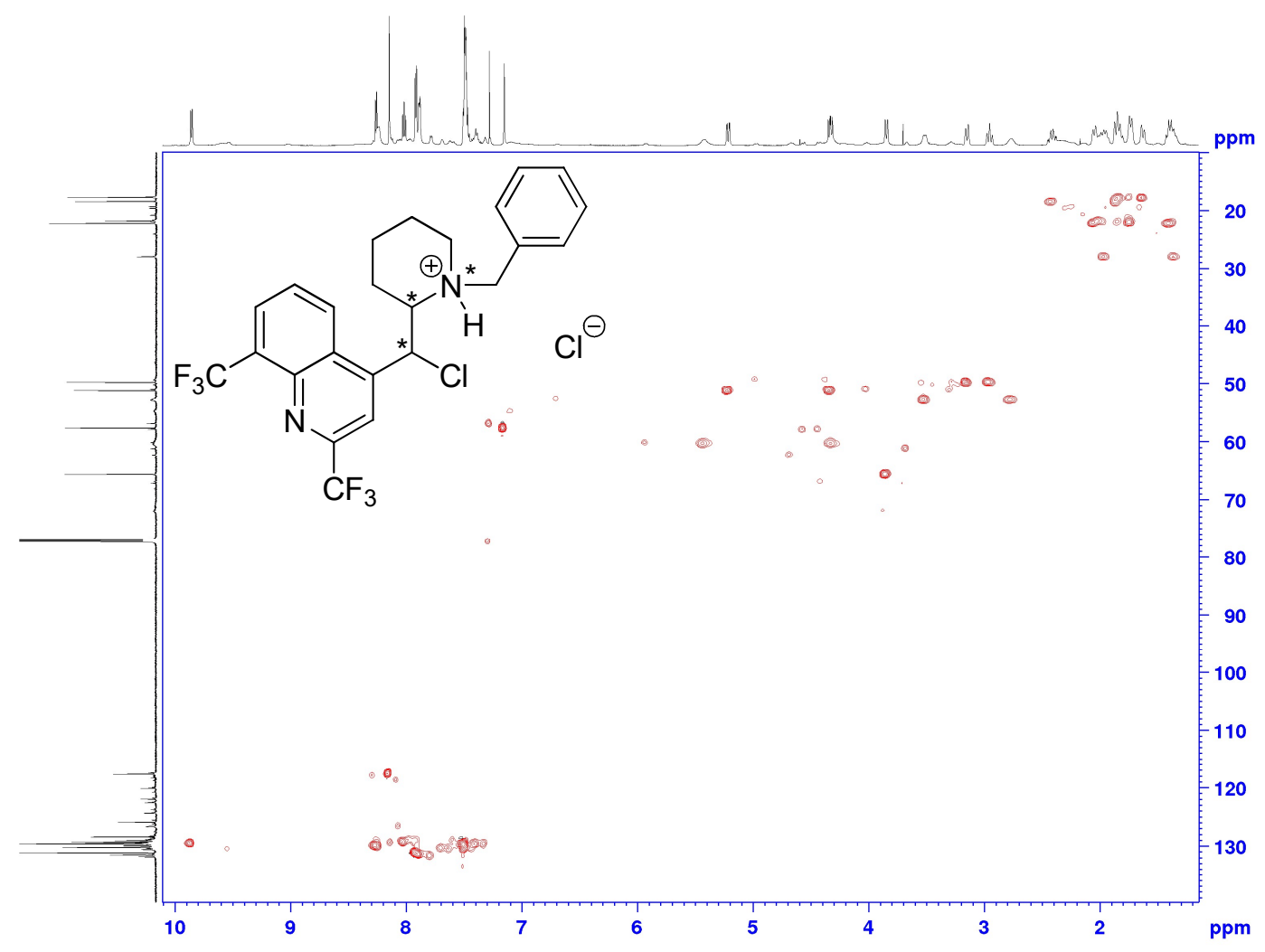

Figure S46. $\quad{ }^{1} \mathrm{H},{ }^{13} \mathrm{C}$ HSQC NMR experiment $\left(600 / 151 \mathrm{MHz}, \mathrm{CDCl}_{3}\right.$, TMS) for 11-chloroderivatives obtained after thionyl chloride treatment of 13-benzyl-mefloquine

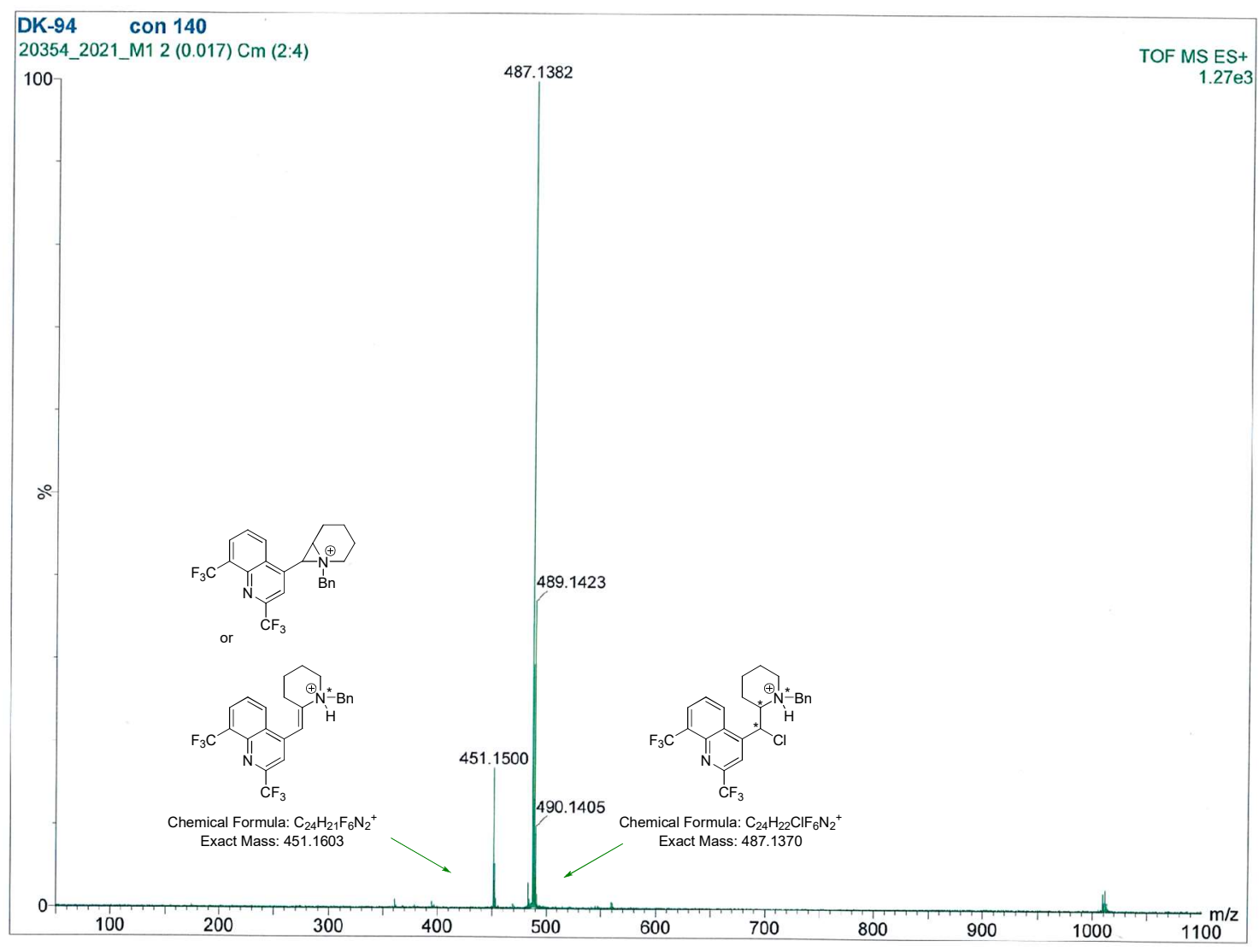

Figure S47. HRMS (ESI-TOF) for 11-chloroderivatives obtained after thionyl chloride treatment of 13-benzyl-mefloquine, plausible structures and their calculated $\mathrm{m} / \mathrm{z}$. 
Chiral HPLC chromatogram plots
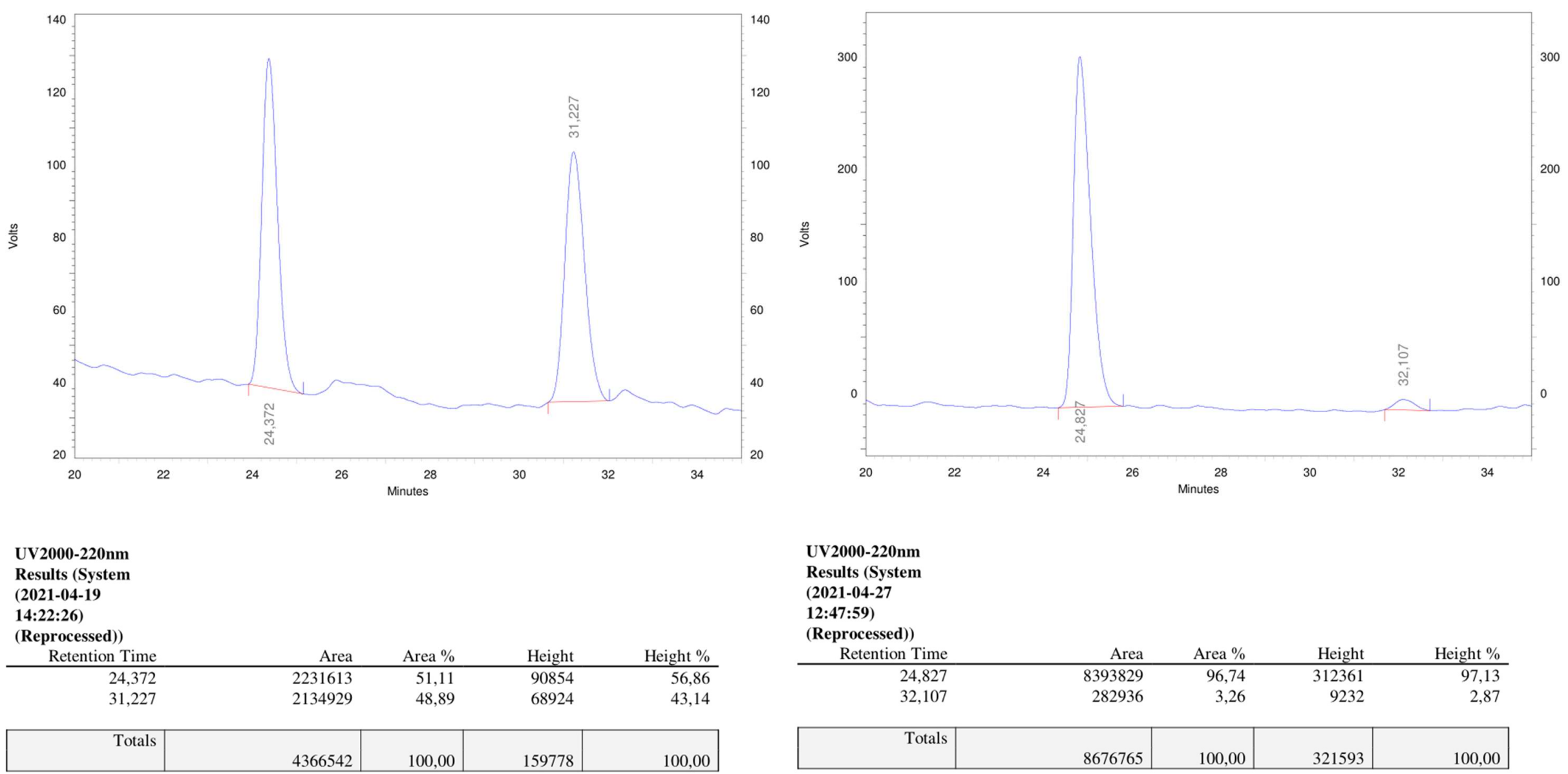

Figure S48. HPLC chromatograms (IC-3 4.6 $\times 250 \mathrm{~mm}$ column, $n$-hexane : 2-propanol, 6:4, flow rate $0.8 \mathrm{~mL} / \mathrm{min}, 220 \mathrm{~nm}$ ) and integration for samples of 3nitromethyl-cyclohexanone obtained with erythro-11-aminomefloquine catalysts: racemic ( \pm )-4 (left) and (+)-4 of over $99 \%$ ee (right). The result of enantiomerically pure catalyst corresponds to Table 1 entry 1. 

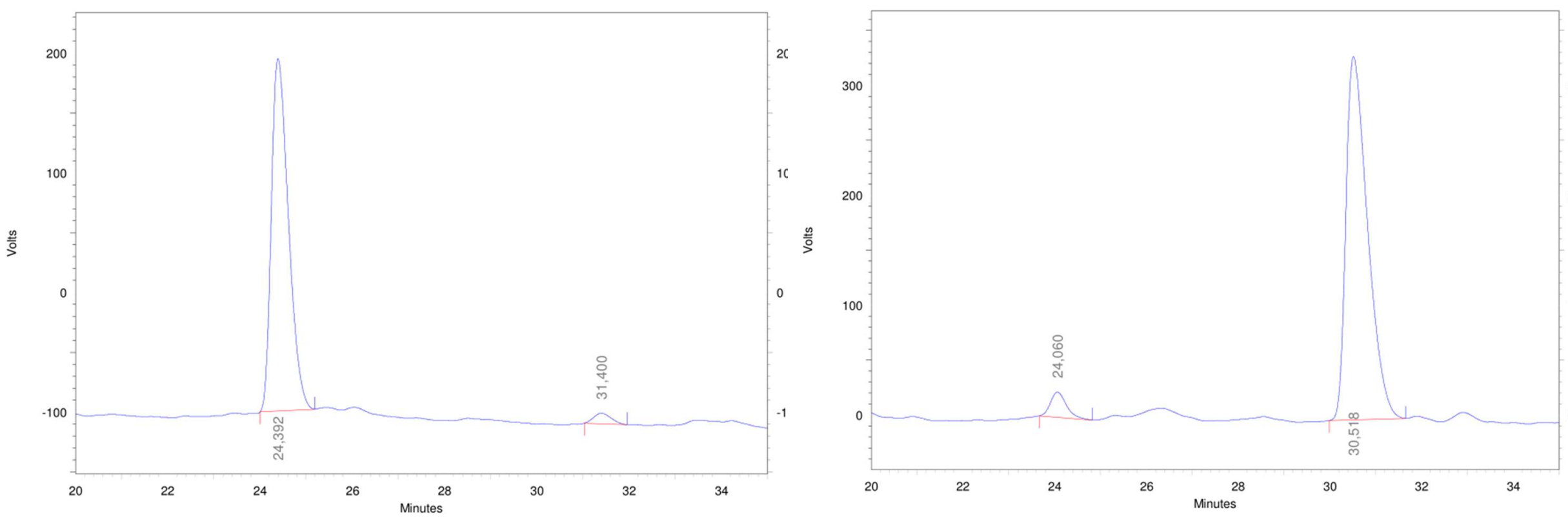

UV2000-220nm

Results (System

(2021-05-12

09:28:29)

(Reprocessed))

\begin{tabular}{rrrrr}
$\begin{array}{r}\text { (Reprocessed)) } \\
\text { Retention Time }\end{array}$ & Area & Area \% & Height & Height \% \\
\hline 24,392 & 7581988 & 97,08 & 294437 & 97,10 \\
31,400 & 228426 & 2,92 & 8785 & 2,90
\end{tabular}

UV2000-220nm

Results (Syste

09:59:40)

(Reprocessed))

\begin{tabular}{rrrrr}
$\begin{array}{r}\text { Retention Time } \\
\text { Rea }\end{array}$ & Area \% & Height & Height \% \\
\hline 30,060 & 549989 & 4,82 & 22845 & 6,46 \\
& 10860726 & 95,18 & 330580 & 93,54 \\
\hline
\end{tabular}

Totals

7810414

100,00

303222

100,00

Totals

11410715

100,00

353425

100,00

Figure S49. HPLC chromatograms (IC-3 4.6 $\times 250 \mathrm{~mm}$ column, hexane : 2-propanol, 6:4, flow rate $0.8 \mathrm{~mL} / \mathrm{min}, 220 \mathrm{~nm}$ ) and integration for sample of 3nitromethyl-cyclohexanone obtained with catalyst (+)-4 of 98\%ee (left) and catalyst (-)-4 of 98\%ee (right). . 


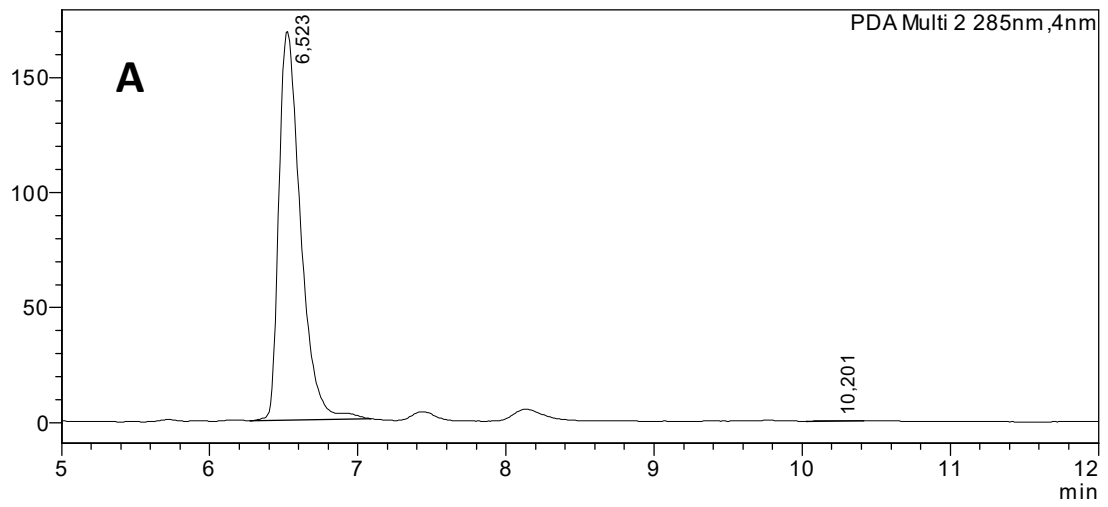

\begin{tabular}{|l|l|l|l|l|l|l|l|}
\hline Peak\# & $\begin{array}{l}\text { Ret. } \\
\text { Time }\end{array}$ & Area & Area\% & Height & Height\% & Resolution(USP) & $\begin{array}{l}\text { Tailing } \\
\text { F. }\end{array}$ \\
\hline 1 & 6,523 & 1714215 & 99,868 & 168744 & 99,856 & -- & 1,565 \\
\hline 2 & 10,201 & 2261 & 0,132 & 243 & 0,144 & 13,672 & 1,058 \\
\hline Total & & 1716476 & 100,000 & 168986 & 100,000 & & \\
\hline
\end{tabular}

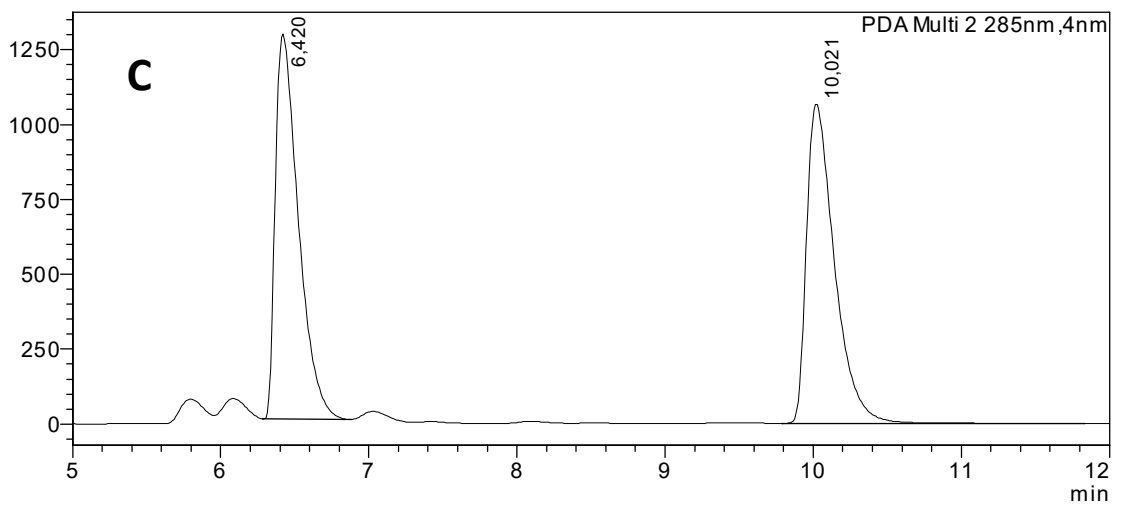

\begin{tabular}{|l|l|l|l|l|l|l|l|}
\hline Peak\# & $\begin{array}{l}\text { Ret. } \\
\text { Time }\end{array}$ & Area & Area\% & Height & Height\% & Resolution(USP) & $\begin{array}{l}\text { Tailing } \\
\text { F. }\end{array}$ \\
\hline 1 & 6,420 & 13995675 & 49,778 & 1285257 & 54,648 & -- & 1,941 \\
\hline 2 & 10,021 & 14120657 & 50,222 & 1066606 & 45,352 & 11,160 & 1,790 \\
\hline Total & & 28116332 & 100,000 & 2351862 & 100,000 & & \\
\hline
\end{tabular}

Figure S50. HPLC chromatograms (IA-3 4.6 $\times 250 \mathrm{~mm}$ column, hexane : 2-propanol, 9:1, flow rate $1 \mathrm{~mL} / \mathrm{min}, 285 \mathrm{~nm}$ ) and integration for samples of $11,13-$ diacetyl derivatives obtained from separated (+)-mefloquine (Panel A), (-)-mefloquine (Panel B) and racemic mefloquine (Panel C).
$\mathrm{mAU}$

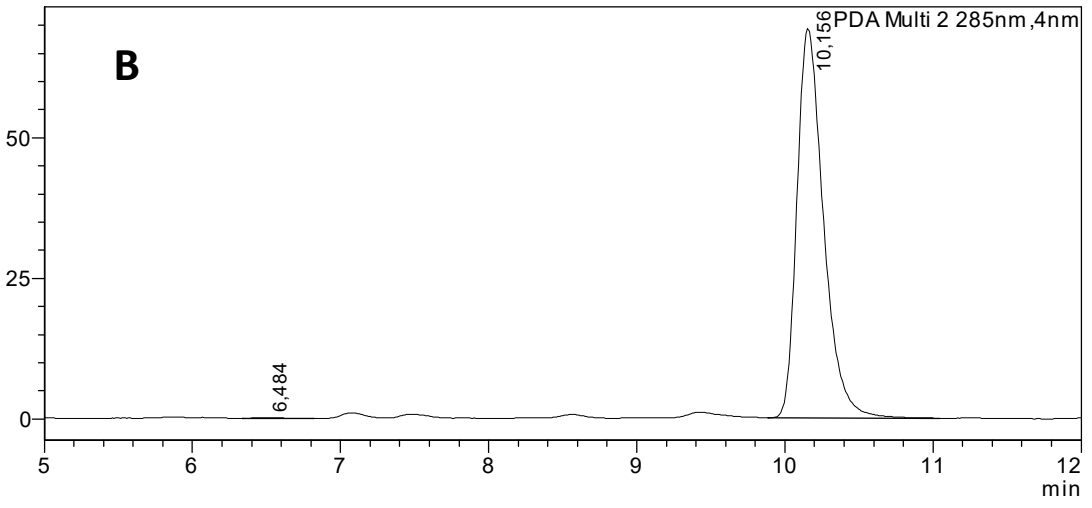

\begin{tabular}{|l|l|l|l|l|l|l|l|}
\hline Peak\# & $\begin{array}{l}\text { Ret. } \\
\text { Time }\end{array}$ & Area & Area\% & Height & Height\% & Resolution(USP) & $\begin{array}{l}\text { Tailing } \\
\text { F. }\end{array}$ \\
\hline 1 & 6,484 & 1206 & 0,139 & 118 & 0,170 & -- & 1,324 \\
\hline 2 & 10,156 & 866406 & 99,861 & 69172 & 99,830 & 11,674 & 1,390 \\
\hline Total & & 867612 & 100,000 & 69290 & 100,000 & & \\
\hline
\end{tabular}


mAU

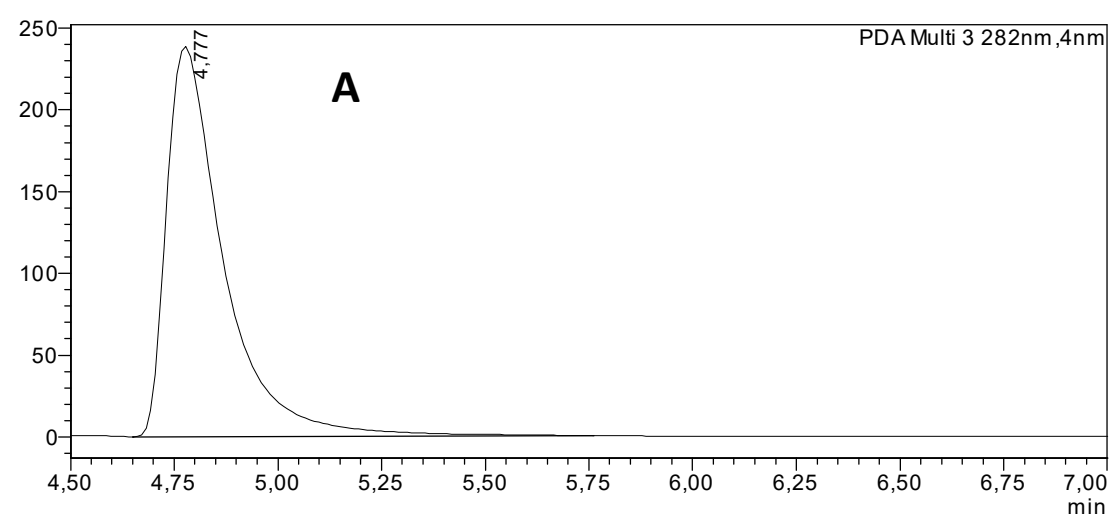

\begin{tabular}{|l|l|l|l|l|l|l|l|}
\hline Peak\# & $\begin{array}{l}\text { Ret. } \\
\text { Time }\end{array}$ & Area & Area\% & Height & Height\% & Resolution(USP) & $\begin{array}{l}\text { Tailing } \\
\text { F. }\end{array}$ \\
\hline 1 & 4,777 & 2279962 & 100,000 & 238368 & 100,000 & -- & 2,115 \\
\hline Total & & 2279962 & 100,000 & 238368 & 100,000 & & \\
\hline
\end{tabular}

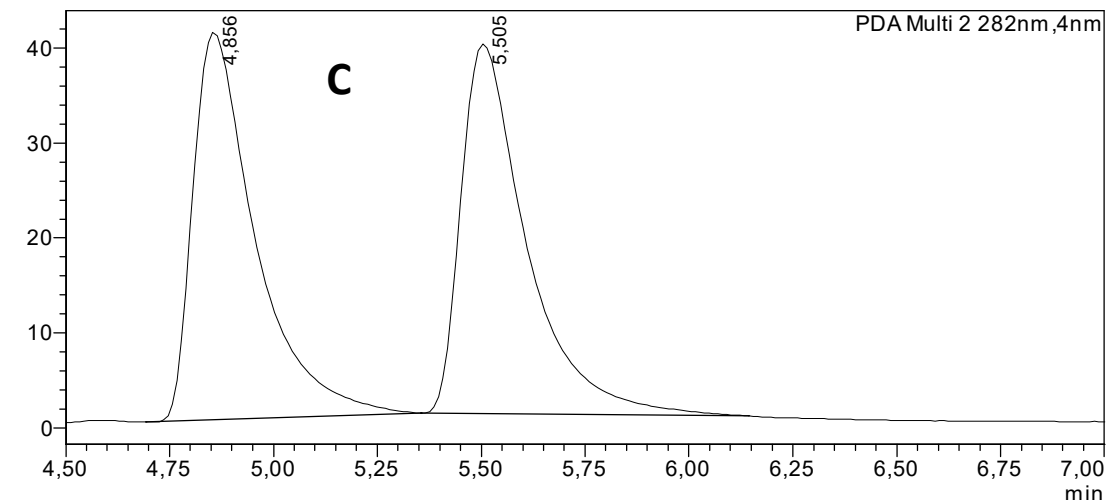

mAU

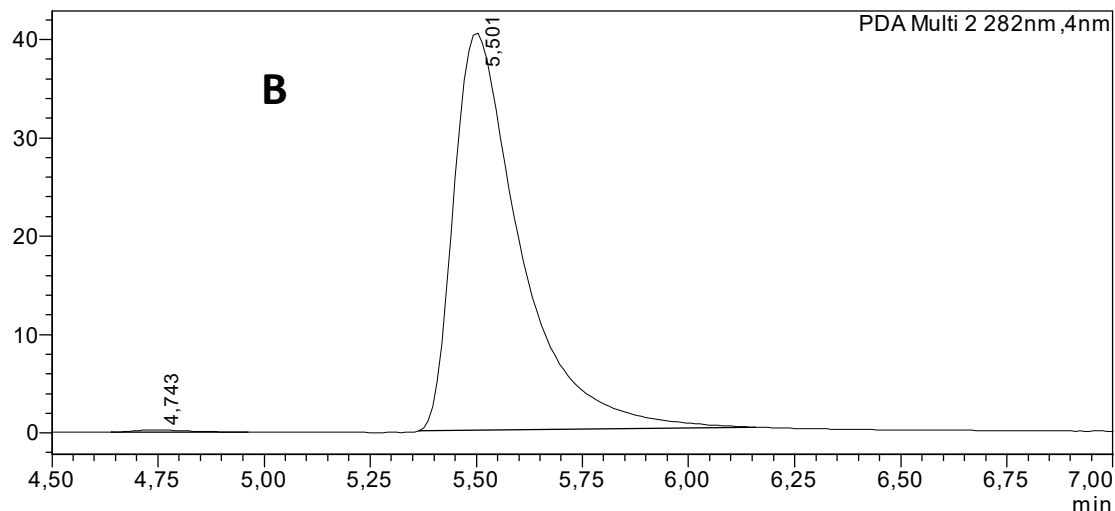

\begin{tabular}{|l|l|l|l|l|l|l|l|}
\hline Peak\# & $\begin{array}{l}\text { Ret. } \\
\text { Time }\end{array}$ & Area & Area\% & Height & Height\% & Resolution(USP) & $\begin{array}{l}\text { Tailing } \\
\text { F. }\end{array}$ \\
\hline 1 & 4,743 & 2292 & 0,496 & 232 & 0,569 & -- & 1,438 \\
\hline 2 & 5,501 & 460129 & 99,504 & 40536 & 99,431 & 2,853 & 2,104 \\
\hline Total & & 462420 & 100,000 & 40768 & 100,000 & & \\
\hline
\end{tabular}

\begin{tabular}{|l|l|l|l|l|l|l|l|}
\hline Peak\# & $\begin{array}{l}\text { Ret. } \\
\text { Time }\end{array}$ & Area & Area\% & Height & Height\% & Resolution(USP) & $\begin{array}{l}\text { Tailing } \\
\text { F. }\end{array}$ \\
\hline 1 & 4,856 & 426983 & 49,970 & 40796 & 51,153 & -- & 2,091 \\
\hline 2 & 5,505 & 427498 & 50,030 & 38957 & 48,847 & 2,351 & 1,985 \\
\hline Total & & 854482 & 100,000 & 79753 & 100,000 & & \\
\hline
\end{tabular}

Figure S51. HPLC chromatograms (IA-3 4.6 $\times 250 \mathrm{~mm}$ column, hexane : 2-propanol, 9:1, flow rate $1 \mathrm{~mL} / \mathrm{min}, 282 \mathrm{~nm})$ and integration for sample of (-)-

$(11 R, 12 S)$-3 obtained from (-)-erythro-mefloquine (Panel A), $(+)-(11 S, 12 R)-\mathbf{3}$ obtained from $(+)$-erythro-mefloquine $($ Panel $\mathbf{B})$, and racemic $\mathbf{3} \cdot \mathrm{HN} 3($ Panel $\mathbf{C})$. 
mAU

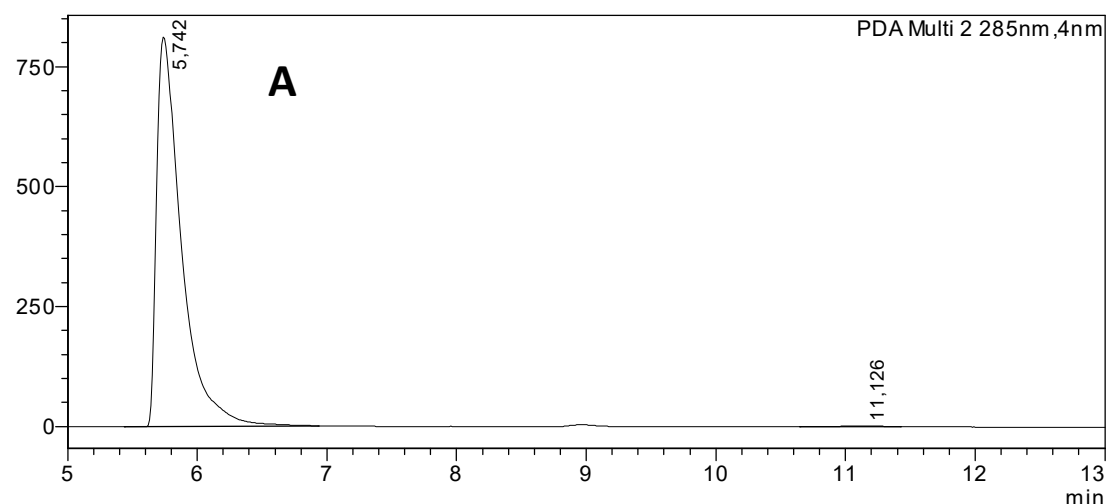

\begin{tabular}{|l|l|l|l|l|l|l|l|}
\hline Peak\# & $\begin{array}{l}\text { Ret. } \\
\text { Time }\end{array}$ & Area & Area\% & Height & Height\% & Resolution(USP) & $\begin{array}{l}\text { Tailing } \\
\text { F. }\end{array}$ \\
\hline 1 & 5,742 & 10763679 & 99,672 & 811421 & 99,811 & -- & 2,598 \\
\hline 2 & 11,126 & 35380 & 0,328 & 1537 & 0,189 & 11,106 & 0,836 \\
\hline Total & & 10799059 & 100,000 & 812958 & 100,000 & & \\
\hline
\end{tabular}

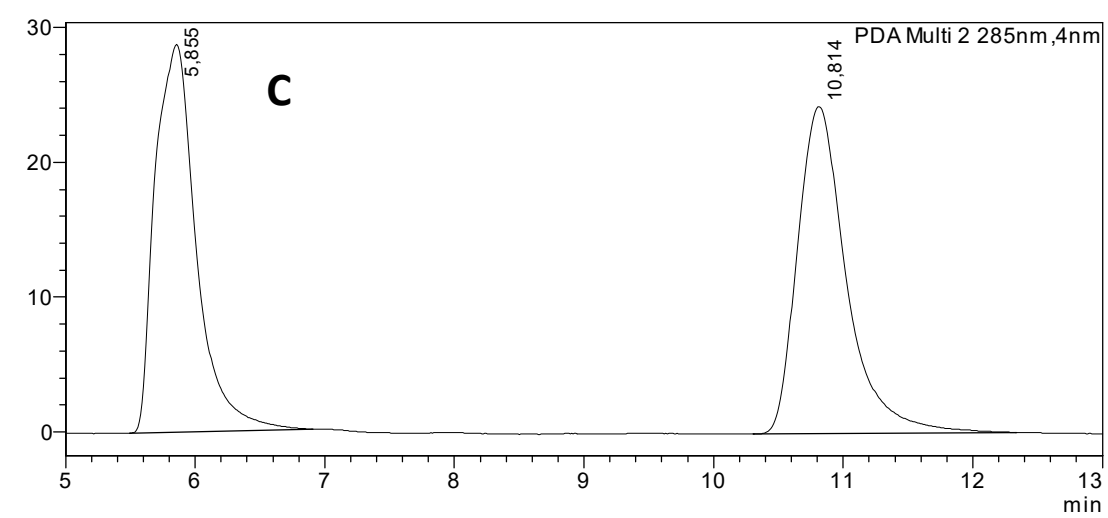

\begin{tabular}{|l|l|l|l|l|l|l|l|}
\hline Peak\# & $\begin{array}{l}\text { Ret. } \\
\text { Time }\end{array}$ & Area & Area\% & Height & Height $\%$ & Resolution(USP) & $\begin{array}{l}\text { Tailing } \\
\text { F. }\end{array}$ \\
\hline 1 & 5,855 & 634497 & 49,915 & 28755 & 54,222 & -- & 1,371 \\
\hline 2 & 10,814 & 636658 & 50,085 & 24277 & 45,778 & 8,375 & 1,452 \\
\hline Total & & 1271155 & 100,000 & 53032 & 100,000 & & \\
\hline
\end{tabular}

Figure S52. HPLC chromatograms (IA-3 4.6 $\times 250 \mathrm{~mm}$ column, hexane : 2-propanol, 9:1, flow rate $1 \mathrm{~mL} / \mathrm{min}, 285 \mathrm{~nm}$ ) and integration for samples of 11,13diacetyl derivatives obtained from $(+)-(11 S, 12 R)$-11-aminomefloquine (Panel A), $(-)-(11 R, 12 S)$-11-aminomefloquine (Panel B), and racemic erythro-11aminomefloquine (Panel C).

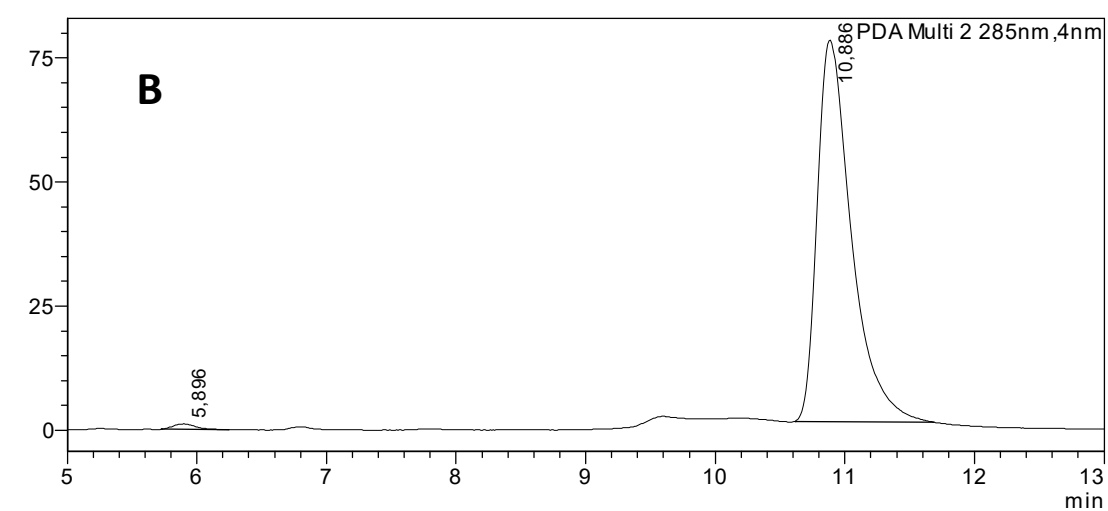

\begin{tabular}{|l|l|l|l|l|l|l|l|}
\hline Peak\# & $\begin{array}{l}\text { Ret. } \\
\text { Time }\end{array}$ & Area & Area\% & Height & Height\% & Resolution(USP) & Tailing F. \\
\hline 1 & 5,896 & 13627 & 0,960 & 1112 & 1,428 & -- & 1,333 \\
\hline 2 & 10,886 & 1406021 & 99,040 & 76786 & 98,572 & 12,516 & 1,673 \\
\hline Total & & 1419647 & 100,000 & 77899 & 100,000 & & \\
\hline
\end{tabular}

mAU 
Scheme for resolution of mefloquine $( \pm-1)$

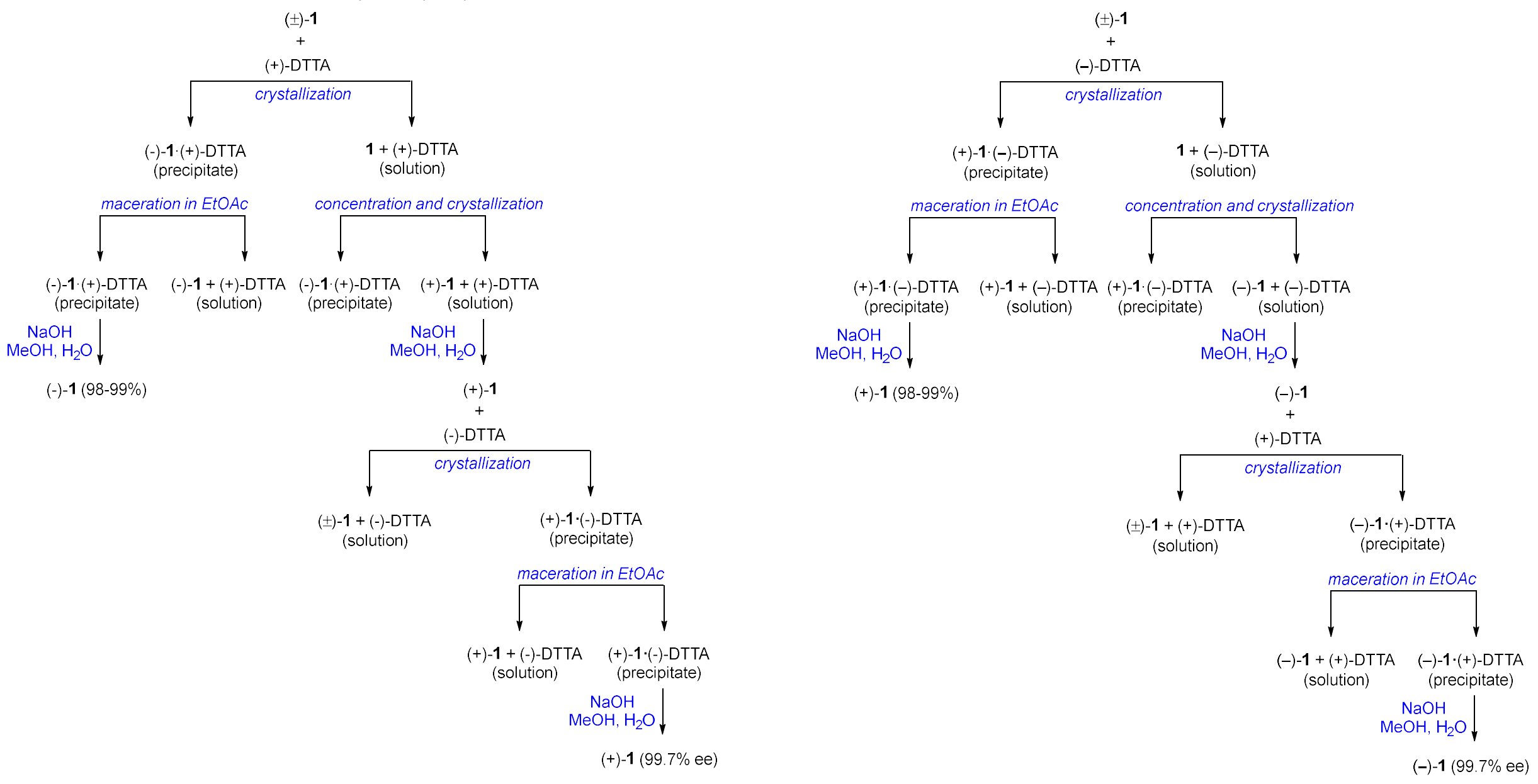

Scheme S1. Resolution scheme of erythro-mefloquine (1) with $O, O$-ditoluyltartaric acids (DTTA) 


\section{Computational data listings for compounds 14 and 15}

Computations performed using Gaussian 16 code $^{\mathrm{S} 1}$

Compound 14 (DFT/B3LYP/CC-PVDZ)

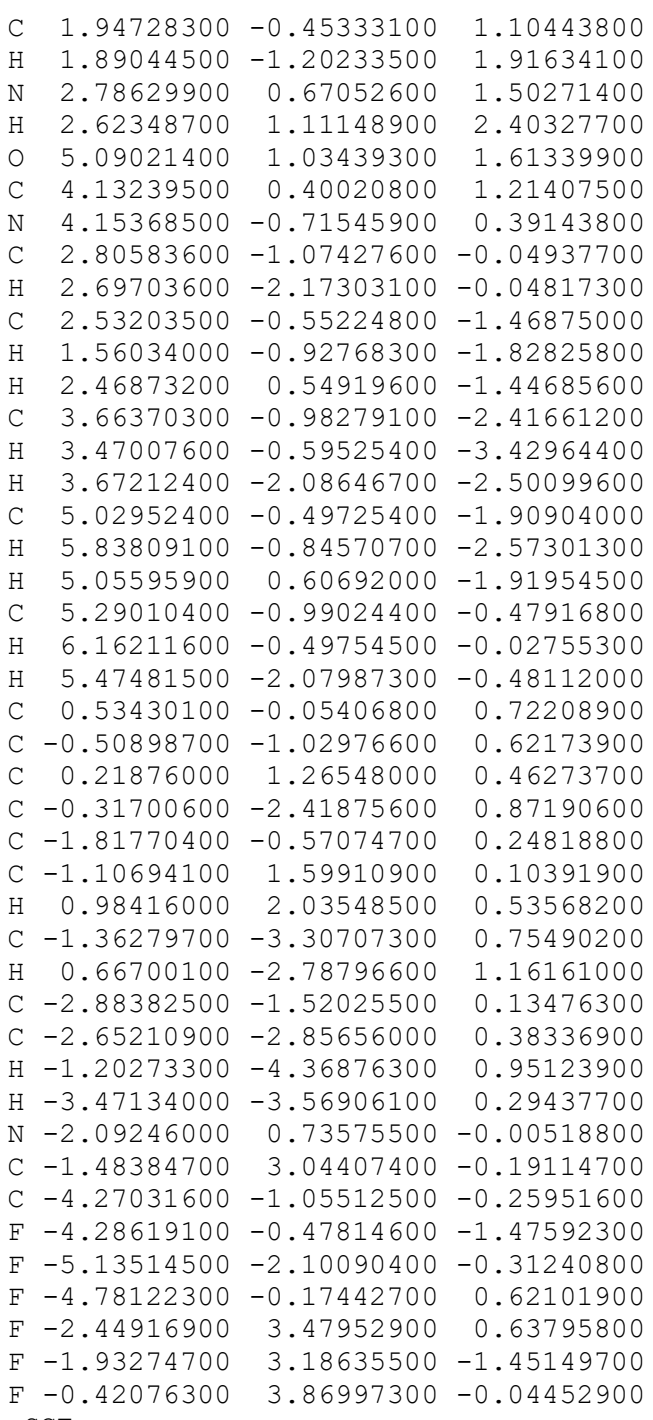

F $-0.42076300 \quad 3.86997300-0.04452$ $\mathrm{SCF}$

Sum of electronic and zero-point Energies=

Sum of electronic and thermal Energies=

Sum of electronic and thermal Enthalpies=

Sum of electronic and thermal Free Energies=

No of imaginary frequencies:

Lowest frequencies:

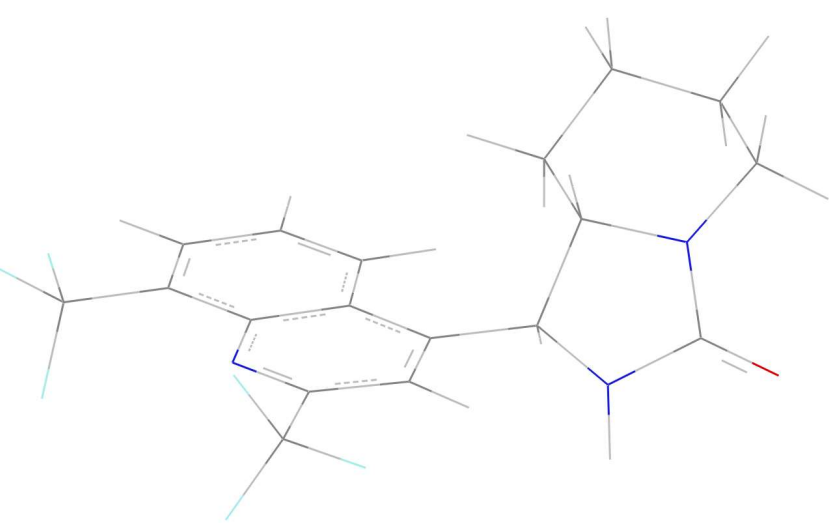

-1533.623191 (Hartree/Particle)

$-1533.305425$

$-1533.282701$

$-1533.281756$

$-1533.360348$

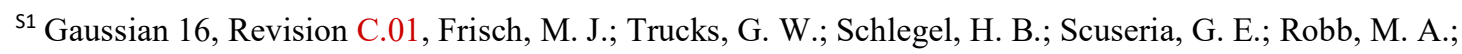

Cheeseman, J. R.; Scalmani, G.; Barone, V.; Petersson, G. A.; Nakatsuji, H.; Li, X.; Caricato, M.; Marenich, A. V.; Bloino, J.; Janesko, B. G.; Gomperts, R.; Mennucci, B.; Hratchian, H. P.; Ortiz, J. V.; Izmaylov, A. F.; Sonnenberg, J. L.; Williams-Young, D.; Ding, F.; Lipparini, F.; Egidi, F.; Goings, J.; Peng, B.; Petrone, A.; Henderson, T.; Ranasinghe, D.; Zakrzewski, V. G.; Gao, J.; Rega, N.; Zheng, G.; Liang, W.; Hada, M.; Ehara, M.; Toyota, K.; Fukuda, R.; Hasegawa, J.; Ishida, M.; Nakajima, T.; Honda, Y.; Kitao, O.; Nakai, H.; Vreven, T.; Throssell, K.; Montgomery, J. A., Jr.; Peralta, J. E.; Ogliaro, F.; Bearpark, M. J.; Heyd, J. J.; Brothers, E. N.; Kudin, K. N.; Staroverov, V. N.; Keith, T. A.; Kobayashi, R.; Normand, J.; Raghavachari, K.; Rendell, A. P.; Burant, J. C.; Iyengar, S. S.; Tomasi, J.; Cossi, M.; Millam, J. M.; Klene, M.; Adamo, C.; Cammi, R.; Ochterski, J. W.; Martin, R. L.; Morokuma, K.; Farkas, O.; Foresman, J. B.; Fox, D. J. Gaussian, Inc., Wallingford CT, 2016. 
Compound 15 (DFT/B3LYP/CC-PVDZ)

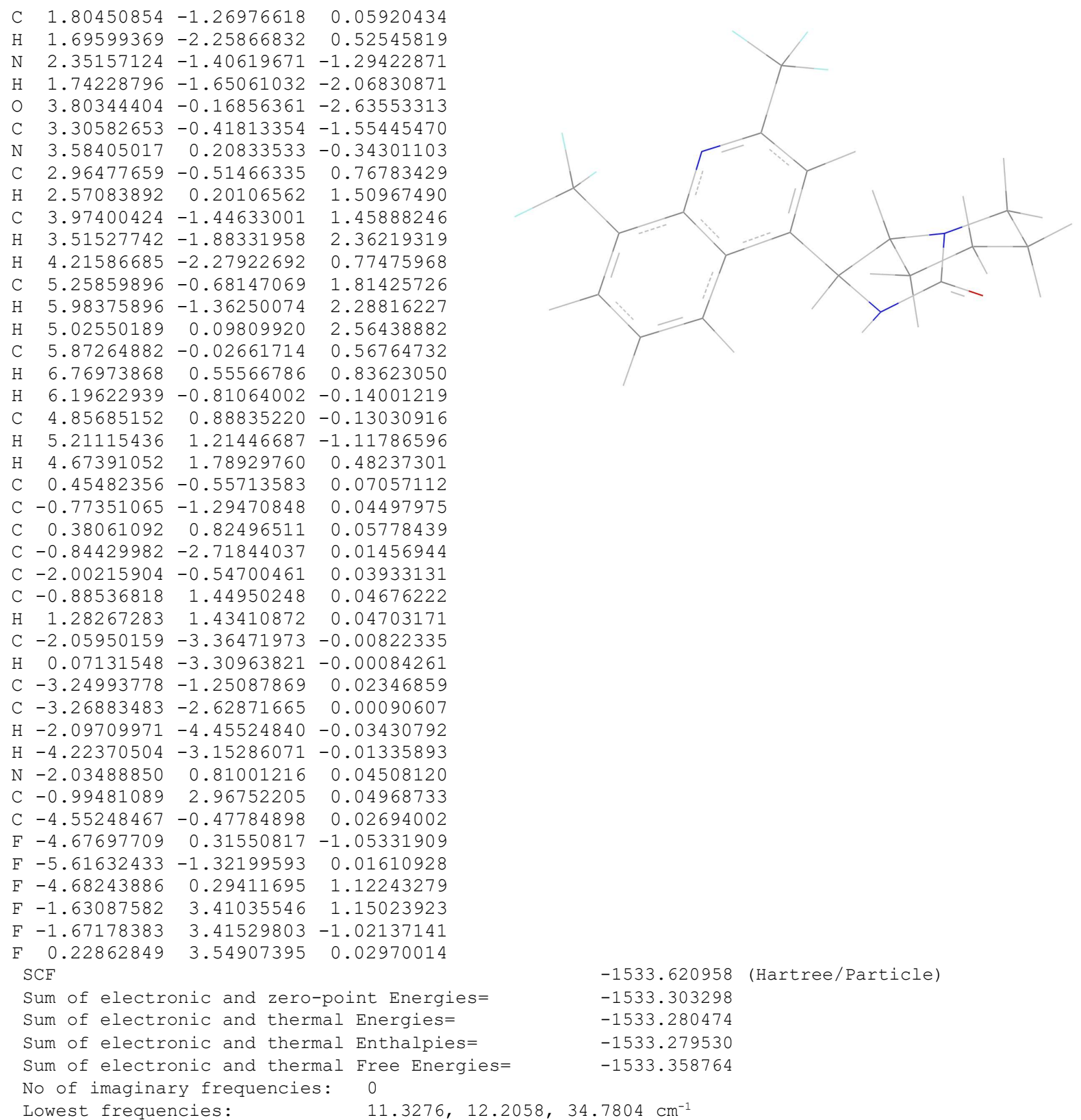

